\title{
Modelos de regressão Birnbaum-Saunders generalizados
}

\section{Michelli Karinne Barros da Silva}

\author{
TESE APRESENTADA \\ $\mathrm{AO}$ \\ INSTITUTO DE MATEMÁTICA E ESTATÍSTICA \\ DA \\ UNIVERSIDADE DE SÃO PAULO \\ PARA OBTENÇÃO DO GRAU DE DOUTOR \\ EM \\ CIÊNCIAS \\ Área de Concentração: Estatística \\ Orientador: Prof. Dr. Gilberto Alvarenga Paula \\ Co-orientador: Prof. Dr. Víctor Leiva Sánchez
}

Durante a realização deste trabalho a autora recebeu apoio financeiro da CAPES e do CNPq

- São Paulo, 11 de junho de 2007 - 


\section{Modelos de regressão Birnbaum-Saunders generalizados}

Este exemplar corresponde à redação final da tese devidamente corrigida e defendida por Michelli Karinne Barros da Silva e aprovada pela comissão julgadora.

São Paulo, 11 de junho de 2007.

Banca examinadora:

- Prof. Dr. Gilberto Alvarenga Paula (Orientador) - IME-USP

- Prof. Dr. Víctor Leiva Sánchez (Co-orientador) - Universidad de Valparaíso - Chile

- Prof. Dr. Edwin Moises Marcos Ortega - ESALQ-USP

- Prof. Dr. Mário de Castro Andrade Filho - ICMC - USP

- Prof. Dr. Francisco Louzada Neto - UFSCar 
Dedico este trabalho a minha filha

Luana e ao meu marido Horácio, por existirem na minha vida. 


\section{Agradecimentos}

A Deus, por não me deixar fraquejar e pela oportunidade de concluir mais uma etapa de minha vida.

Ao professor Gilberto Alvarenga Paula pela oportunidade de ser sua orientanda. Agradeço, a confiança, o incentivo, o apoio, a paciência, a amizade e a excelente orientação durante todo o meu doutorado, em especial, na elaboração deste trabalho.

Ao professor Víctor Leiva Sánchez pela confiança, pelas idéias e sugestões que tanto contribuíram para o desenvolvimento deste trabalho. Agradeço, também, por todo apoio e amizade.

Ao professor Francisco Cribari Neto por ter sido o responsável pela minha vinda à USP.

À professora Sílvia Ferrari pela ótima recepção na chegada a São Paulo.

Ao meu esposo Horácio por seu amor, compreensão, paciência, estímulo e apoio destinados nos momentos mais difíceis.

À minha família que sempre me incentivou, em especial, às minhas "mães" Maria e Elizabeth por todo amor e dedicação.

A todos os amigos e colegas do IME-USP, em especial, Iracema, Kelly, Lourdes, Gisela, Edijane, Clodis e Elier, pela amizade.

Aos amigos Diana e Gustavo, Patrícia e Raydonal, Tatiane e Alessandro, Gilberto e Regele, Jacqueline e Juvêncio, por terem sido mais que amigos, por terem sido a minha família em São Paulo. Obrigada por todo o carinho e incentivo.

Ao amigo Márcio por sua amizade e constante apoio nos momentos difíceis.

Aos amigos Patrícia e Lindomberg, que apesar da distância, sempre me apoiaram.

À Claudia e à Viviana pelo constante incentivo.

A todos os professores e funcionários do IME-USP por suas contribuições à minha formação acadêmica.

Aos professores do Departamento de Estatística da Universidade de Valparaíso-Chile, em especial, ao professor Manuel Galea, pela hospitalidade e pela oportunidade de trabalhar com ele. 
Ao professor Víctor, a sua esposa e toda sua família pela excelente recepção e hospitalidade na minha estadia no Chile.

Aos professores do Departamento de Estatística da Universidade Federal de Pernambuco pelo incentivo.

Aos colegas do Departamento de Matemática e Estatística da Universidade Federal de Campina Grande pelo apoio.

À minha cunhada, Judite, pela recepção na chegada a São Paulo e por todo carinho.

À CAPES e ao CNPq pelo apoio financeiro.

Aos participantes da banca examinadora pelas sugestões.

A todos que direta e indiretamente contribuíram para a realização deste trabalho. 


\section{Resumo}

O modelo de regressão log-Birnbaum-Saunders (log-BS) tem sido largamente aplicado na área de análise de dados de sobrevivência e confiabilidade, particularmente em engenharia, e relaciona o tempo até a ocorrência da falha com algum dano cumulativo que é assumido gaussiano. Numa primeira etapa deste trabalho, consideramos alguns aspectos de diagnóstico de influência e análise de resíduos para este modelo, considerando observações censuradas. Uma vez que é conhecido na literatura que a modelagem sob a suposição de erros normalmente distribuídos pode ser influenciada por observações extremas, mostramos que o mesmo pode ocorrer em modelos cujos erros possuem distribuição log-BS. Numa segunda etapa, desenvolvemos o modelo log-Birnbaum-Saunders generalizado e, particularmente, propomos o modelo $t$ de Student log-BS em que o dano cumulativo é assumido ter distribuição $t$ de Student. Mostramos que as estimativas de máxima verossimilhança do modelo $t$ de Student log-BS são robustas contra observações aberrantes no sentido dos resíduos componente do desvio e tipo martingale. Métodos de diagnóstico de influência e análise de resíduos são desenvolvidos para esse modelo e conjuntos de dados reais são utilizados para ilustrar a teoria desenvolvida. 


\section{Abstract}

The log-Birnbaum-Saunders regression model has been largely applied in survival analysis and reliability to relate the total time until failure with some type of cumulative damage which is assumed to be normally distributed. In the first part of this work, we consider some aspects of influence diagnostics and residual analysis for $\log$-BS regression models with censored data. However, similarly to usual normal regression models, the maximum likelihood estimates from $\log$-BS regression models are also sensitive to outlying observations. Thus, in the second part of this work we consider generalized log-BS regression models, particularly the Student- $t$ logBS model for which the cumulative damage is assumed to follow a Student- $t$ distribution. We show that the maximum likelihood estimates from Student- $t$ log-BS regression models are robust against outlying observations in the sense of the deviance component residual and a martingale-type residual. Influence diagnostic methods are developed for this model and some data sets from the medical area are analyzed under log-BS and Student- $t \log$-BS regression models. 


\section{Sumário}

1 Introdução 1

1.1 Histórico da distribuição Birnbaum-Saunders . . . . . . . . . . . . . . . . 1

1.2 Função de densidade de probabilidade e algumas propriedades . . . . . . . . . 5

1.3 A distribuição Birnbaum-Saunders e a distribuição senh-normal . . . . . . . . 6

1.4 Proposta de trabalho . . . . . . . . . . . . . . . . 7

2 Modelo de Regressão Log-Birnbaum-Saunders 10

2.1 Modelo com resposta log-Birnbaum-Saunders . . . . . . . . . . . . . . . 10

2.2 Influência local . . . . . . . . . . . . . . . . . . . . . . . 12

2.2 .1 Cálculo das curvaturas . . . . . . . . . . . . . . . . . . 15

2.3 Análise de resíduos . . . . . . . . . . . . . . . . . 18

2.3 .1 Aplicações . . . . . . . . . . . . . . . . . . . . . . . . . 21

3 Distribuição Birnbaum-Saunders Generalizada $\quad 37$

3.1 Definição e algumas propriedades da distribuição BSG . . . . . . . . . . . . . . . . 38

3.2 Métodos de estimação . . . . . . . . . . . . . . . . . . . . 49

3.2 .1 Método de máxima verossimilhança . . . . . . . . . . . . . . . . 49

3.2.2 Método dos momentos modificado . . . . . . . . . . . . . . . 51

3.3 Avaliação numérica . . . . . . . . . . . . . . . . . . 53

3.4 Correção de viés por bootstrap . . . . . . . . . . . . . . . . 54

3.4 .1 Avaliação numérica . . . . . . . . . . . . . . . . . 56

4 Modelos de Regressão Log-Birnbaum-Saunders Generalizados $\quad 57$

4.1 Distribuição $\log$-Birnbaum-Saunders generalizada . . . . . . . . . . . . 57

4.2 O modelo de regressão log-Birnbaum-Saunders generalizado . . . . . . . . 58

4.3 Resíduos . . . . . . . . . . . . . . . . . . . . . . . . . . . 62

4.4 Influência local . . . . . . . . . . . . . . . . . . . 63

4.5 Aplicação . . . . . . . . . . . . . . . . . . . 65 
5 Considerações Finais $\quad 70$

5.1 Trabalhos futuros . . . . . . . . . . . . . . . . . . . . 71

$\begin{array}{ll}\text { Apêndice } & 71\end{array}$

$\begin{array}{ll}\text { A Expressões do Capítulo 2 } & 72\end{array}$

A.1 Funções escore. . . . . . . . . . . . . . . . . . . . . . . . . . 72

A.2 Matriz hessiana . . . . . . . . . . . . . . . . . . 73

A.3 Cálculo das curvaturas . . . . . . . . . . . . . . . . . . 76

A.4 Resíduo componente do desvio . . . . . . . . . . . . . . . . . . . . . 79

B $\quad$ Tabelas referentes às simulações do Capítulo $3 \quad 81$

$\begin{array}{ll}\text { Referências } & 89\end{array}$ 


\section{Lista de Figuras}

1.1 funções densidade da distribuição Birnbaum-Saunders para os valores de $\alpha$ indicados e $\beta=1 \ldots \ldots \ldots \ldots \ldots \ldots \ldots$

1.2 funções de risco da distribuição Birnbaum-Saunders para os valores de $\alpha$ indi-

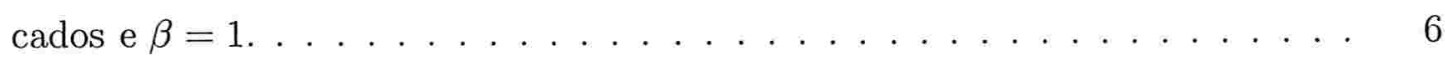

1.3 funções densidade da distribuição senh-normal com parâmetros $\gamma=0, \sigma=1$

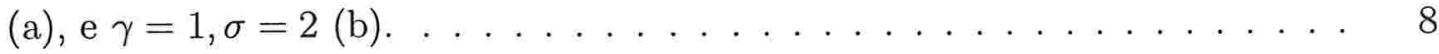

2.1 gráficos normais de probabilidade para os resíduos componente do desvio padronizado com porcentagem de censura de $10 \%$. . . . . . . . . . . . . . . 22

2.2 gráficos normais de probabilidade para os resíduos componente do desvio padronizado com porcentagem de censura de $30 \%$. . . . . . . . . . . . . . . . 23

2.3 gráficos normais de probabilidade para os resíduos componente do desvio padronizado com porcentagem de censura de $50 \%$. . . . . . . . . . . . . . 24

2.4 gráficos dos resíduos $r_{D C_{i}}^{*}$ (a) e $r_{M D_{i}}^{*}$ (b) contra os valores ajustados. . . . . . 26

2.5 gráfico da medida de alavanca generalizada contra o índice das observações. . . 26

2.6 gráficos normais de probabilidade para os resíduos $r_{D C_{i}}^{*}$ (a) e $r_{M D_{i}}^{*}$ (b) com envelopes. . . . . . . . . . . . . . . . . . . . . . 27

2.7 gráficos de $\left|l_{\max }\right|$ contra o índice das observações sob o esquema de perturbação da ponderação de casos: (a) interesse em $\beta$, (b) interesse em $\alpha . \ldots . . .27$

2.8 gráfico de $\left|l_{\max }\right|$ para $\boldsymbol{\theta}$ contra o índice das observações sob o esquema de perturbação da variável resposta. . . . . . . . . . . . . . . . . . . . . . 28

2.9 gráficos de $\left|l_{\max }\right|$ contra o índice das observações sob o esquema de perturbação da variável explicativa $x_{2}$ : (a) interesse em $\boldsymbol{\beta}$, (b) interesse em $\alpha . \quad \ldots . .28$

2.10 gráficos de $\left|l_{\max }\right|$ contra o índice das observações sob o esquema de perturbação da ponderação de casos não censurados: (a) interesse em $\beta$, (b) interesse em $\alpha$.

2.11 gráficos de $\left|l_{\max }\right|$ contra o índice das observações sob o esquema de perturbação da ponderação de casos censurados: (a) interesse em $\beta$, (b) interesse em $\alpha$. . . 


\section{Lista de Tabelas}

2.1 estimativas dos parâmetros e os níveis descritivos $(p)$ para o conjunto indicado. 31

2.2 mudanças relativas ( $\mathrm{RC}$, em \%) e os correspondentes níveis descritivos entre parênteses. . . . . . . . . . . . . . . . . . . . . . 32

2.3 mudanças relativas (-RC- em \%) e os correspondentes níveis descritivos entre parênteses. . . . . . . . . . . . . . . . . . . . . 36

3.1 exemplos de distribuições na classe Birnbaum-Saunders generalizada. . . . . . 39

$3.2 \mathbb{E}\left(U^{2 n}\right)$ em que $U \sim E C(0,1 ; g)$, com $n=1,2,3,4$, para as distribuições elípticas indicadas. . . . . . . . . . . . . . . . . . . 46

4.1 expressões para $W_{g}(u)$ e $W_{g}^{\prime}(u)$ para algumas distribuições elípticas $\ldots \ldots \ldots$. . 61

4.2 mudanças relativas ( $\mathrm{RC}$, em \%) e os correspondentes níveis descritivos entre parênteses. . . . . . . . . . . . . . . . . . . . . .

B.1 EMV, EMM e ECB para os parâmetros $\alpha$ e $\beta$ considerando a distribuição BSG gerada pela distribuição $t$ de Student $\operatorname{com} \nu=5 \ldots \ldots$. . . . . . . 82

B.2 EMV, EMM e ECB para os parâmetros $\alpha$ e $\beta$ considerando a distribuição BSG gerada pela distribuição $t$ de Student com $\nu=7 \ldots \ldots$. . . . . . . . . 83

B.3 EMV, EMM e ECB para os parâmetros $\alpha$ e $\beta$ considerando a distribuição BSG gerada pela distribuição Pearson VII com $q=4$ e $r=6 \ldots \ldots \ldots$. . . . 84

B.4 EMV, EMM e ECB para os parâmetros $\alpha$ e $\beta$ considerando a distribuição BSG gerada pela distribuição Pearson VII com $q=10$ e $r=20 \ldots \ldots$. . . . . . 85

B.5 erro quadrático médio das estimativas dos parâmetros $\alpha$ e $\beta$ considerando a distribuição BSG gerada pela distribuição $t$ de Student $\operatorname{com} \nu=5$. . . . . 86

B.6 erro quadrático médio das estimativas dos parâmetros $\alpha$ e $\beta$ considerando a distribuição BSG gerada pela distribuição $t$ de Student $\operatorname{com} \nu=7$. . . . . . 87

B.7 erro quadrático médio das estimativas dos parâmetros $\alpha$ e $\beta$ considerando a distribuição BSG gerada pela distribuição Pearson VII com $q=4$ e $r=6$. . 88 
B.8 erro quadrático médio das estimativas dos parâmetros $\alpha$ e $\beta$ considerando a distribuição BSG gerada pela distribuição Pearson VII com $q=10$ e $r=20$. 


\section{Capítulo 1}

\section{Introdução}

\subsection{Histórico da distribuição Birnbaum-Saunders}

A fadiga é um dano estrutural que ocorre quando um material é exposto a flutuações de estresse e tensão. Modelos estatísticos para processos de fadiga nos possibilitam descrever a variação aleatória dos tempos de falha associados aos materiais expostos à fadiga como um resultado de diferentes padrões e forças cíclicas. Esses materiais podem ser caracterizados por parâmetros de distribuições de vida. Tais caracterizações são importantes para predizer a performance dos materiais sob diferentes condições (veja, por exemplo, Galea, Leiva e Paula, 2004). Entre os modelos probabilísticos que têm sido popularmente propostos para descrever a vida por fadiga encontram-se as distribuições gama, gaussiana inversa, lognormal e Weibull, que se ajustam com grande precisão na região central da distribuição de vida. Todavia, o interesse geralmente está nos percentis mais baixos ou mais altos da distribuição, região em que costuma haver poucos dados, o que conduz a um pobre ajuste dos modelos acima mencionados. Birnbaum e Saunders (1969a), motivados por problemas de vibração, encontrados em aviões comerciais novos e de falhas de materiais, usaram o conhecimento sobre um tipo particular de fadiga para derivar uma nova família de distribuições, a qual modela o tempo de vida de materiais e equipamentos sujeitos a cargas dinâmicas. A seguir descreveremos brevemente como esta nova família foi derivada.

Birnbaum e Saunders (1969a) fizeram as seguintes suposições sobre o processo de fadiga:

1. Um material é sujeito a um padrão cíclico de tensão e força;

2. A falha do material ocorre devido ao desenvolvimento e ao crescimento de uma fissura dominante dentro do material, ou seja, quando o tamanho da fissura excede certo nível de resistência, denotado por $\omega$; 
3. A seqüência de tensão imposta ao material é a mesma de ciclo para ciclo;

4. A extensão incremental da fissura $X_{i}$ resultante da aplicação da $i$-ésima oscilação de carga é uma variável aleatória com uma distribuição que só depende da fissura atual causada pela tensão neste ciclo;

5. A extensão da fissura durante o $(j+1)$-ésimo ciclo é

$$
Y_{j+1}=X_{j m+1}+\cdots+X_{j m+m}, \text { para } j=0,1, \ldots,
$$

em que $X_{j m+i}$ é a extensão da fissura (possivelmente microscópica) após a $i$-ésima oscilação de carga do $(j+1)$-ésimo ciclo;

6. As extensões das fissuras em diferentes ciclos são independentes;

7. A extensão total da fissura, $Y_{j}$, devido ao $j$-ésimo ciclo é uma variável aleatória que segue uma distribuição com média $\mu$ e variância $\sigma^{2}, \forall j=1,2, \ldots$.

Assim, a extensão total da fissura após $n$ ciclos é dada pela variável aleatória

$$
W_{n}=\sum_{j=1}^{n} Y_{j}
$$

com função de distribuição acumulada (fda) dada por

$$
H_{n}(\omega)=P\left(W_{n} \leq \omega\right),
$$

para $n=1,2, \ldots$

Seja $N$ o número de ciclos requeridos até que seja observada a falha. A função de distribuição da variável aleatória $N$ é

$$
P(N \leq n)=P\left(\sum_{j=1}^{n} Y_{j}>\omega\right)=P\left(W_{n}>\omega\right)=1-H_{n}(\omega) .
$$

Agora, supondo que os $Y_{j}$ 's são variavéis aleatórias independentes e identicamente distribuídas segue do teorema central do limite que

$$
\begin{aligned}
P(N \leq n) & =P\left(\sum_{j=1}^{n} \frac{Y_{j}-\mu}{\sigma \sqrt{n}}>\frac{\omega-n \mu}{\sigma \sqrt{n}}\right) \\
& =1-P\left(\sum_{j=1}^{n} \frac{Y_{j}-\mu}{\sigma \sqrt{n}} \leq \frac{\omega-n \mu}{\sigma \sqrt{n}}\right)
\end{aligned}
$$




$$
\begin{aligned}
& \cong 1-\Phi\left(\frac{\omega-n \mu}{\sigma \sqrt{n}}\right)=1-\Phi\left(-\frac{n \mu-\omega}{\sigma \sqrt{n}}\right) \\
& =\Phi\left(\frac{\mu \sqrt{n}}{\sigma}-\frac{\omega}{\sigma \sqrt{n}}\right)
\end{aligned}
$$

em que $\Phi(\cdot)$ representa a fda da distribuição normal padrão.

Birnbaum e Saunders (1969a) usaram a equação (1.2) para definir uma distribuição contínua de vida. De acordo com os autores, se $n$ é substituído por uma variável aleatória real não-negativa $t$, então a variável aleatória $T$ é a extensão contínua da variável aleatória discreta $N$. Desta forma, $T$ representa o tempo total até que ocorra falha. Assim, tomando

$$
\alpha=\frac{\sigma}{\sqrt{\mu \omega}} \quad \text { e } \quad \beta=\frac{\omega}{\mu},
$$

a função de distribuição acumulada (fda) de $T$ é escrita como

$$
F_{T}(t)=P(T \leq t)=\Phi\left[\frac{1}{\alpha}\left(\sqrt{\frac{t}{\beta}}-\sqrt{\frac{\beta}{t}}\right)\right], \quad t>0,
$$

em que $\alpha>0$ e $\beta>0$. Dizemos, então, que $T$ segue uma distribuição Birnbaum-Saunders com parâmetros $\alpha$ e $\beta$ e denotamos por $T \sim B S(\alpha, \beta)$.

Portanto, segue que $T \sim B S(\alpha, \beta)$ é definida em termos da distribuição normal mediante a variável aleatória

$$
T=\beta\left[\frac{\alpha Z}{2}+\sqrt{\left(\frac{\alpha Z}{2}\right)^{2}+1}\right]^{2}
$$

em que $Z \sim N(0,1)$.

A distribuição Birnbaum-Saunders (BS) vem recebendo ultimamente um grande destaque na literatura. A seguir apresentaremos alguns dos principais trabalhos envolvendo essa distribuição. Birnbaum e Saunders (1969b) obtêm originalmente os estimadores de máxima verossimilhança para $\alpha$ e $\beta$. Engelhardt, Bain e Wright (1981) propuseram intervalos de confiança e testes de hipóteses para os parâmetros da distribuição considerando um deles como parâmetro de perturbação desconhecido, ou seja, obtiveram intervalos de confiança para $\alpha$ considerando $\beta$ como parâmetro de perturbação desconhecido e, para $\beta$ considerando $\alpha$ como parâmetro de perturbação desconhecido. Desmond (1985) consolidou a justificação física usada para construção desta distribuição, relaxando algumas suposições feitas por Birnbaum e Saunders (1969a) e apresentou uma derivação mais geral baseada em um modelo biológico. Desmond (1986) estabeleceu uma conexão entre a distribuição gaussiana inversa e a distribuição BS. Rieck e Nedelman (1991) propuseram um modelo log-linear para a distribuição 
BS e desenvolveram métodos de estimação. Achcar e Espinosa (1991) desenvolveram métodos bayesianos em testes de vida acelerados considerando um modelo log-linear para a distribuição BS proposto por Rieck e Nedelman (1991). Lu e Chang (1997) utilizaram métodos bootstrap para construir intervalos de predição. Dupuis e Mills (1998) propuseram métodos robustos de estimação dos parâmetros da distribuição BS. Rieck (1999) derivou a função geradora de momentos para a distribuição seno hiperbólico normal (senh-normal) que pode ser usada para obter momentos de ordem inteira ou fracionária da distribuição BS. McCarter (1999) discutiu a estimação dos parâmetros da distribuição BS para o caso de amostras censuradas do tipo II.

Algumas generalizações e extensões da distribuição BS foram propostas na última década. Owen e Padgett (1999) desenvolveram a distribuição BS com três parâmetros. Recentemente, Díaz-García e Leiva $(2005,2006)$ propuseram uma nova classe de distribuições de vida generalizando a distribuição BS a partir de distribuições de contornos elípticos. Segundo os autores, esta generalização baseia-se, além do aspecto elíptico, na busca de distribuições de vida que cresçam mais rapidamente e que possuam caudas com menor ou maior curtose do que a distribuição BS, entre outras propriedades tais como: distribuições de vida que não possuem momentos e que sejam bimodais. É válido salientar que, neste caso, o uso de distribuições elípticas para gerar a distribuição Birnbaum-Saunders generalizada (BSG) não segue uma justificação física, como no caso clássico (distribuição BS baseada na distribuição normal), mas razões puramente estatísticas e/ou matemáticas. Além disso, a distribuição BS é um caso particular da distribuição BSG e muitas das propriedades da versão clássica são transferidas para a versão generalizada. Mais recentemente, Vilca-Labra e Leiva (2006), baseados em argumentos semelhantes aos empregados por Díaz-García e Leiva (2005, 2006), obtiveram uma maior generalização ao desenvolver a distribuição BS mediante distribuições elípticas assimétricas. Assim, a distribuição Birnbaum-Saunders duplamente generalizada (BSG"), como eles denominaram, é mais flexível na curtose e assimetria do que todas as versões anteriores da distribuição BS.

Modelagem estatística sob distribuição BSG não tem recebido muita atenção. No caso clássico, alguns trabalhos têm sido desenvolvidos, os quais podem ser vistos em Rieck e Nedelman (1991), Owen e Padgett (1999, 2000) e Tsionas (2001). Em Galea, Leiva e Paula (2004) aspectos relacionados a diagnóstico de influência em modelos de regressão log-BirnbaumSaunders (log-BS) com dados não censurados foram estudados. 


\subsection{Função de densidade de probabilidade e algumas propriedades}

A função densidade de probabilidade (fdp) de $T$ é obtida a partir de (1.3) e é dada por

$$
f_{T}(t)=\frac{1}{\sqrt{2 \pi}} \exp \left[-\frac{1}{2 \alpha^{2}}\left(\frac{t}{\beta}+\frac{\beta}{t}-2\right)\right] \frac{t^{-3 / 2}(t+\beta)}{2 \alpha \sqrt{\beta}}
$$

em que $t, \alpha, \beta>0$.

O parâmetro $\alpha$ controla a forma da distribuição. A distribuição BS torna-se assimétrica à medida que $\alpha$ cresce, e simétrica, em torno de $\beta$, à medida que $\alpha$ se aproxima de zero. Este comportamento pode ser visto na Figura 1.1. O parâmetro $\beta$ é o parâmetro de escala e também a mediana da distribuição, ou seja, $F_{T}(\beta)=\Phi(0)=1 / 2$.

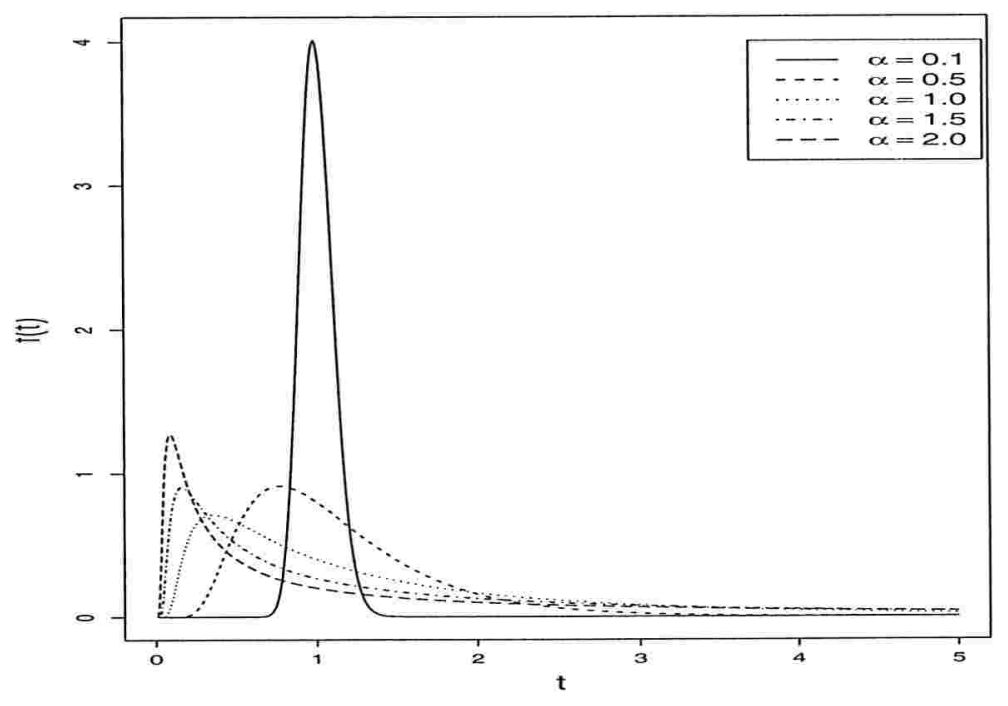

Figura 1.1: funções densidade da distribuição Birnbaum-Saunders para os valores de $\alpha$ indicados e $\beta=1$.

A média e a variância da distribuição existem e são dadas, respectivamente, por

$$
\mathbb{E}(T)=\beta\left(1+\frac{\alpha^{2}}{2}\right) \quad \text { e } \quad \operatorname{Var}(T)=(\alpha \beta)^{2}\left(1+\frac{5}{4} \alpha^{2}\right) .
$$

Se $c>0$ tem-se que $c T \sim B S(\alpha, c \beta)$. A distribuição BS também possui a propriedade de ser fechada sob reciprocidade, ou seja, $T^{-1} \sim B S\left(\alpha, \beta^{-1}\right)$. A função de sobrevivência e a função de risco da distribuição BS são dadas, respectivamente, por 


$$
S_{T}(t)=1-F_{T}(t)=1-\Phi\left[\frac{1}{\alpha}\left(\sqrt{\frac{t}{\beta}}-\sqrt{\frac{\beta}{t}}\right)\right]
$$

$\mathrm{e}$

$$
h_{T}(t)=\frac{f_{T}(t)}{S_{T}(t)}
$$

em que $f_{T}(t)$ e $S_{T}(t)$ são dados em (1.5) e (1.7), respectivamente.

A função de risco $h_{T}(t)$ assume valor zero em $t=0$, cresce até um valor máximo e depois decresce até uma constante positiva, como podemos observar na Figura 1.2.

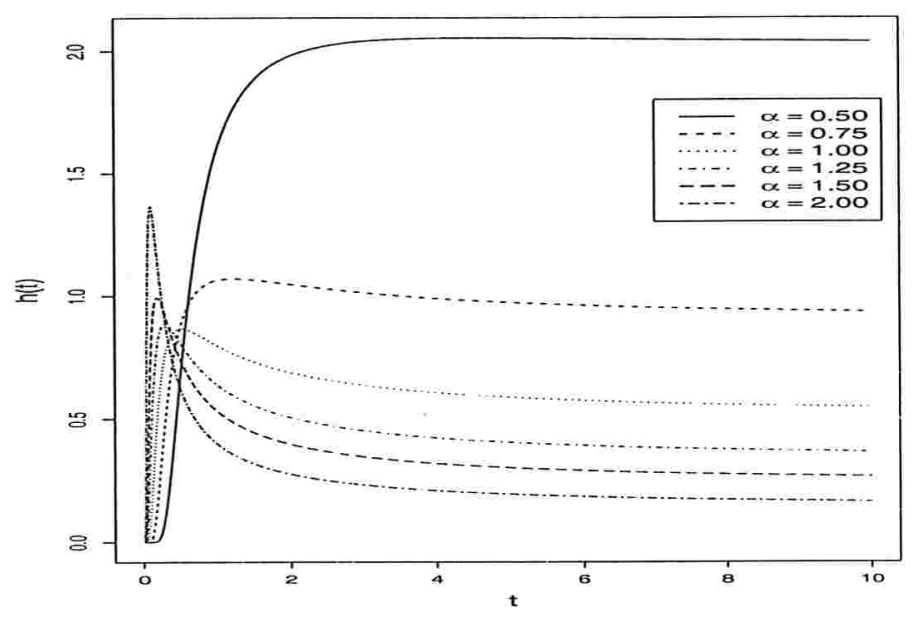

Figura 1.2: funções de risco da distribuição Birnbaum-Saunders para os valores de $\alpha$ indicados e $\beta=1$.

\subsection{A distribuição Birnbaum-Saunders e a distribuição senh-normal}

A distribuição senh-normal (SN), desenvolvida por Rieck e Nedelman (1991), também é definida através de uma transformação da distribuição normal por meio da variável aleatória $Y=\operatorname{arcsenh}(\alpha Z / 2) \sigma+\gamma$, em que $Z \sim N(0,1), \alpha>0$ é o parâmetro de forma, $\gamma \in \mathbb{R}$ é o parâmetro de locação e $\sigma>0$ é o parâmetro de escala. Denotamos $Y \sim S N(\alpha, \gamma, \sigma)$. A função densidade de probabilidade de $Y$ é dada por 


$$
f_{Y}(y)=\left(\frac{2}{\alpha \sigma \sqrt{2 \pi}}\right) \cosh \left(\frac{y-\gamma}{\sigma}\right) \exp \left[-2 \alpha^{-2} \operatorname{senh}^{2}\left(\frac{y-\gamma}{\sigma}\right)\right], y \in \mathbb{R}
$$

Temos ainda que se $Y \sim S N(\alpha, \gamma, \sigma)$, então $Z=2 \alpha^{-1} \operatorname{senh}((Y-\gamma) / \sigma) \sim N(0,1)$. Segue então que a fda de $Y$ é

$$
F_{Y}(y)=\Phi\left[\frac{2}{\alpha} \operatorname{senh}\left(\frac{y-\gamma}{\sigma}\right)\right]
$$

em que $\Phi(\cdot)$ é a fda da distribuição normal padrão.

Rieck (1989) mostrou que a distribuição senh-normal é simétrica em torno de $\gamma$, unimodal para $\alpha \leq 2$ e bimodal para $\alpha>2$. Além disso, se $Y \sim S N(\alpha, \gamma, \sigma)$, então $Z=2(Y-\gamma) / \sigma \alpha$ converge em distribuição para a distribuição normal padrão quando $\alpha \rightarrow 0$. A média e a variância da distribuição SN podem ser obtidas usando a função geradora de momentos dada por

$$
m(s)=\exp (\mu s)\left[\frac{K_{a}\left(\delta^{-2}\right)+K_{b}\left(\delta^{-2}\right)}{2 K_{\frac{1}{2}}\left(\delta^{-2}\right)}\right],
$$

em que $a=(\sigma s+1) / 2, b=(\sigma s-1) / 2$ e $K(\cdot)$ é a função de Bessel modificada do terceiro tipo, dada por $K_{\lambda}(w)=(1 / 2)(w / 2)^{\lambda} \int_{0}^{\infty} y^{-\lambda-1} e^{-y-\left(w^{2} / 4 y\right)} d y$ (ver Gradshteyn e Randzhik, 2000, p. 907).

Rieck e Nedelman (1991) provaram que se $T \sim B S(\alpha, \beta)$, então $Y=\log T \sim S N(\alpha, \gamma, \sigma=$ 2), em que $\gamma=\log \beta$. Por esta razão, a distribuição senh-normal é também denominada distribuição log-Birnbaum-Saunders.

Observa-se que se $Y \sim S N(\alpha, \gamma, \sigma)$, quando $\alpha$ cresce, a curtose da distribuição SN também cresce. Em particular, para $\alpha \leq 2$, temos unimodalidade e curtose menor do que o caso normal. Entretanto, para $\alpha>2$, à medida que $\alpha$ cresce, a distribuição SN começa a apresentar bimodalidade, possui modas que são mais separadas e curtose maior do que o caso normal. O parâmetro $\gamma$ modifica a locação e o parâmetro $\sigma$ a escala da distribuição, como podemos acompanhar na Figura 1.3.

\subsection{Proposta de trabalho}

O principal objetivo deste trabalho é o estudo dos modelos de regressão log-linear Birnbaum-Saunders generalizados (log-BSG). Inicialmente, no Capítulo 2, estenderemos os resultados de diagnóstico de influência desenvolvïdos por Galea, Leiva e Paula (2004) para observações censuradas sob modelos de regressão linear log-Birnbaum-Saunders (log-BS). Derivamos as 

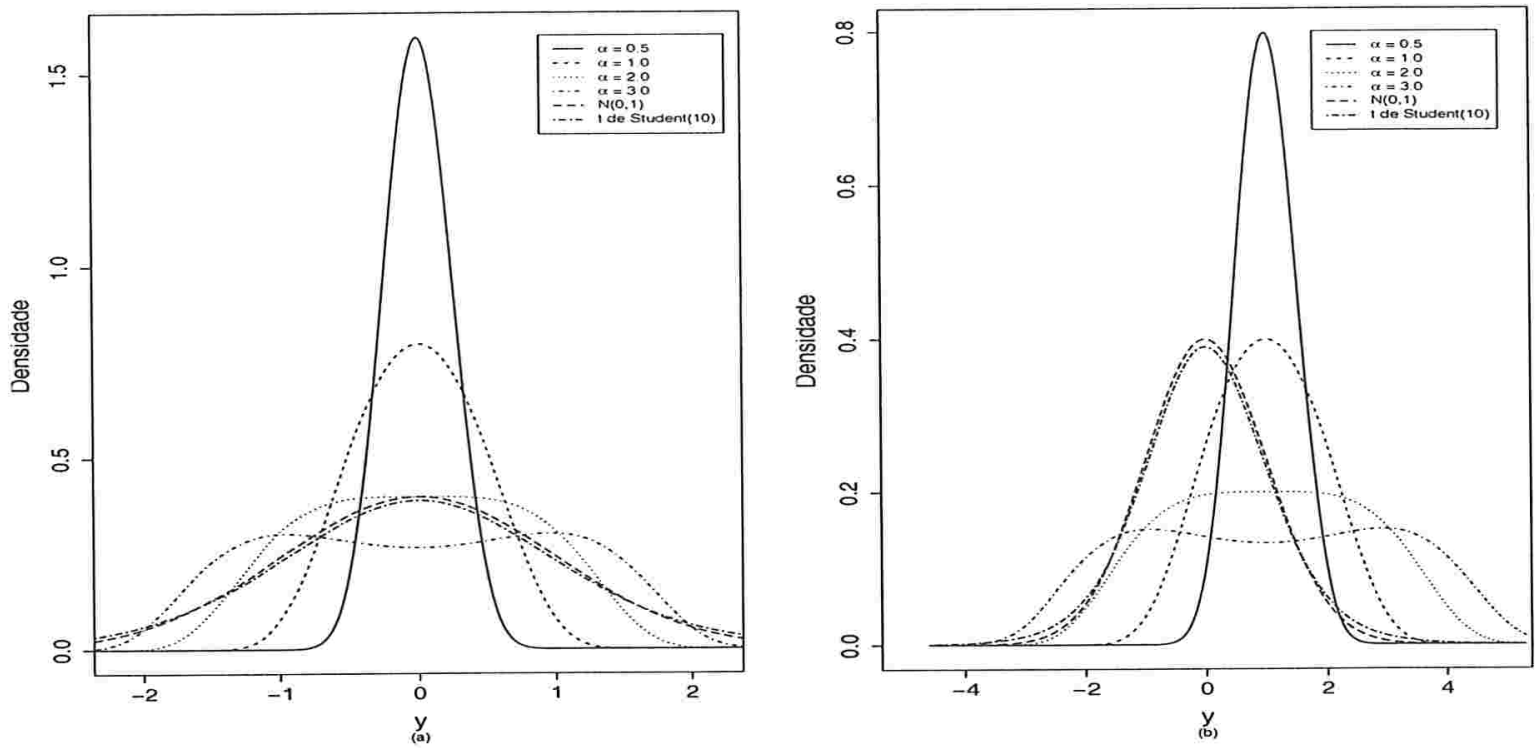

Figura 1.3: funções densidade da distribuição senh-normal com parâmetros $\gamma=0, \sigma=1$ (a), e $\gamma=1, \sigma=2(\mathrm{~b})$.

curvaturas normais da influência local sob vários esquemas de perturbação. Adicionalmente, propomos dois resíduos (resíduo componente do desvio e resíduo tipo martingale), para avaliar possíveis afastamentos da suposição de que os erros seguem distribuição log-BS, bem como para detectar observações extremas com alguma influência desproporcional nos resultados do ajuste. Apresentamos também um estudo de simulação de Monte Carlo objetivando a análise do comportamento da distribuição empírica dos resíduos propostos. Aplicações com dados reais da área médica que não tinham sido analisados sob esta perspectiva de diagnóstico são apresentadas. No Capítulo 3, apresentaremos, inicialmente, a distribuição Birnbaum-Saunders generalizada (BSG) proposta por Díaz-García e Leiva (2005, 2006). Em seguida, descreveremos os momentos dessa distribuição e apresentaremos alguns aspectos não discutidos em Díaz-García e Leiva (2005, 2006). Discutiremos também a estimação dos parâmetros da distribuição utilizando os métodos de máxima verossimilhança e dos momentos modificados, este último proposto por Ng, Kundu e Balakrishnan (2003), assim como resultados de simulações referentes ao processo de estimação. Apresentaremos também uma proposta de correção de viés através do método bootstrap. No Capítulo 4, desenvolveremos a classe de modelos de regressão log-BSG, que generaliza os modelos log-BS bem conhecidos e aplicados à análise de dados de sobrevivência. Daremos uma atenção especial ao modelo $t$ de Student log-BS, onde mostraremos que as estimativas de máxima verossimilhança são menos sensíveis a observações 
atípicas, pois são atribuídos pesos menores a essas observações no processo de estimação. Apresentaremos alguns resultados inferenciais, derivaremos as curvaturas normais da influência local sob vários esquemas de perturbação e apresentaremos alguns estudos com os resíduos componente do desvio e tipo martingale. Por fim, analisaremos novamente o conjunto de dados apresentado no Capítulo 2. O Capítulo 5, por sua vez, apresenta as conclusões e alguns direcionamentos para trabalhos futuros. 


\section{Capítulo 2}

\section{Modelo de Regressão}

\section{Log-Birnbaum-Saunders}

Neste capítulo, discutiremos a aplicação do método de influência local (Cook, 1986) em modelos de regressão log-lineares com resposta Birnbaum-Saunders e observações censuradas. Esses modelos, como foi mencionado no Capítulo 1, são comumente utilizados para modelar a propagação de um dano acumulado até a ocorrência de um processo de falha. Como medida de influência, consideramos o afastamento pela verossimilhança (likelihood displacement) sob três esquemas de perturbação: ponderação de casos, perturbação da variável resposta e perturbação da variável explicativa. Derivamos as matrizes apropriadas para obter a influência local nos parâmetros estimados do modelo e realizamos uma análise residual baseada nos resíduos componente do desvio e tipo martingale. Apresentamos também um estudo de simulação de Monte Carlo a fim de investigar o comportamento da distribuição empírica dos resíduos propostos. Aplicações com dados reais são apresentadas.

\subsection{Modelo com resposta log-Birnbaum-Saunders}

Consideramos o modelo de regressão linear log-Birnbaum-Saunders

$$
y_{i}=\mathbf{x}_{i}^{\top} \boldsymbol{\beta}+\varepsilon_{i}, \quad i=1, \ldots, n,
$$

em que $y_{i}$ é o logaritmo do tempo de sobrevivência ou do tempo de censura para a $i$-ésima unidade experimental, $\boldsymbol{\beta}=\left(\beta_{1}, \beta_{2}, \ldots, \beta_{p}\right)^{\top}$ é o vetor de parâmetros desconhecidos a ser estimado, $\mathbf{x}_{i}=\left(x_{i 1}, x_{i 2}, \ldots, x_{i p},\right)^{\top}$ são observações de $p$ variáveis explicativas e $\varepsilon_{i}$ são variáveis aleatórias independentes e identicamente distribuídas tais que $\varepsilon_{i} \sim S N(\alpha, 0,2)$. Supomos 
censura não informativa e que os tempos de sobrevivência e de censura são independentes. Denotaremos por $D$ e $C$ o conjunto de indivíduos para o qual $y_{i}$ é o logaritmo do tempo de sobrevivência ou o logaritmo do tempo censurado, respectivamente. O logaritmo da função de verossimilhança total do modelo (2.1) para $\theta=\left(\alpha, \beta^{\top}\right)^{\top}$ possui a seguinte forma:

$$
l(\boldsymbol{\theta}) \propto \sum_{i \in D} l_{i}(\boldsymbol{\theta})+\sum_{i \in C} l_{i c}(\boldsymbol{\theta})
$$

em que $l_{i}(\theta)=\log f_{Y}\left(y_{i} ; \boldsymbol{\theta}\right), l_{i c}(\boldsymbol{\theta})=\log S_{Y}\left(y_{i} ; \boldsymbol{\theta}\right)$ e $S_{Y}(y ; \boldsymbol{\theta})=1-F_{Y}(y ; \boldsymbol{\theta})$ é a função de sobrevivência, com $f_{Y}(\cdot ; \boldsymbol{\theta})$ dado em $(1.8)$ e $F_{Y}(\cdot ; \theta)$ em (1.9).

Assim, o logaritmo da função de verossimilhança total para $\boldsymbol{\theta}$ pode ser escrito como

$$
l(\boldsymbol{\theta}) \propto \sum_{i \in D}\left\{\log \left(\xi_{i 1}\right)-\frac{1}{2}\left[\log (8 \pi)+\xi_{i 2}^{2}\right]\right\}+\sum_{i \in C} \log \left[1-\Phi\left(\xi_{i 2}\right)\right],
$$

em que $\xi_{i 1}=\frac{2}{\alpha} \cosh \left(\frac{y_{i}-\mu_{i}}{2}\right), \xi_{i 2}=\frac{2}{\alpha} \operatorname{senh}\left(\frac{y_{i}-\mu_{i}}{2}\right)$ e $\mu_{i}=\mathrm{x}_{i}^{\top} \boldsymbol{\beta}$, para $i=1,2, \ldots, n$.

As funções escore para $\alpha$ e $\beta_{j}(j=1, \ldots, p)$ são dadas, respectivamente, por

$$
U_{\alpha}(\boldsymbol{\theta})=\frac{\partial l(\boldsymbol{\theta})}{\partial \alpha}=\frac{1}{\alpha}\left[\sum_{i \in D}\left(\xi_{i 2}^{2}-1\right)+\sum_{i \in C} \xi_{i 2} h\left(\xi_{i 2}\right)\right]
$$

e

$$
U_{\beta_{j}}(\boldsymbol{\theta})=\frac{\partial l(\boldsymbol{\theta})}{\partial \beta_{j}}=\sum_{i \in D}\left[\frac{x_{i j}}{\alpha^{2}} \operatorname{senh}\left(y_{i}-\mu_{i}\right)-\frac{x_{i j}}{2} \tanh \left(\frac{y_{i}-\mu_{i}}{2}\right)\right]+\frac{1}{2} \sum_{i \in C} x_{i j} \xi_{i 1} h\left(\xi_{i 2}\right),
$$

em que $h\left(\xi_{i 2}\right)=\frac{\phi\left(\xi_{i 2}\right)}{1-\Phi\left(\xi_{i 2}\right)}$, com $\phi(\cdot)$ e $\Phi(\cdot)$ denotando a fdp e fda da distribuição normal padrão, respectivamente.

Os estimadores de máxima verossimilhança dos coeficientes de regressão e do parâmetro de forma são soluções das equações $U_{\beta_{j}}(\theta)=0(j=1, \ldots, p)$ e $U_{\alpha}(\boldsymbol{\theta})=0$. Porém, neste caso, as equações não apresentam soluções analíticas, sendo necessária a utilização de métodos iterativos para a obtenção das raízes. Essas podem ser obtidas numericamente, maximizando o logaritmo da função de verossimilhança e usando um algoritmo de otimização não-linear, por exemplo, Newton-Raphson ou um algoritmo quase-Newton (BFGS, por exemplo). Usamos o método BFGS (ver, por exemplo, Press et al., 1992) com derivadas analíticas. Como valores iniciais consideramos para o parâmetro de forma $\alpha$ a estimativa sob o modelo de regressão linear log-BS para observações não-censuradas (ver detalhes em Galea, Leiva e Paula, 2004) e para o vetor $\boldsymbol{\beta}$ as estimativas de mínimos quadrados.

A inferência assintótica para o vetor de parâmetros $\theta=\left(\alpha, \beta^{\top}\right)^{\top}$ pode ser baseada na normalidade assintótica do estimador de máxima verossimilhança $\widehat{\theta}$, dada por

$$
\widehat{\boldsymbol{\theta}} \dot{\sim} N_{(p+1)}\left(\theta, \Sigma_{\theta}\right),
$$


em que $\Sigma_{\theta}$ é a matriz de variâncias e covariâncias de $\widehat{\boldsymbol{\theta}}$, que pode ser aproximada pela matriz de informação observada $-\ddot{\boldsymbol{L}}_{\theta \theta}^{-1}$, avaliada em $\widehat{\boldsymbol{\theta}}$, obtida a partir de

$$
\ddot{\boldsymbol{L}}_{\theta \theta}=\left[\begin{array}{cc}
\ddot{\boldsymbol{L}}_{\alpha \alpha} & \ddot{\boldsymbol{L}}_{\alpha \beta} \\
\ddot{\boldsymbol{L}}_{\beta \alpha} & \ddot{\boldsymbol{L}}_{\beta \beta}
\end{array}\right]=\left[\begin{array}{cc}
\operatorname{tr}(\boldsymbol{G}) & \boldsymbol{K}^{\top} \boldsymbol{X} \\
\boldsymbol{X}^{\top} \boldsymbol{K} & \boldsymbol{X}^{\top} \boldsymbol{V} \boldsymbol{X}
\end{array}\right],
$$

em que $\boldsymbol{G}=\operatorname{diag}\left\{g_{1}(\boldsymbol{\theta}), \ldots, g_{n}(\boldsymbol{\theta})\right\}$, com

$$
g_{i}(\theta)=\left\{\begin{array}{l}
\frac{1}{\alpha^{2}}-\frac{3 \xi_{i 2}^{2}}{\alpha^{2}}, \text { se } i \in D \\
-\frac{2}{\alpha^{2}} \xi_{i 2} h\left(\xi_{i 2}\right)-\frac{1}{\alpha^{2}} \xi_{i 2}^{2} h^{\prime}\left(\xi_{i 2}\right), \text { se } i \in C ;
\end{array}\right.
$$

$\boldsymbol{K}=\left(k_{1}(\boldsymbol{\theta}), \ldots, k_{n}(\boldsymbol{\theta})\right)^{\top}, \mathrm{com}$

$$
k_{i}(\boldsymbol{\theta})=\left\{\begin{array}{l}
-\frac{2}{\alpha^{3}} \operatorname{senh}\left(y_{i}-\mu_{i}\right), \text { se } i \in D \\
-\frac{1}{2 \alpha} \xi_{i 1} h\left(\xi_{i 2}\right)-\frac{1}{\alpha^{3}} \operatorname{senh}\left(y_{i}-\mu_{i}\right) h^{\prime}\left(\xi_{i 2}\right), \text { se } i \in C
\end{array}\right.
$$

e $\boldsymbol{V}=\operatorname{diag}\left\{v_{1}(\boldsymbol{\theta}), \ldots, v_{n}(\boldsymbol{\theta})\right\}, \mathrm{com}$

$$
v_{i}(\theta)=\left\{\begin{array}{l}
\frac{1}{4} \operatorname{sech}^{2}\left(\frac{y_{i}-\mu_{i}}{2}\right)-\frac{1}{\alpha^{2}} \cosh \left(y_{i}-\mu_{i}\right), \text { se } i \in D \\
-\frac{1}{4} \xi_{i 2} h\left(\xi_{i 2}\right)-\frac{1}{4} \xi_{i 1}^{2} h^{\prime}\left(\xi_{i 2}\right), \text { se } i \in C
\end{array}\right.
$$

em que $h(\cdot)$ é dado em (2.4) e $h^{\prime}(\cdot)$ é a sua derivada, e $\xi_{i 1}$ e $\xi_{i 2}$ são dados em (2.2).

No Apêndice A.2 encontram-se cálculos mais detalhados para a obtenção da matriz $\ddot{\boldsymbol{L}}_{\theta \theta}$ dada em (2.5).

\subsection{Influência local}

Uma maneira eficaz para detectar observações influentes é a análise de diagnóstico. A deleção de pontos é talvez a técnica mais conhecida para avaliar o impacto da retirada de uma observação, ou de um conjunto de observações, nas estimativas dos parâmetros da regressão. Tal técnica foi introduzida por Cook (1977) em modelos normais lineares e depois foi adaptada para diversas classes de modelos. Para o caso normal linear, veja, por exemplo, Belsley, Kuh e Welsch (1980), Cook e Weisberg (1982), Atkinson (1985) e Chatterjee e Hadi (1988). Alguns exemplos de extensões dessa metodologia são: Moolgavkar, Lustbader e Venzon (1984), que estendem para modelos de regressão não-linear com aplicações em estudos emparelhados; Paula e Peres (1988), que discutem a deleção de pontos em modelos lineares generalizados com parâmetros restritos na forma $\mathbf{C} \boldsymbol{\beta} \geq 0$; Davison e Tsai (1992) e Cordeiro e Paula (1992), que fazem uma extensão para modelos cuja distribuição não pertence à família exponencial 
de distribuições; Galea, Riquelme e Paula (2000), que analisam esta técnica em modelos de contornos elípticos multivariados. Uma desvantagem que pode ocorrer com a deleção individual de pontos é que essa metodologia pode deixar de detectar pontos conjuntamente influentes. Entretanto, Cook (1986) propõe o método de influência local que é uma importante ferramenta na análise da influência conjunta das observações nos resultados de um ajuste de regressão. O método de influência local tem como objetivo principal avaliar mudanças nos resultados da análise quando pequenas perturbações são incorporadas ao modelo e/ou aos dados. Se essas perturbações causarem efeitos desproporcionais, pode ser indício de que o modelo está mal ajustado ou que possam existir afastamentos sérios das suposições feitas para o mesmo. Vários autores têm aplicado a metodologia de influência local em modelos de regressão mais geral do que o modelo de regressão normal linear (veja, por exemplo, Paula, 1993; Galea, Paula e Bolfarine, 1997; Galea, Riquelme e Paula, 2000 e Díaz-García, Galea e Leiva, 2003). Também, alguns autores investigaram a análise de influência local em modelos de análise de sobrevivência: por exemplo, Pettit e Bin Daud (1989) investigaram a influência local em modelos de Cox com riscos proporcionais; Escobar e Meeker (1992) adaptaram o método de influência local para modelos de regressão com censura; Ortega, Bolfarine e Paula (2003) consideraram o problema da análise de influência local em modelos de regressão loggama generalizado com observações censuradas e mais recentemente, Galea, Leiva e Paula (2004) derivaram as curvaturas da influência local sob vários esquemas de perturbação em modelos de regressão linear log-BS com dados não censurados. Além de considerar a função de verossimilhança para o cálculo das curvaturas, outras sugestões têm sido propostas para lidar com situações não-padrão e para vários modelos, veja por exemplo, Fung e Kwan (1997), Kwan e Fung (1998) e Tanaka, Zhang e Mori (2003), entre outros.

A abordagem original da influência local baseia-se na análise do afastamento pela verossimilhança $L D(\boldsymbol{\omega})=2\left\{l(\widehat{\boldsymbol{\theta}})-l\left(\widehat{\boldsymbol{\theta}}_{\omega}\right)\right\}$, em que $\widehat{\boldsymbol{\theta}}_{\omega}$ denota o estimador de máxima verossimilhança sob o modelo perturbado e $\boldsymbol{\omega}=\left(\omega_{1}, \ldots, \omega_{n}\right)^{\top}$ é o vetor de perturbações aplicadas ao modelo e/ou aos dados. A proposta de Cook (1986) é estudar o comportamento da função $L D(\omega)$, numa vizinhança do vetor de não-perturbação $\omega_{0}$. O procedimento consiste em selecionar uma direção unitária $\ell$, tal que $\|\ell\|=1$, e então, considerar o gráfico de $L D\left(\omega_{0}+a \ell\right)$ contra $a$, com $a \in \mathbb{R}$. Esse gráfico é chamado de linha projetada. Cada linha projetada pode ser caracterizada pela curvatura normal $C_{\ell}(\widehat{\theta})$ em torno de $a=0$ (para mais detalhes ver Souza, 1999).

A sugestão de Cook (1986) é considerar a direção $\ell_{\max }$ correspondente à maior curvatura $C_{\ell_{\max }}$. O gráfico de $\ell_{\max }$ contra o índice das observações pode mostrar os elementos que, sob pequenas perturbações, exercem notável influência sobre $L D(\boldsymbol{\omega})$. Cook (1986) mostra que a 
curvatura normal pode ser expressa na forma geral como $C_{\ell}(\theta)=2\left|\ell^{\top} \Delta^{\top} \ddot{L}_{\theta \theta}^{-1} \Delta \ell\right|$, em que $\Delta$ é uma matriz $(p+q) \times n$, que depende do esquema de perturbação usado, cujos elementos são $\triangle_{i j}=\partial^{2} L(\theta \mid \omega) / \partial \theta_{j} \partial \omega_{i}, i=1, \ldots, p+q$ e $j=1, \ldots, n$, com todas as quantidades sendo avaliadas em $\omega=\omega_{0}$ e $\theta=\widehat{\theta}$. Então, $C_{\ell_{\max }}$ é o maior autovalor da matriz

$$
B=-\Delta^{\top} \ddot{L}_{\theta \theta}^{-1} \Delta,
$$

e $\ell_{\max }$ é o autovetor correspondente (ver, por exemplo, Galea, Leiva e Paula, 2004). Neste contexto, se o interesse é avaliar a influência parcial em um subconjunto de $\boldsymbol{\theta}=\left(\boldsymbol{\theta}_{1}^{\top}, \boldsymbol{\theta}_{2}^{\top}\right)^{\top}$, $\theta_{1}$ por exemplo, temos que a curvatura normal na direção do vetor $\ell$ é dada por

$$
C_{\ell}\left(\theta_{1}\right)=2\left|\ell^{\top} \Delta^{\top}\left(\ddot{L}_{\theta \theta}^{-1}-B_{1}\right) \Delta \ell\right|,
$$

com

$$
B_{1}=\left[\begin{array}{cc}
0 & 0 \\
0 & \ddot{L}_{22}^{-1}
\end{array}\right]
$$

e $\ddot{\boldsymbol{L}}_{22}=\left.\left\{\partial^{2} l(\boldsymbol{\theta} \mid \boldsymbol{\omega}) / \partial \boldsymbol{\theta}_{2}^{\top} \partial \boldsymbol{\theta}_{2}\right\}\right|_{\boldsymbol{\theta}=\hat{\boldsymbol{\theta}}}$.

O gráfico do autovetor associado ao maior autovalor da matriz $-\boldsymbol{\Delta}^{\top}\left(\boldsymbol{L}_{\theta \theta}^{-1}-\boldsymbol{B}_{1}\right) \Delta$ contra o índice das observações pode revelar quais observações estão infuenciando $\widehat{\theta}_{1}$. Analogamente, se o interesse está em $\boldsymbol{\theta}_{2}$, então a curvatura normal na direção do vetor $\boldsymbol{\ell}$ é dada por

$$
C_{\ell}\left(\theta_{2}\right)=2\left|\ell^{\top} \Delta^{\top}\left(\ddot{L}_{\theta \theta}^{-1}-B_{2}\right) \Delta \ell\right|
$$

com

$$
\boldsymbol{B}_{2}=\left[\begin{array}{cc}
\ddot{\boldsymbol{L}}_{11}^{-1} & 0 \\
0 & 0
\end{array}\right]
$$

e $\ddot{\boldsymbol{L}}_{11}=\left.\left\{\partial^{2} l(\boldsymbol{\theta} \mid \boldsymbol{\omega}) / \partial \boldsymbol{\theta}_{1}^{\top} \partial \boldsymbol{\theta}_{1}\right\}\right|_{\boldsymbol{\theta}=\hat{\boldsymbol{\theta}}}$.

A influência local das observações em $\widehat{\theta}_{2}$ pode ser avaliada considerando o gráfico $\ell_{\max }$ para a matriz $-\Delta^{\top}\left(\ddot{L}_{\theta \theta}^{-1}-B_{2}\right) \Delta$ contra o índice das observações. Lesaffre e Verbeke (1998) sugerem considerar também a curvatura na direção da $i$-ésima observação, ou seja, calcular a curvatura na direção de $\ell_{i}$, em que $\ell_{i}$ é um vetor $n \times 1$ de zeros com um na $i$-ésima posição. Logo, a curvatura na direção de $\ell_{i}$ assume a forma $C_{i}=2\left|\Delta_{i}^{\top} \ddot{L}_{\theta \theta}^{-1} \Delta_{i}\right|$, em que $\Delta_{i}^{\top}$ denota a $i$-ésima linha de $\Delta$. Verbeke e Molenberghs (2000, Seção 11.3) propõem considerar como influentes os casos em que

$$
C_{i} \geq 2 \bar{C}
$$

sendó $\bar{C}=\frac{1}{n} \sum_{i=1}^{n} C_{i}$. Para o modelo de regressão linear log-BS os elementos de $\ddot{L}_{\widehat{\theta} \widehat{\theta}}$ são $\ddot{L}_{\widehat{\beta} \widehat{\beta}}=X^{\top} \widehat{V} \boldsymbol{X}, \ddot{\boldsymbol{L}}_{\widehat{\beta} \widehat{\alpha}}=X^{\top} \widehat{\boldsymbol{K}}$ e $\ddot{\boldsymbol{L}}_{\widehat{\alpha} \widehat{\alpha}}=\operatorname{tr}(\widehat{\boldsymbol{G}})$, dados em (2.5). 
Derivamos as curvaturas normais para o modelo de regressão linear log-BS com observações censuradas sob três esquemas de perturbação. São eles: ponderação de casos, perturbação na variável resposta e perturbação na variável explicativa. Na próxima seção, apresentaremos a matriz $\Delta$ para cada esquema de perturbação considerado.

\subsubsection{Cálculo das curvaturas}

Nesta seção obteremos, para o esquema de perturbação indicado, a matriz

$$
\Delta=\left(\Delta_{i j}\right)_{(p+1) \times n}=\left(\frac{\partial^{2} l(\boldsymbol{\theta} \mid \boldsymbol{\omega})}{\partial \theta_{i} \partial \omega_{j}}\right), i=1, \ldots, p+1 \text { e } j=1, \ldots, n,
$$

considerando o modelo definido em (2.1) e o logaritmo da função de verossimilhança dado em (2.2).

\section{Ponderação de casos}

Consideremos o vetor de pesos $\omega=\left(\omega_{1}, \ldots, \omega_{n}\right)^{\top}$ e o logaritmo da função de verossimilhança perturbada

$$
l(\boldsymbol{\theta} \mid \omega) \propto \sum_{i \in D} \omega_{i}\left\{\log \left(\xi_{i 1}\right)-\frac{1}{2}\left[\log (8 \pi)+\xi_{i 2}^{2}\right]\right\}+\sum_{i \in C} \omega_{i} \log \left[1-\Phi\left(\xi_{i 2}\right)\right],
$$

em que $0 \leq \omega_{i} \leq 1$ e $\omega_{0}=(1, \ldots, 1)^{\top}$. A matriz $\Delta$ é dada por

$$
\Delta=\left(\begin{array}{c}
\Delta_{\beta} \\
\Delta_{\alpha}
\end{array}\right)
$$

em que $\Delta_{\beta}$ é uma matriz $p \times n$ expressa como $\Delta_{\beta}=X^{\top} \operatorname{diag}\left\{\widehat{b}_{1}, \ldots, \widehat{b}_{n}\right\}$ com

$$
\widehat{b}_{i}= \begin{cases}\frac{1}{2}\left(\widehat{\xi}_{i 1} \widehat{\xi}_{i 2}-\widehat{\xi}_{i 2} / \widehat{\xi}_{i 1}\right), & \text { se } i \in D \\ \frac{\widehat{\xi}_{i 1}}{2} h\left(\widehat{\xi}_{i 2}\right), & \text { se } i \in C\end{cases}
$$

e $\Delta_{\alpha}=\left(\widehat{a}_{1}, \ldots, \widehat{a}_{n}\right), \mathrm{com}$

$$
\widehat{a}_{i}= \begin{cases}-\frac{1}{\widehat{\alpha}}+\frac{\left(\widehat{\xi}_{i 2}\right)^{2}}{\widehat{\alpha}}, & \text { se } i \in D \\ \widehat{\xi}_{i 2} h\left(\widehat{\xi}_{i 2}\right), & \text { se } i \in C\end{cases}
$$

em que $h(\cdot)$ é dada em (2.4) e $\widehat{\xi}_{i 1}, \widehat{\xi}_{i 2}$ são as estimativas de máxima verossimilhança de $\xi_{i 1}$ e $\xi_{i 2}$ dadas em (2.2). 


\section{Perturbação na variável resposta}

Consideremos aqui que cada $y_{i}$ é perturbado da seguinte forma: $y_{i \omega}=y_{i}+\omega_{i} S_{y}$, em que $S_{y}$ é um fator de escala que pode ser estimado pelo desvio padrão de $Y$ e $\omega_{i} \in \mathbb{R}$. O logaritmo da função de verossimilhança perturbada assume a forma

$$
l(\boldsymbol{\theta} \mid \boldsymbol{\omega}) \propto \sum_{i \in D}\left\{\log \left(\xi_{i 1 \omega_{1}}\right)-\frac{1}{2}\left[\log (8 \pi)+\xi_{i 2 \omega_{1}}^{2}\right]\right\}+\sum_{i \in C} \log \left[1-\Phi\left(\xi_{i 2 \omega_{1}}\right)\right],
$$

em que $\xi_{i 1 \omega_{1}}=\frac{2}{\alpha} \cosh \left(\frac{y_{i \omega}-\mu_{i}}{2}\right), \xi_{i 2 \omega_{1}}=\frac{2}{\alpha} \operatorname{senh}\left(\frac{y_{i \omega}-\mu_{i}}{2}\right)$ e $\omega_{0}=(0, \ldots, 0)^{\top}$. A matriz $\Delta$ assume a mesma forma dada em (2.12), em que

$$
\Delta_{\beta}=\boldsymbol{X}^{\top} \operatorname{diag}\left\{\widehat{d}_{1}, \ldots, \widehat{d}_{n}\right\}
$$

com

$$
\widehat{d}_{i}= \begin{cases}S_{y}\left[\frac{1}{\widehat{\alpha}^{2}} \cosh \left(y_{i}-\widehat{\mu}_{i}\right)-\frac{1}{4} \operatorname{sech}^{2}\left(\frac{y_{i}-\widehat{\mu}_{i}}{2}\right)\right], & \text { se } i \in D \\ \frac{S_{y}}{4}\left[\widehat{\xi}_{i 2} h\left(\widehat{\xi}_{i 2}\right)+\left(\widehat{\xi}_{i 1}\right)^{2} h^{\prime}\left(\widehat{\xi}_{i 2}\right)\right], & \text { se } i \in C\end{cases}
$$

e $\Delta_{\alpha}=\left(\widehat{c}_{1}, \ldots, \widehat{c}_{n}\right) \mathrm{com}$

$$
\widehat{c}_{i}= \begin{cases}\frac{S_{y}}{\widehat{\alpha}} \widehat{\xi}_{i 1} \widehat{\xi}_{i 2}, & \text { se } i \in D ; \\ \frac{S_{y}}{2 \widehat{\alpha}}\left[\widehat{\xi}_{i 1} h\left(\widehat{\xi}_{i 2}\right)+\widehat{\xi}_{i 1} \widehat{\xi}_{i 2} h^{\prime}\left(\widehat{\xi}_{i 2}\right)\right], & \text { se } i \in C ;\end{cases}
$$

em que $h(\cdot)$ e $h^{\prime}(\cdot)$ são dados em (2.4), $\widehat{\xi}_{i 1}$ e $\widehat{\xi}_{i 2}$ são $\xi_{i 1}$ e $\xi_{i 2}$ dados em (2.15), avaliados $\widehat{\boldsymbol{\theta}}=\left(\widehat{\alpha}, \widehat{\boldsymbol{\beta}}^{\top}\right)^{\top}$ e $\boldsymbol{\omega}_{0}$.

\section{Perturbação em uma variável explicativa}

Consideremos agora uma perturbação aditiva de uma variável explicativa particular, digamos $\boldsymbol{x}_{t}$, fazendo $x_{i t \omega}=x_{i t}+\omega_{i} S_{x}$, em que $S_{x}$ é um fator de escala que pode ser estimado pelo desvio padrão de $x_{t}$ e $\omega_{i} \in \mathbb{R}, t \in\{1, \ldots, p\}$. O logaritmo da função de verossimilhança perturbada toma a forma

$$
l(\boldsymbol{\theta} \mid \boldsymbol{\omega}) \propto \sum_{i \in D}\left\{\log \left(\xi_{i 1 \omega_{2}}\right)-\frac{1}{2}\left[\log (8 \pi)+\xi_{i 2 \omega_{2}}^{2}\right]\right\}+\sum_{i \in C} \log \left[1-\Phi\left(\xi_{i 2 \omega_{2}}\right)\right],
$$

em que $\xi_{i 1 \omega_{2}}=\frac{2}{\alpha} \cosh \left(\frac{y_{i}-\mu_{i}-\beta_{t} \omega_{i} S_{x}}{2}\right), \xi_{i 2 \omega_{2}}=\frac{2}{\alpha} \operatorname{senh}\left(\frac{y_{i}-\mu_{i}-\beta_{t} \omega_{i} S_{x}}{2}\right)$ e $\omega_{0}=(0, \ldots, 0)^{\top}$. A matriz $\Delta$ assume a forma dada em (2.12), em que $\Delta_{\beta}$ é uma matriz $p \times n$ cujos elementos $\Delta_{\beta_{i j}}$ quando $j \neq t$ são dados por

$$
\Delta_{\beta_{i j}}= \begin{cases}S_{x} \widehat{\beta}_{t} x_{i j}\left[\frac{1}{4} \operatorname{sech}^{2}\left(\frac{y_{i}-\widehat{\mu}_{i}}{2}\right)-\frac{1}{\widehat{\alpha}^{2}} \cosh \left(y_{i}-\widehat{\mu}_{i}\right)\right], & \text { se } i \in D ; \\ -\frac{S_{x} \widehat{\beta}_{t} x_{i j}}{4}\left[\widehat{\xi}_{i 2} h\left(\widehat{\xi}_{i 2}\right)+\left(\widehat{\xi}_{i 1}\right)^{2} h^{\prime}\left(\widehat{\xi}_{i 2}\right)\right], & \text { se } i \in C ;\end{cases}
$$


e quando $j=t$

$$
\Delta_{\beta_{i t}}= \begin{cases}S_{x} \widehat{\beta}_{t} x_{i t}\left[\frac{1}{4} \operatorname{sech}^{2}\left(\frac{y_{i}-\widehat{\mu}_{i}}{2}\right)-\frac{1}{\widehat{\alpha}^{2}} \cosh \left(y_{i}-\widehat{\mu}_{i}\right)\right] & \\ +S_{x}\left[\frac{1}{\widehat{\alpha}^{2}} \operatorname{senh}\left(y_{i}-\widehat{\mu}_{i}\right)-\frac{1}{2} \tanh \left(\frac{y_{i}-\widehat{\mu}_{i}}{2}\right)\right], & \text { se } i \in D \\ -\frac{S_{x} \widehat{\beta}_{t} x_{i t}}{4}\left[\widehat{\xi}_{i 2} h\left(\widehat{\xi}_{i 2}\right)+\left(\widehat{\xi}_{i 1}\right)^{2} h^{\prime}\left(\widehat{\xi}_{i 2}\right)\right]+\frac{S_{x}}{2} \widehat{\xi}_{i 1} h\left(\widehat{\xi}_{i 2}\right), & \text { se } i \in C\end{cases}
$$

e $\Delta_{\alpha}=\left(\widehat{\varphi}_{1}, \ldots, \widehat{\varphi}_{n}\right)$, com

$$
\widehat{\varphi}_{i}= \begin{cases}-\frac{2}{\widehat{\alpha}^{3}} S_{x} \widehat{\beta}_{t} \operatorname{senh}\left(y_{i}-\widehat{\mu}_{i}\right), & \text { se } i \in D \\ -\frac{\widehat{\beta}_{t} S_{x}}{2 \widehat{\alpha}}\left[\widehat{\xi}_{i 1} h\left(\widehat{\xi}_{i 2}\right)+\frac{2}{\widehat{\alpha}^{2}} \operatorname{senh}\left(y_{i}-\widehat{\mu}_{i}\right) h^{\prime}\left(\widehat{\xi}_{i 2}\right)\right], & \text { se } i \in C\end{cases}
$$

em que $h(\cdot)$ e $h^{\prime}(\cdot)$ são dados em (2.4), $\widehat{\xi}_{i 1}$ e $\widehat{\xi}_{i 2}$ são $\xi_{i 1}$ e $\xi_{i 2}$ dados em (2.16), avaliados $\widehat{\boldsymbol{\theta}}=\left(\widehat{\alpha}, \widehat{\boldsymbol{\beta}}^{\top}\right)^{\top}$ e $\boldsymbol{\omega}_{0}$.

\section{Ponderação de casos não censurados}

Suponhamos que apenas os casos não censurados sejam perturbados, isto significa assumir $\omega_{i}=1$ para $i \in C$ na expressão (2.11). Então, as quantidades $\widehat{b}_{i}$ e $\widehat{a}_{i}$ dadas em (2.13) e (2.14) se reduzem, respectivamente, a

$$
\widehat{b}_{i}= \begin{cases}\frac{1}{2}\left(\widehat{\xi}_{i 1} \widehat{\xi}_{i 2}-\widehat{\xi}_{i 2} / \widehat{\xi}_{i 1}\right), & \text { se } i \in D \\ 0, & \text { se } i \in C\end{cases}
$$

e

$$
\widehat{a}_{i}= \begin{cases}-\frac{1}{\widehat{\alpha}}+\frac{\left(\widehat{\xi}_{i 2}\right)^{2}}{\widehat{\alpha}}, & \text { se } i \in D \\ 0, & \text { se } i \in C\end{cases}
$$

em que $\widehat{\xi}_{i 1}$ e $\widehat{\xi}_{i 2}$ são as estimativas de máxima verossimilhança de $\xi_{i 1}$ e $\xi_{i 2}$ dadas em (2.2). Note que as expressões acima para $i \in D$ são as mesmas obtidas por Galea, Leiva e Paula (2004).

\section{Ponderação de casos censurados}

Suponhamos agora que apenas os casos censurados são perturbados, isto é, $\omega_{i}=1$ para $i \in D$ na expressão (2.11). Neste caso, as quantidades $\widehat{b}_{i}$ e $\widehat{a}_{i}$ dadas em (2.13) e (2.14) se reduzem, respectivamente, a

$$
\widehat{b}_{i}= \begin{cases}0, & \text { se } i \in D \\ \frac{\widehat{\xi}_{i 1}}{2} h\left(\widehat{\xi}_{i 2}\right), & \text { se } i \in C\end{cases}
$$


e

$$
\widehat{a}_{i}= \begin{cases}0, & \text { se } i \in D \\ \frac{\widehat{\xi}_{i 2}}{\widehat{\alpha}} h\left(\widehat{\xi}_{i 2}\right), & \text { se } i \in C ;\end{cases}
$$

em que $h(\cdot)$ é dada em $(2.4)$ e $\widehat{\xi}_{i 1}, \widehat{\xi}_{i 2}$ são as estimativas de máxima verossimilhança de $\xi_{i 1}$ e $\xi_{i 2}$ dadas em (2.2).

\subsection{Análise de resíduos}

A análise de resíduos é uma ferramenta importante para a validação do modelo. Através da análise de resíduos podemos verificar se existem afastamentos sérios das suposições feitas para o modelo, bem como a existência de observações extremas. Nesta seção, consideraremos dois tipos de resíduos: resíduo componente do desvio (ver, por exemplo, McCullagh e Nelder, 1989) e resíduo tipo martingale (ver, por exemplo, Barlow e Prentice, 1988 e Therneau, Grambsch e Fleming, 1990). Mais detalhes podem ser encontrados em Ortega, Bolfarine e Paula (2003).

\section{Resíduo componente do desvio}

O resíduo componente do desvio é definido por

$$
r_{D C_{i}}=\operatorname{sinal}\left(y_{i}-\hat{\mu}_{i}\right) \sqrt{2}\left[l_{i}(\widetilde{\theta})-l_{i}(\widehat{\theta})\right]^{\frac{1}{2}},
$$

em que $\widetilde{\theta}$ é o estimador de máxima verossimilhança de $\boldsymbol{\theta}$ sob o modelo saturado (com $n$ parâmetros), $\widehat{\theta}$ é o estimador de máxima verossimilhança de $\theta$ sob o modelo de interesse (com $p$ parâmetros) e sinal $\left(y_{i}-\hat{\mu}_{i}\right)$ significa o sinal de $\left(y_{i}-\hat{\mu}_{i}\right)$. Davison e Gigli (1989) definem o resíduo componente do desvio para dados censurados como

$$
r_{D C_{i}}=\operatorname{sinal}\left(y_{i}-\hat{\mu}_{i}\right)\left\{-2 \log \left[S_{Y}\left(y_{i}, \widehat{\theta}\right)\right]\right\}^{\frac{1}{2}}
$$

em que $S_{Y}\left(y_{i}, \widehat{\boldsymbol{\theta}}\right)$ é o estimador de máxima verossimilhança da função de sobrevivência (ver, por exemplo, Ortega, 2001). Portanto, considerando $\alpha$ fixo ou conhecido e $\alpha<2$, temos que o resíduo componente do desvio para o modelo de regressão linear log-Birnbaum-Saunders fica dado por

$$
r_{D C_{i}}= \begin{cases}\operatorname{sinal}\left(y_{i}-\hat{\mu}_{i}\right) \sqrt{2}\left[-\log \left(\cosh \left[\frac{y_{i}-\hat{\mu}_{i}}{2}\right]\right)+\frac{2}{\hat{\alpha}^{2}} \operatorname{senh}^{2}\left(\frac{y_{i}-\hat{\mu}_{i}}{2}\right)\right]^{\frac{1}{2}}, & \text { se } i \in D ; \\ \operatorname{sinal}\left(y_{i}-\hat{\mu}_{i}\right)\left[-2 \log \left(1-\Phi\left[\frac{2}{\hat{\alpha}} \operatorname{senh}\left(\frac{y_{i}-\hat{\mu}_{i}}{2}\right)\right]\right)\right]^{\frac{1}{2}}, & \text { se } i \in C,\end{cases}
$$

$i=1, \ldots, n$. 


\section{Resíduo tipo martingale}

Therneau, Grambsch e Fleming (1990) propuseram o resíduo componente do desvio em processos de contagem usando basicamente resíduos martingales. Os resíduos martingales são assimétricos, assumem valor máximo em +1 e valor mínimo em $-\infty$. Na área paramétrica, os resíduos martingales podem ser expressos como $r_{M_{i}}=\delta_{i}+\log \left[S_{Y}\left(y_{i}, \widehat{\theta}\right)\right]$, com $\delta_{i}=0$ indicando se a observação é censurada e $\delta_{i}=1$ indicando se a observação é não censurada (ver, por exemplo, Klein e Moeschberger, 1997, e Ortega, Bolfarine e Paula, 2003). Então, de acordo com (1.9), o resíduo martingale para os modelos de regressão linear log-BS assume a forma

$$
r_{M_{i}}=\delta_{i}+\log \left\{1-\Phi\left[\frac{2}{\widehat{\alpha}} \operatorname{senh}\left(\frac{y_{i}-\widehat{\mu}_{i}}{2}\right)\right]\right\} .
$$

O resíduo componente do desvio proposto por Therneau, Grambsch e Fleming (1990) é uma transformação do resíduo martingale com a finalidade de atenuar a assimetria. Essa transformação foi motivada pelos resíduos componente do desvio encontrados em modelos lineares generalizados. Em particular, o resíduo componente do desvio para o modelo de Cox com variáveis explicativas não dependendo do tempo é expresso como

$$
r_{M D_{i}}=\operatorname{sinal}\left(r_{M_{i}}\right)\left\{-2\left[r_{M_{i}}+\delta_{i} \log \left(\delta_{i}-r_{M_{i}}\right)\right]\right\}^{\frac{1}{2}},
$$

em que $r_{M_{i}}$ é o resíduo martingale, $i=1, \ldots, n$.

Usaremos os resíduos $r_{M D_{i}}$ como uma transformação dos resíduos martingales com o fim de obtermos resíduos distribuídos simetricamente em torno do zero. Chamaremos este resíduo de resíduo tipo martingale.

\section{Resíduos padronizados}

Ortega (2001) sugere padronizar o resíduo componente do desvio $r_{D C_{i}}$ e o resíduo tipo martingale $r_{M D_{i}}$ para dados censurados como

$$
r_{D C_{i}}^{*}=\frac{r_{D C_{i}}}{\sqrt{1-G L_{i i}}}
$$

e

$$
r_{M D_{i}}^{*}=\frac{r_{M D_{i}}}{\sqrt{1-G L_{i i}}}
$$

sendo $G L_{i i}$ o $i$-ésimo elemento da diagonal principal da matriz de pontos de alavanca generalizada (ver Wei, Hu e Fung, 1998) definida por

$$
G L(\theta)=D_{\theta}\left(-\ddot{L}_{\theta \theta}\right)^{-1} \ddot{\boldsymbol{L}}_{\theta y}
$$


em que $\boldsymbol{D}_{\theta}=\partial \boldsymbol{\mu} / \partial \boldsymbol{\theta}^{\top}=(\boldsymbol{X}, \mathbf{0}), \ddot{\boldsymbol{L}}_{\theta \theta}$ é dada em (2.5) e

$$
\ddot{\boldsymbol{L}}_{\theta y}=\left(\begin{array}{c}
\ddot{\boldsymbol{L}}_{\beta y} \\
\ddot{\boldsymbol{L}}_{\alpha y}
\end{array}\right),
$$

em que $\ddot{\boldsymbol{L}}_{\beta y}=-\boldsymbol{X}^{T} \boldsymbol{V}, \operatorname{com} \boldsymbol{V}=\operatorname{diag}\left\{v_{1}(\boldsymbol{\theta}), \ldots, v_{n}(\boldsymbol{\theta})\right\}, v_{i}(\boldsymbol{\theta})$ é dado em (A.12) e

$$
\ddot{\boldsymbol{L}}_{\alpha y_{i}}= \begin{cases}\frac{1}{\alpha} \xi_{i 1} \xi_{i 2}, & \text { se } i \in D ; \\ \frac{1}{2 \alpha} \xi_{i 1} h\left(\xi_{i 2}\right)+\frac{1}{2 \alpha} \xi_{i 1} \xi_{i 2} h^{\prime}\left(\xi_{i 2}\right), & \text { se } i \in C ;\end{cases}
$$

com todas as expressões avaliadas em $\theta=\widehat{\theta}$. O gráfico de $G L_{i i}$ contra o índice das observações pode revelar observações com alta influência no seu próprio valor predito.

\section{Simulações}

Com o objetivo de investigar o comportamento da distribuição empírica dos resíduos $r_{D C_{i}}^{*}$ e $r_{M D_{i}}^{*} \mathrm{p}$ a os valores de $n=30,50,100, \alpha=0,5 ; 1,0 ; 1,5$ e proporção de censura $p c=0,10 ; 0,30 ; 0,50$, realizamos um pequeno estudo de simulação descrito como segue. Consideramos uma única variável explicativa $X$ a qual segue uma distribuição uniforme $U(0,1)$. As observações Birnbaum-Saunders foram geradas através de observações normais usando a expressão

$$
T_{i}=\eta_{i}\left[1+\frac{\alpha^{2} Z_{i}^{2}}{2}+\alpha Z_{i}\left(1+\frac{\alpha^{2} Z_{i}^{2}}{4}\right)^{\frac{1}{2}}\right],
$$

em que $Z_{i} \sim N(0,1)$ e $\eta_{i}=\exp \left(\beta_{0}+\beta_{1} x_{i}\right)$, com $\beta_{0}=4$ e $\beta_{1}=-2$, para $i=1, \ldots, n$. Seguindo o procedimento apresentado por Wang, Desmond e Lu (2006) geramos as variáveis aleatórias censuradas $C_{i}$ da distribuição $\log$-normal com fda $\Phi\left(\frac{\log u-\mu}{\sigma}\right)$, em que $\Phi(\cdot)$ é a fda da distribuição normal padrão. Fixamos $\sigma$ igual a 1 e variamos $\mu$ até obter as diferentes proporções de censura. Assim, os tempos observados foram $\min \left(T_{i}, C_{i}\right)$ e o indicador de censura é $\delta_{i}=1_{\left(T_{i} \leq C_{i}\right)}$, sendo $1_{(\cdot)}$ a função indicadora. Para combinações de $n, \alpha$ e $p c$ foram geradas 1000 amostras aleatórias. Para cada amostra, o modelo de regressão linear log-BS dado em (2.1), em que $Y_{i}=\log \left\{\min \left(T_{i}, C_{i}\right)\right\}, \operatorname{com} i=1, \ldots, n$, foi ajustado. Então, obtemos os

resíduos $r_{D C_{i}}^{*}$ e $r_{M D_{i}}^{*}$ para cada amostra gerada. Ordenamos os resíduos e construímos gráficos normais de probabilidade entre os percentis médios dos resíduos e os percentis esperados da distribuição normal padrão. As Figuras 2.1-2.3 mostram os gráficos normais de probabilidade para os resíduos componente do desvio padronizado. Os gráficos para os resíduos tipo martingale padronizados foram omitidos, pois apresentam comportamentos similares. A principal conclusão obtida a partir dos gráficos gerados é a de que as distribuições empíricas dos dois resíduos apresentam uma boa concordância com a distribuição normal padrão para cada $n, \alpha$ 
e proporção de censura escolhida. Em particular, quando a proporção de censura decresce, as distribuições empíricas dos resíduos se aproximam mais rapidamente da distribuição normal padrão. O mesmo ocorre quando o parâmetro $\alpha$ decresce. Porém, a extensão desses resultados para situações mais gerais não é direta. O uso dos gráficos normais de probabilidades para $r_{D C_{i}}^{*}$ e $r_{M D_{i}}^{*}$ com envelopes seria o recomendado (ver Atkinson, 1981 e Williams, 1987). Os envelopes são bandas de confiança obtidas por métodos de Monte Carlo a partir do modelo ajustado para avaliar afastamentos sérios da distribuição postulada para os resíduos e de forma indireta da distribuição postulada para os erros. Gráficos dos resíduos contra os valores ajustados e os valores das variáveis explicativas podem ser úteis para detectar pontos extremos e alguma tendência sistemática, embora a interpretação não seja necessariamente a mesma da usada em modelos de regressão normal linear. Os resultados apresentados neste capítulo estão também descritos em Leiva et al. (2006).

\subsubsection{Aplicações}

\section{Estudo dos tempos de sobrevivência de pacientes com mieloma múltiplo}

Analisamos inicialmente o conjunto de dados considerado em Krall, Uthoff e Harley. (1975) e apresentado em Lawless (1982, p. 332-333), sobre um estudo que trata dos tempos de sobrevivência, em meses, de 65 pacientes com mieloma múltiplo. Esse conjunto de dados foi analisado recentemente por Jin et al. (2003) e Ghosh e Ghosal (2006) que usaram um modelo semiparamétrico de tempo de falha acelerado. O principal objetivo deste estudo é relatar o tempo de sobrevivência $(t)$ ao mieloma múltiplo com um número de variáveis de prognóstico com dados censurados. Os dados apresentam os tempos de sobrevivência, em meses, para 65 pacientes que foram tratados com agentes alkylating dos quais 48 morreram durante o estudo e apenas 17 sobreviveram e também apresentam as seguintes variáveis de prognóstico: logaritmo da medida de uréia nitrogenada no sangue no diagnóstico $\left(x_{1}\right)$; medida de hemoglobina no diagnóstico $\left(x_{2}\right)$; idade no diagnóstico $\left(x_{3}\right)$; sexo $\left(x_{4}, 0\right.$ : masculino e 1: feminino) e medida de cálcio sérico no diagnóstico $\left(x_{5}\right)$.

Em muitos problemas médicos (por exemplo, doenças cardíacas ou diferentes tipos de câncer), um tipo de dano acumulado provocado por vários fatores de risco é detectado. Então, essa degradação leva a um processo de fadiga, cuja propagação do tempo de sobrevivência pode ser adequadamente modelada pela distribuição Birnbaum-Saunders. Assim, sob este argumento, propomos o modelo log-Birnbaum-Saunders com o objetivo de analisar esse conjunto de dados. 

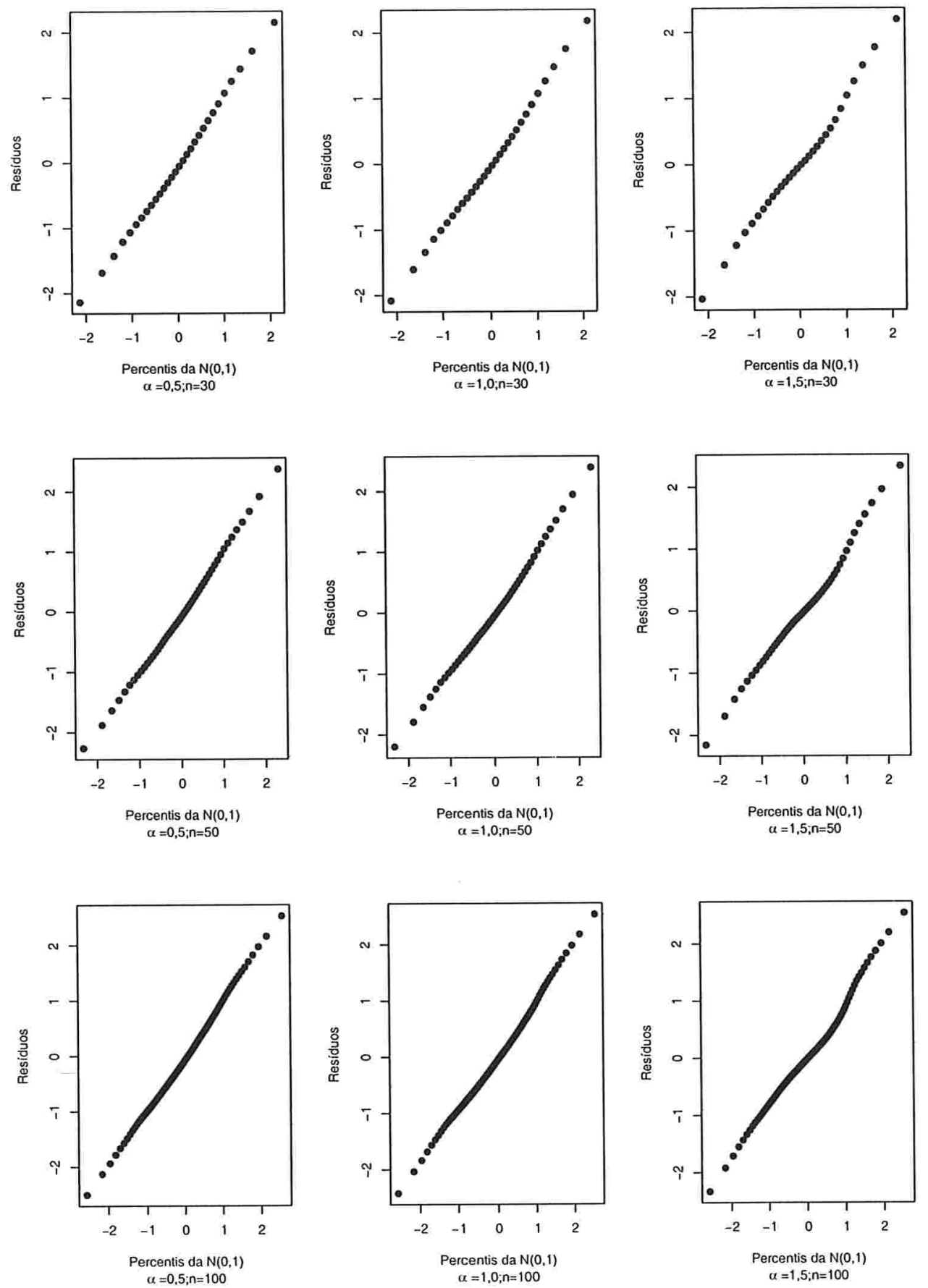

Figura 2.1: gráficos normais de probabilidade para os resíduos componente do desvio padronizado com porcentagem de censura de $10 \%$. 

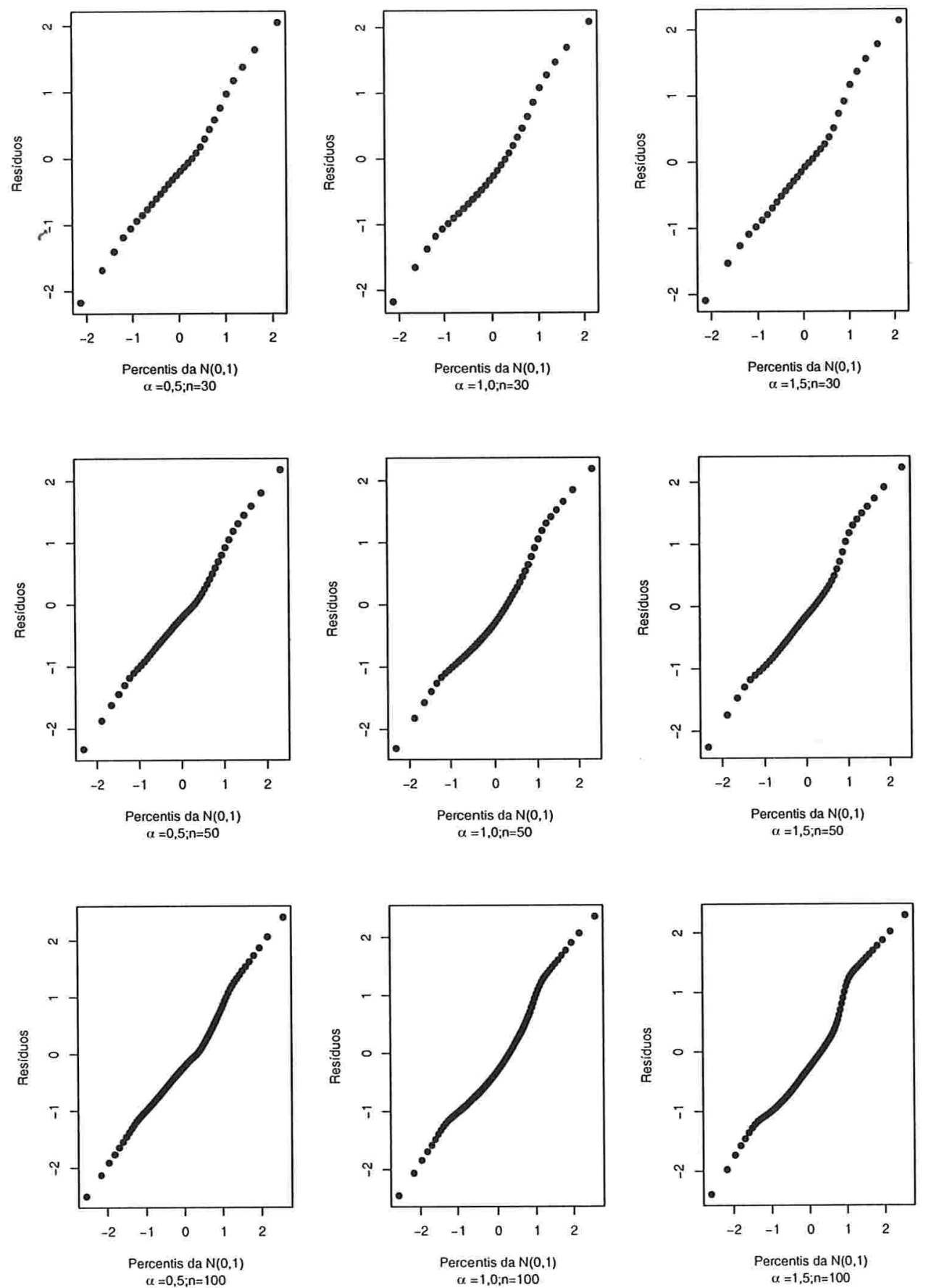

Figura 2.2: gráficos normais de probabilidade para os resíduos componente do desvio padronizado com porcentagem de censura de $30 \%$. 

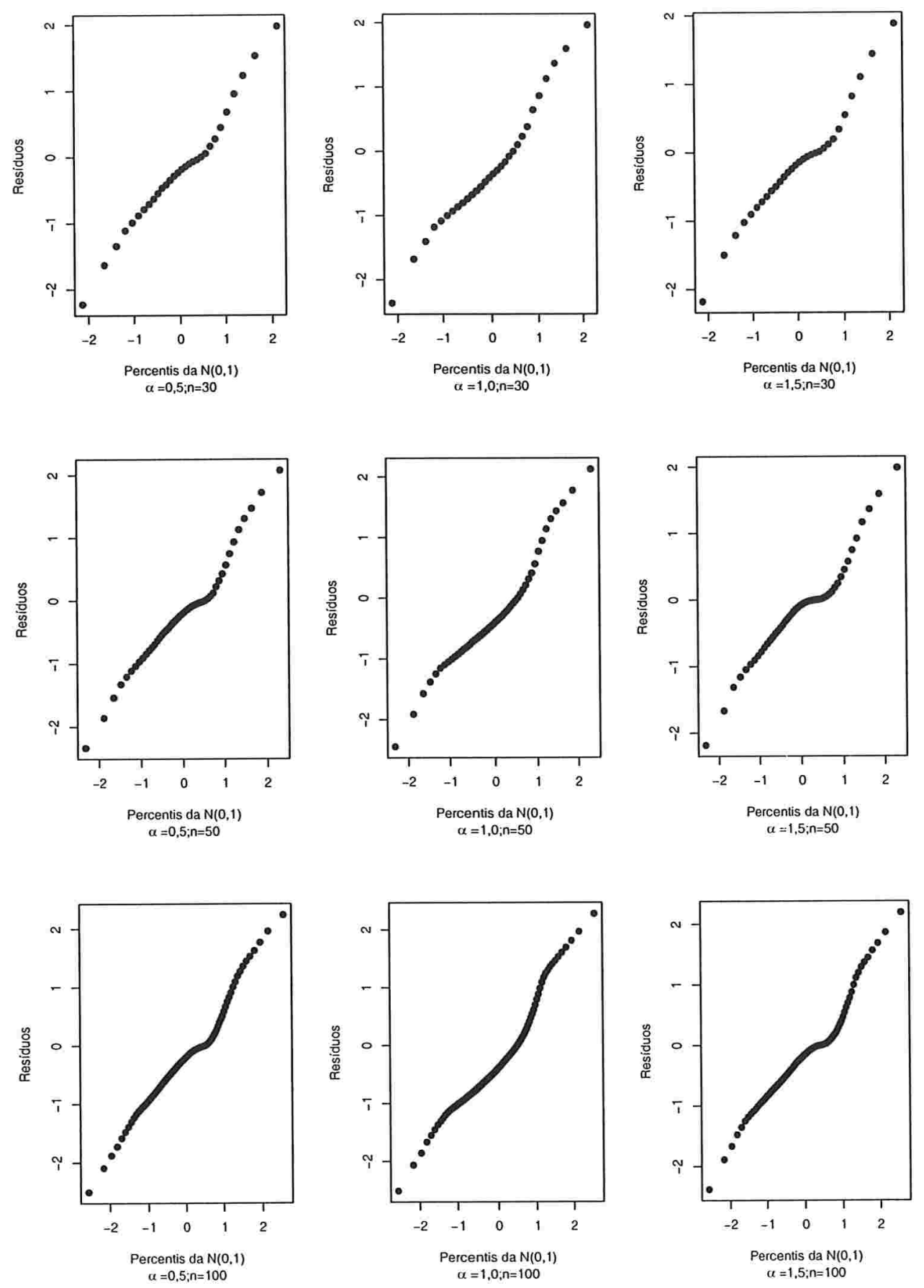

Figura 2.3: gráficos normais de probabilidade para os resíduos componente do desvio padronizado com porcentagem de censura de $50 \%$. 
Consideremos o seguinte modelo de regressão:

$$
y_{i}=\beta_{0}+\beta_{1} x_{i 1}+\beta_{2} x_{i 2}+\beta_{3} x_{i 3}+\beta_{4} x_{i 4}+\beta_{5} x_{i 5}+\varepsilon_{i}, i=1, \ldots, 65
$$

em que $\varepsilon_{i}$ são variáveis aleatórias independentes e identicamente distribuídas tais que $\varepsilon_{i} \sim$ $S N(\alpha, 0,2)$ e a resposta $y_{i}$ denota o logaritmo do tempo de sobrevivência. As estimativas de máxima verossimilhança de $\boldsymbol{\theta}=\left(\boldsymbol{\beta}^{\top}, \alpha\right)^{\top}=\left(\beta_{0}, \beta_{1}, \ldots, \beta_{5}, \alpha\right)^{\top}$ são (erro padrão aproximado entre parênteses):

$$
\begin{gathered}
\widehat{\beta}_{0}=4,500(1,282), \widehat{\beta}_{1}=-1,596(0,435), \widehat{\beta}_{2}=0,142(0,050), \widehat{\beta}_{3}=0,010(0,013), \\
\widehat{\beta}_{4}=0,209(0,284), \widehat{\beta}_{5}=-0,141(0,069) \text { e } \widehat{\alpha}=1,082(0,112) .
\end{gathered}
$$

Notemos que as variáveis explicativas $x_{3}$ e $x_{4}$ são marginalmente não significativas a um nível de significância de $10 \%$.

Com o objetivo de verificar a existência de observações extremas e de alta alavanca bem como detectar possíveis afastamentos das suposições feitas para o modelo, apresentamos na Figura 2.4 gráficos dos resíduos $r_{D C_{i}}^{*}$ e $r_{M D_{i}}^{*}$ contra os valores ajustados, respectivamente, e o gráfico de pontos de alavanca generalizada na Figura 2.5. Como podemos notar, nas Figuras 2.4(a) e 2.4(b) não há presença de observações extremas assim como não há indicação de nenhuma tendência sistemática. Na Figura 2.5 a observação \#2 se destaca como ponto de alta alavanca. Essa observação corresponde ao paciente mais jovem, com 38 anos de idade, do sexo masculino e que sobreviveu apenas um mês ao tratamento. Esse paciente apresenta taxas de uréia nitrogenada do sangue (em logaritmo) e hemoglobina maiores que a média dos 65 pacientes e a taxa de cálcio sérico muito alta, a maior entre os pacientes. Os gráficos normais de probabilidade dos resíduos $r_{D C_{i}}^{*}$ e $r_{M D_{i}}^{*}$ com os envelopes gerados são apresentados na Figura 2.6. Como podemos observar, pelos gráficos, não há índicios de afastamento da suposição de que os erros têm distribuição log-BS.

As Figuras 2.7-2.10 mostram os gráficos de $\left|l_{\max }\right|$ contra o índice das observações para $\boldsymbol{\theta}$, $\boldsymbol{\beta}$ e $\alpha$, em que $\boldsymbol{\theta}=\left(\theta_{1}^{\top}, \theta_{2}\right)^{\top}$, com $\theta_{1}=\boldsymbol{\beta}=\left(\beta_{0}, \ldots, \beta_{5}\right)^{\top}$ e $\theta_{2}=\alpha$, sob cada esquema de perturbação indicado. Como podemos constatar nessas figuras, a observação \#40 se destaca como a mais influente em todos os gráficos. Essa observação se refere a um paciente do sexo masculino, com 74 anos de idade e que sobreviveu 51 meses ao tratamento. As taxas de uréia nitrogenada do sangue e cálcio sérico são maiores que a média dos 65 pacientes e a taxa de hemoglobina é menor que a média dos 65 pacientes. Outras observações se destacaram com alguma influência sobre as estimativas dos parâmetros. Por exemplo, as observações $\# 3$, \#5, \#44 e \#48 se destacam como potencialmente influentes nas estimativas $\widehat{\boldsymbol{\beta}}$ e $\widehat{\alpha}$ sob cada esquema de perturbação usado. Quando perturbamos apenas os casos censurados, detectamos 

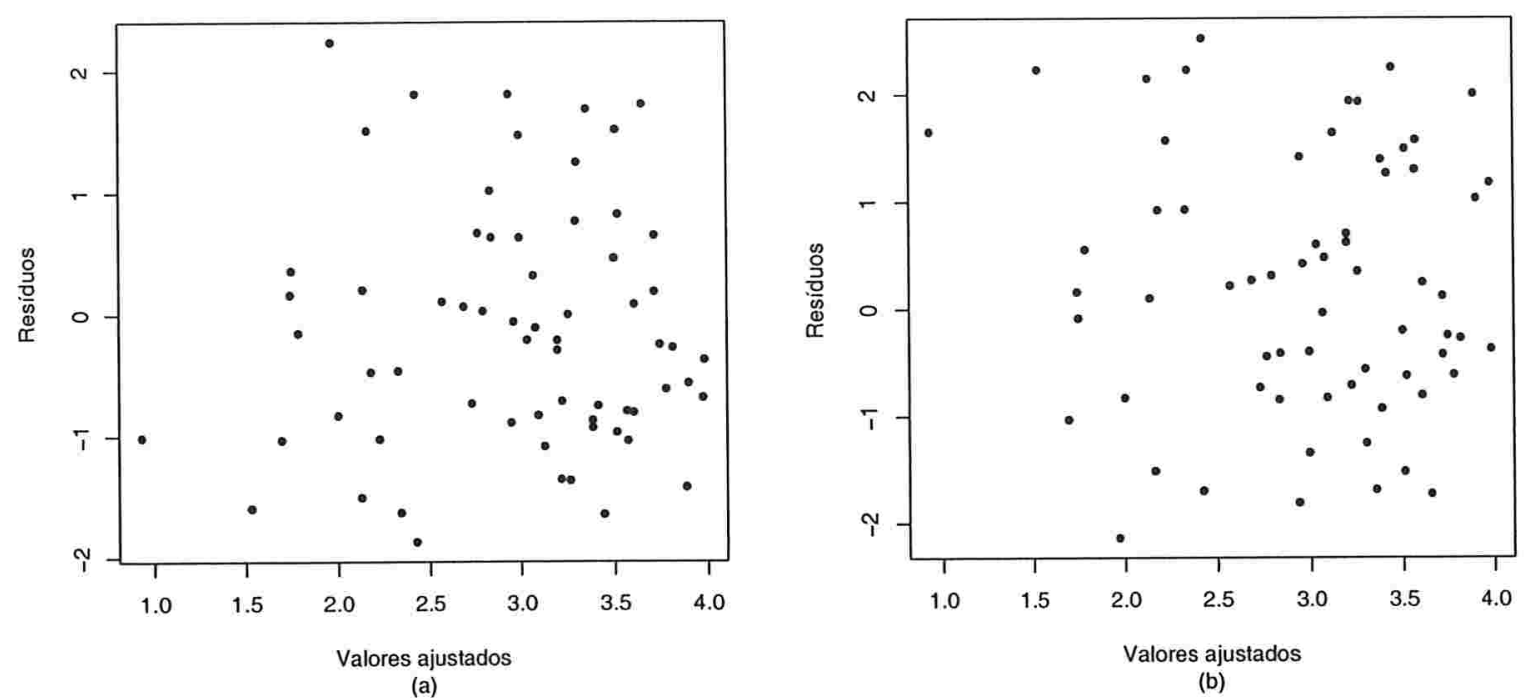

Figura 2.4: gráficos dos resíduos $r_{D C_{i}}^{*}$ (a) e $r_{M D_{i}}^{*}$ (b) contra os valores ajustados.

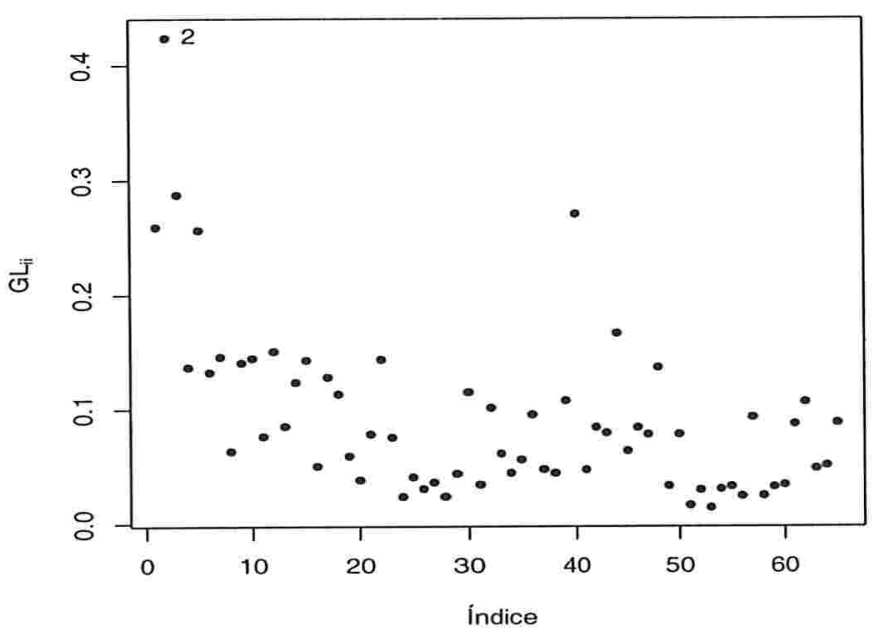

Figura 2.5: gráfico da medida de alavanca generalizada contra o índice das observações.

as observações \#62, \#64 e \#65 como possivelmente influentes (ver Figura 2.11). Fizemos também os gráficos de influência local total $C_{i}$ contra o índice das observações considerando $\widehat{\boldsymbol{\theta}}, \widehat{\boldsymbol{\beta}}$ e $\widehat{\alpha}$ como parâmetros de interesse. Esses gráficos foram omitidos aqui porque destacaram as mesmas observações que os gráficos de $\left|l_{\max }\right|$ contra o índice das observações, confirmando assim uma influência relevante das observações \#3, \#5, \#40, \#44, \#48, \#62, \#64 e \#65. 

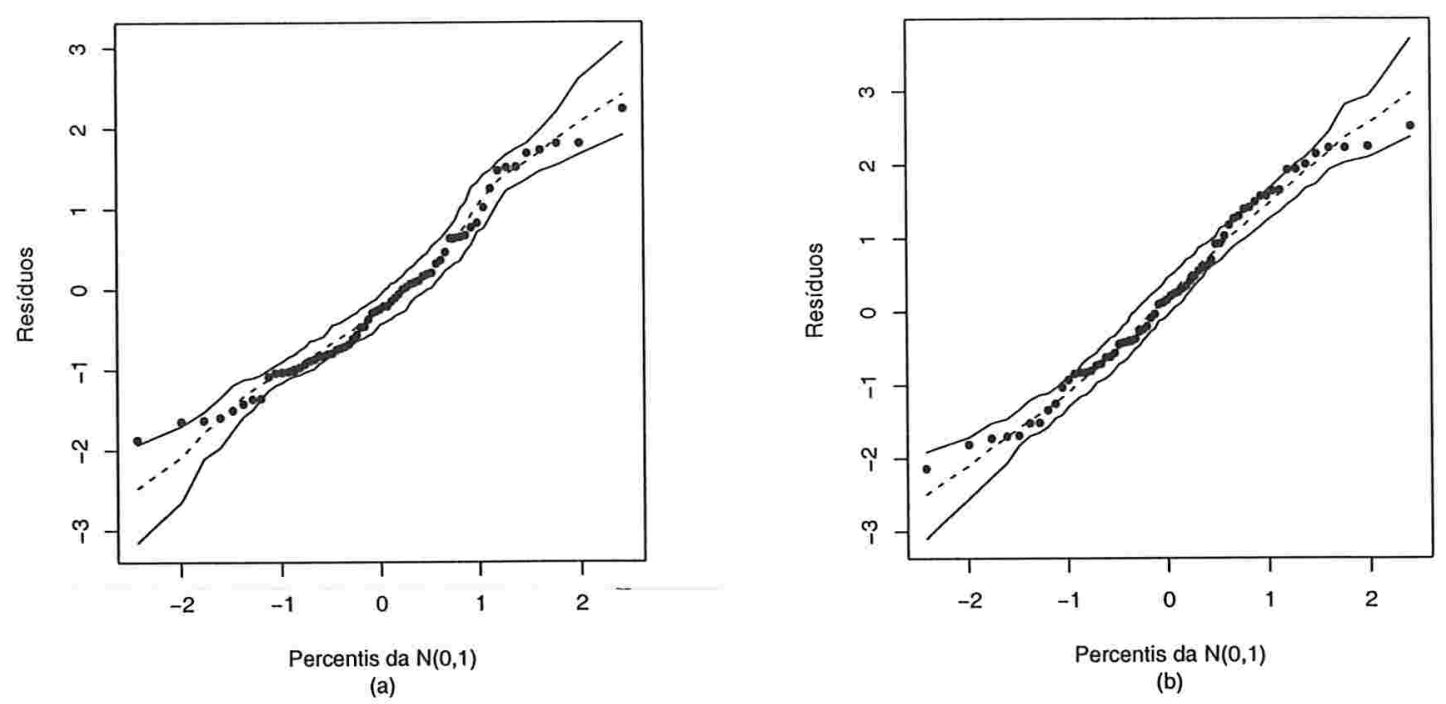

Figura 2.6: gráficos normais de probabilidade para os resíduos $r_{D C_{i}}^{*}$ (a) e $r_{M D_{i}}^{*}$ (b) com envelopes.

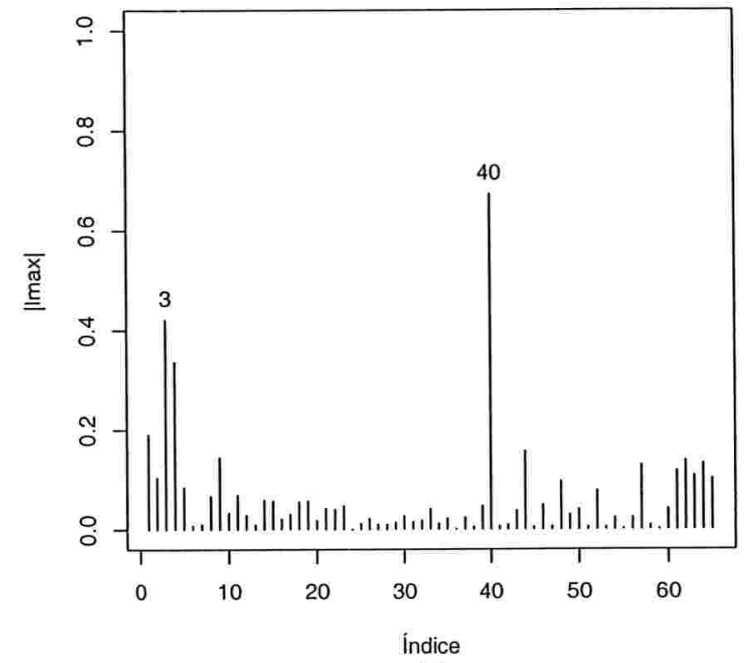

(a)

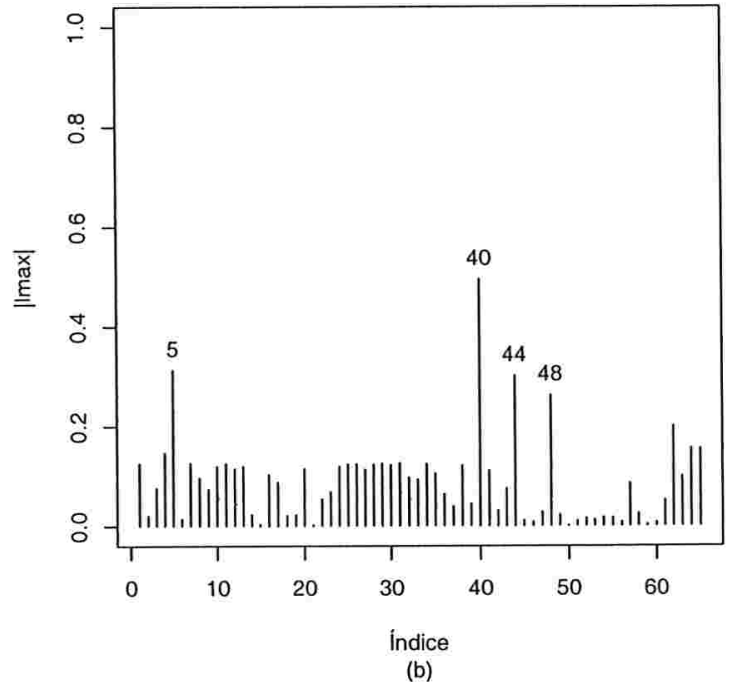

(b)

Figura 2.7: gráficos de $\left|l_{\max }\right|$ contra o índice das observações sob o esquema de perturbação da ponderação de casos: (a) interesse em $\beta$, (b) interesse em $\alpha$. 


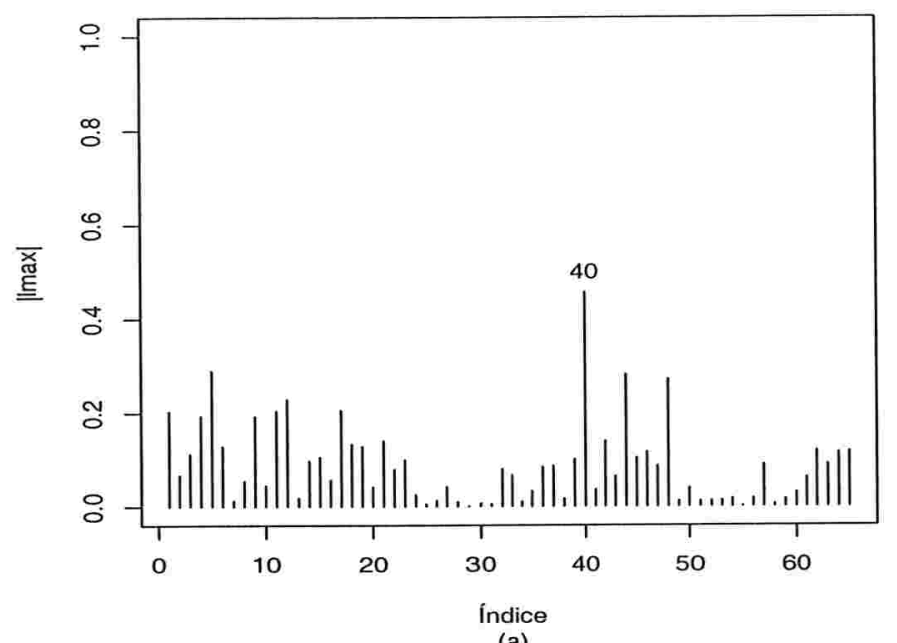

(a)

Figura 2.8: gráfico de $\left|l_{\max }\right|$ para $\theta$ contra o índice das observações sob o esquema de perturbação da variável resposta.

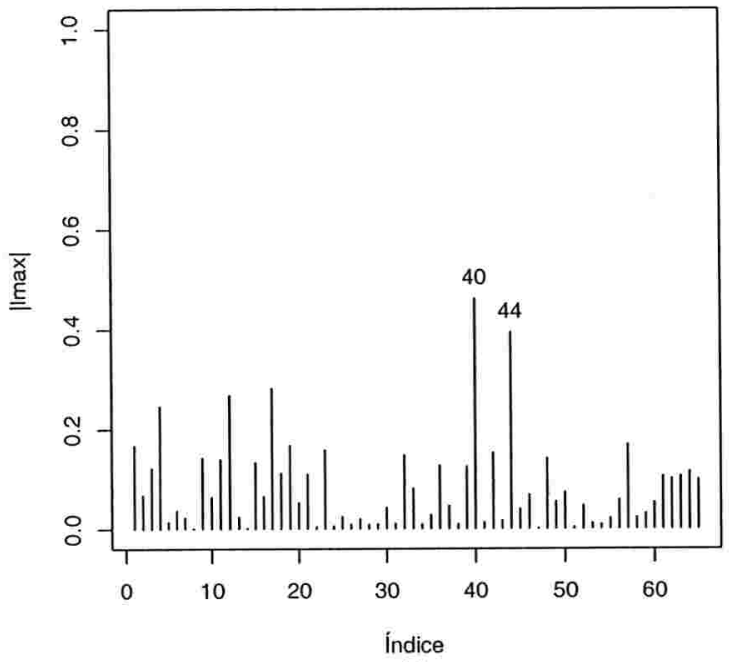

(a)

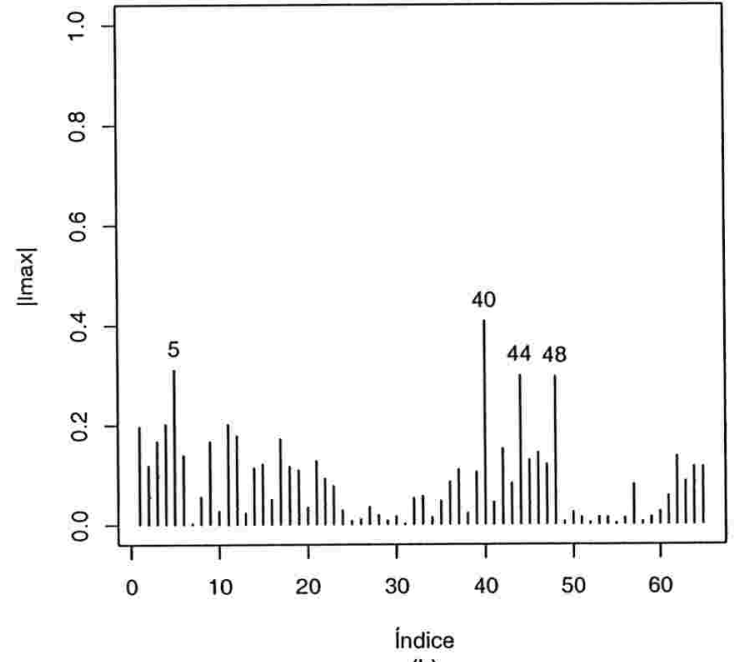

(b)

Figura 2.9: gráficos de $\left|l_{\max }\right|$ contra o índice das observações sob o esquema de perturbação da variável explicativa $x_{2}$ : (a) interesse em $\boldsymbol{\beta}$, (b) interesse em $\alpha$.

Assim, a análise de diagnóstico detectou como potencialmente influentes os seguintes nove casos: \#2, \#3, \#5, \#40, \#44, \#48, \#62, \#64 e \#65. A fim de analisar o impacto dessas observações nas estimativas dos parâmetros realizamos uma análise confirmatória reajustando 


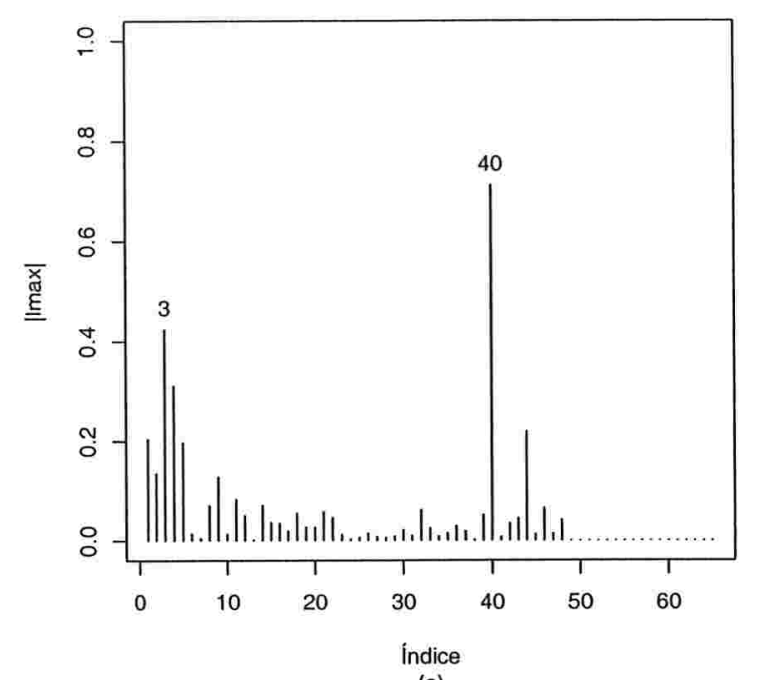

(a)

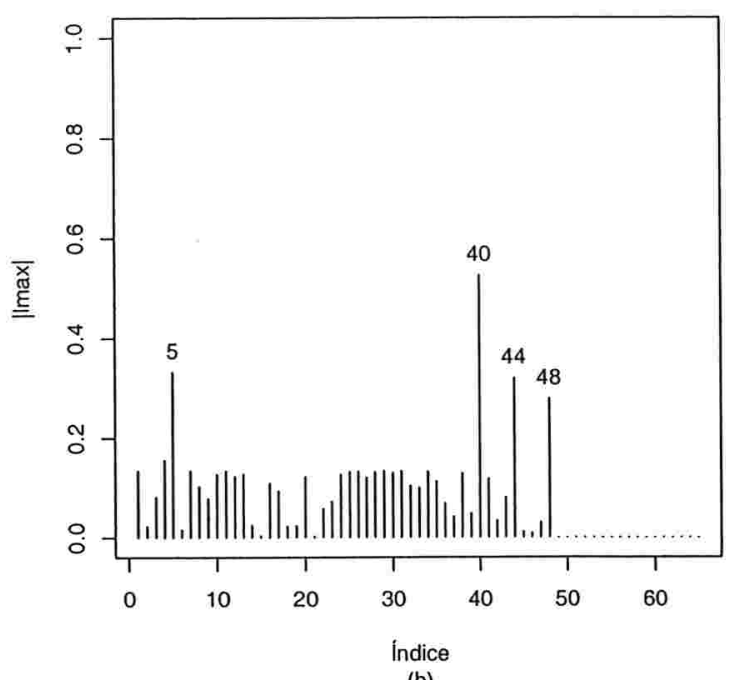

(b)

Figura 2.10: gráficos de $\left|l_{\max }\right|$ contra o índice das observações sob o esquema de perturbação da ponderação de casos não censurados: (a) interesse em $\boldsymbol{\beta}$, (b) interesse em $\alpha$.

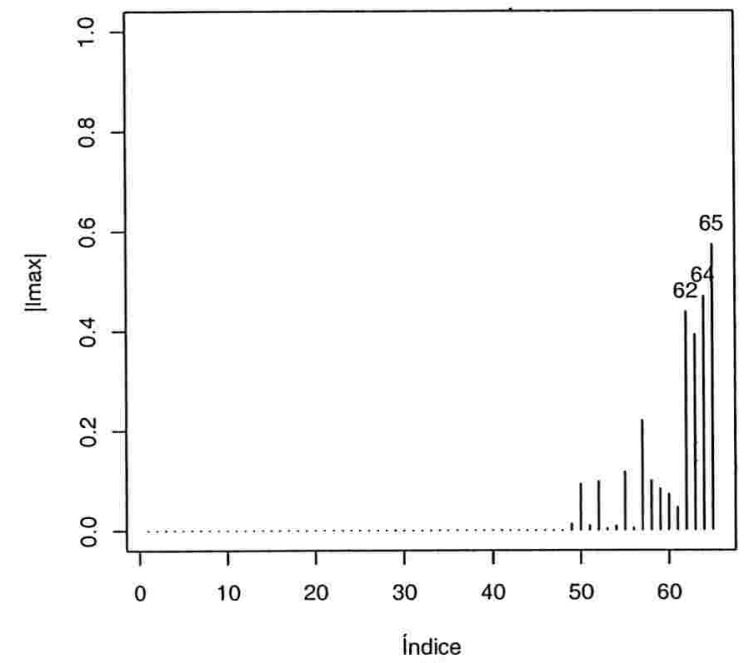

(a)

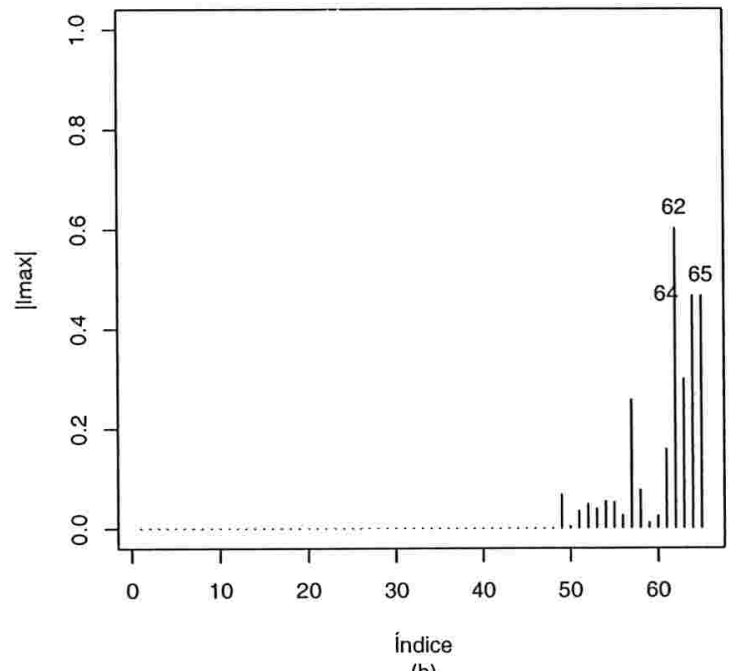

(b)

Figura 2.11: gráficos de $\left|l_{\max }\right|$ contra o índice das observações sob o esquema de perturbação da ponderação de casos censurados: (a) interesse em $\beta$, (b) interesse em $\alpha$. 
o modelo sob algumas situações. Primeiramente, reajustamos o modelo eliminando individualmente cada observação considerada possivelmente influente. Depois, reajustamos o modelo eliminando do "conjunto A" (conjunto original com todas as observações) as nove observações consideradas potencialmente influentes, o qual chamamos de "conjunto B". E ainda reajustamos o modelo removendo o mesmo número de observações consideradas como influentes (nove) escolhidas aleatoriamente entre as observações não classificadas como influentes, que chamamos de "conjunto E". Note que o "conjunto E" possui o mesmo número de observações do "conjunto B" (65-9=56). Ou seja, o "conjunto E" é formado pelas observações do "conjunto A" menos nove observações, selecionadas aleatoriamente entre as observações consideradas não influentes, que neste caso foram \#6, \#14,\#19, \#30, \#33, \#41, \#46, \#54 e \#63.

$\mathrm{Na}$ Tabela 2.1, apresentamos as estimativas dos parâmetros do modelo para cada conjunto A, B e E. Na Tabela 2.2, apresentamos as mudanças relativas, em porcentagem, de cada estimativa, definida por: $\mathrm{RC}_{\theta_{j}}=\left[\left(\widehat{\theta}_{j}-\widehat{\theta}_{j(I)}\right) / \widehat{\theta}_{j}\right] \times 100$, e os níveis descritivos correspondentes, em que $\widehat{\theta}_{j(I)}$ denota a estimativa de máxima verossimilhança de $\theta_{j}$ obtida depois de remover o "conjunto I" de observações.

Seguindo o mesmo procedimento sugerido por Lee, Lu e Song (2006), usamos a mudança relativa total, $\mathrm{TRC}=\sum_{j=0}^{7}\left|\mathrm{RC}_{\theta_{j}}\right|$, a mudança máxima relativa, $\mathrm{MRC}=\max _{j}\left|\mathrm{RC}_{\theta_{j}}\right|$ e a distância entre as verossimilhanças, $\operatorname{LD}_{I}(\boldsymbol{\theta})=2\left\{\ell(\widehat{\boldsymbol{\theta}})-\ell\left(\widehat{\boldsymbol{\theta}}_{(I)}\right)\right\}$, em que $\widehat{\boldsymbol{\theta}}_{(I)}$ denota a estimativa de máxima verossimilhança de $\theta$ obtida depois do "conjunto I" de observações ter sido removido (ver Cook, Peña e Weisberg, 1988). Encontramos para o "conjunto B", $\mathrm{TRC}=273,30, \mathrm{MRC}=148,02 \mathrm{e} \mathrm{LD}_{A-B}(\boldsymbol{\theta})=11,92$; e para o "conjunto E", $\mathrm{TRC}=41,74$, $\mathrm{MRC}=15,84$ e $\operatorname{LD}_{A-E}(\boldsymbol{\theta})=0,19$. Como podemos observar, os resultados são mais sensíveis em relação ao grupo de observações influentes. É válido salientar que o cálculo dessas medidas é apenas ilustrativo pois, a rigor, deveríamos considerar todas as possíveis combinações de nove elementos para formar o "conjunto E", o que seria muito dispendioso computacionalmente. A análise da Tabela 2.1 revela que, fixado o nível de significância em $10 \%$, o resultado inferencial é o mesmo se considerados os conjuntos $\mathrm{A}, \mathrm{B}$ ou E. Isto é, as variavéis explicativas $x_{3}$ e $x_{4}$ permanecem sendo não significativas e, portanto, devem ser retiradas do modelo. Entretanto, analisando a Tabela 2.2, notamos que a eliminação das observações 2 e 3, individualmente, faz com que a variável explicativa $x_{5}$ deixe de ser significativa. Percebemos, assim, que a significância dessa variável foi mascarada por essas observações e, portanto, essa variável deve também ser removida do modelo. Desta forma, chegamos ao modelo final dado por

$$
y_{i}=\beta_{0}+\beta_{1} x_{i 1}+\beta_{2} x_{i 2}+\varepsilon_{i}, i=1, \ldots, 65
$$

As estimativas de máxima verossimilhança dos parâmetros (erro padrão aproximado entre 
parênteses) são

$$
\widehat{\beta}_{0}=4,409(0,772), \widehat{\beta}_{1}=-1,869(0,423), \widehat{\beta}_{2}=0,109(0,049) \text { e } \widehat{\alpha}=1,140(0,118) \text {. }
$$

A interpretação dos coeficientes estimados do modelo final pode ser feita como segue. O tempo de sobrevivência esperado decresce aproximadamente $85 \%\left[\left(1-e^{-1,869}\right) \times 100 \%\right]$ quando a taxa de uréia nitrogenada do sangue (em logaritmo) cresce em uma unidade, permanecendo fixa a taxa de hemoglobina. Similarmente, o tempo de sobrevivência esperado cresce aproximadamente $12 \%\left[e^{0,109} \times 100 \%\right]$ quando a taxa de hemoglobina cresce em uma unidade enquanto a taxa de uréia nitrogenada do sangue (em logaritmo) é mantida fixa.

Tabela 2.1: estimativas dos parâmetros e os níveis descritivos $(p)$ para o conjunto indicado.

\begin{tabular}{cccccccc}
\hline Conjunto & Parâmetro & $\beta_{0}$ & $\beta_{1}$ & $\beta_{2}$ & $\beta_{3}$ & $\beta_{4}$ & $\beta_{5}$ \\
\hline A & Estimativa & 4,500 & $-1,596$ & 0,142 & 0,010 & 0,209 & $-0,141$ \\
& $p$ & 0,000 & 0,000 & 0,004 & 0,455 & 0,461 & 0,042 \\
\hline \multirow{2}{*}{$\mathrm{B}$} & Estimativa & 5,685 & $-1,617$ & 0,099 & $-0,005$ & 0,119 & $-0,142$ \\
& $p$ & 0,000 & 0,000 & 0,037 & 0,692 & 0,634 & 0,105 \\
\hline E & Estimativa & 4,598 & $-1,580$ & 0,129 & 0,009 & 0,243 & $-0,135$ \\
& $p$ & 0,001 & 0,001 & 0,020 & 0,521 & 0,435 & 0,067 \\
\hline
\end{tabular}

Estudo dos tempos de sobrevivência de pacientes com câncer de pulmão

Para esta aplicação consideramos o conjunto de dados apresentado em Kalbfleisch e Prentice (2002, p. 378), onde homens com câncer de pulmão avançado foram aleatorizados entre um tratamento de quimioterapia padrão e um teste. O objetivo do estudo é tentar explicar o tempo de sobrevivência ( $\mathrm{T}$, em dias) de 137 pacientes com câncer de pulmão (nove deles censurados) através de uma estrutura de regressão com as seguintes variáveis explicativas: uma medida de aleatorização do estado do paciente (Karnofsky) $\left(x_{1}\right)$ : 10 - 30 completamente hospitalizado, 40 - 60 parcialmente confinado, 70 - 90 capacidade do paciente de cuidar-se sozinho; tempo em meses do diagnóstico até a aleatorização $\left(x_{2}\right)$; idade em anos, no instante da aleatorização $\left(x_{3}\right)$; tratamento anterior $\left(x_{4}\right)$, dicotomizada em 10 para sim e 0 para não; tipo histológico do tumor: squamous, small cell, adeno, large cell e tipo de tratamento $\left(x_{8}\right)$ : 0 para padrão e 1 para teste. Para os tipos histológicos de tumor consideramos três variáveis auxiliares $x_{5}=1, x_{6}=1$ e $x_{7}=1$ se o tipo de célula do câncer é squamous, small cell e adeno, respectivamente, e 0 caso contrário. 
Tabela 2.2: mudanças relativas $(\mathrm{RC}$, em \%) e os correspondentes níveis descritivos entre parênteses.

\begin{tabular}{cccccccc}
\hline Conjunto $(I)$ & $\widehat{\beta}_{0}$ & $\widehat{\beta}_{1}$ & $\widehat{\beta}_{2}$ & $\widehat{\beta}_{3}$ & $\widehat{\beta}_{4}$ & $\widehat{\beta}_{5}$ & $\widehat{\alpha}$ \\
\hline A-\{observação 2\} & 4 & 6 & 1 & 54 & 3 & 27 & 0 \\
& $(0,001)$ & $(0,001)$ & $(0,005)$ & $(0,753)$ & $(0,473)$ & $(0,201)$ & \\
A-\{observação 3\} & 15 & -3 & 3 & -62 & -4 & 30 & 1 \\
& $(0,005)$ & $(0,000)$ & $(0,005)$ & $(0,250)$ & $(0,438)$ & $(0,188)$ & \\
A-\{observação 5 $\}$ & -20 & -3 & 25 & 42 & 68 & -9 & 4 \\
& $(0,000)$ & $(0,000)$ & $(0,046)$ & $(0,660)$ & $(0,814)$ & $(0,023)$ & \\
A-\{observação 40\} & -14 & 0 & -14 & 71 & -12 & -34 & 6 \\
& $(0,000)$ & $(0,000)$ & $(0,000)$ & $(0,826)$ & $(0,377)$ & $(0,011)$ & \\
A-\{observação 44\} & 12 & -1 & -14 & -22 & -46 & 12 & 3 \\
& $(0,002)$ & $(0,000)$ & $(0,001)$ & $(0,350)$ & $(0,283)$ & $(0,063)$ & \\
A-\{observação 48\} & -5 & -2 & 5 & 4 & 52 & -7 & 3 \\
& $(0,000)$ & $(0,000)$ & $(0,006)$ & $(0,460)$ & $(0,723)$ & $(0,029)$ & \\
A-\{observação 62\} & -5 & -6 & 9 & 23 & -7 & 11 & 2 \\
& $(0,000)$ & $(0,000)$ & $(0,009)$ & $(0,562)$ & $(0,422)$ & $(0,065)$ & \\
A-\{observação 64\} & -2 & 3 & 5 & 14 & -9 & -1 & 1 \\
& $(0,000)$ & $(0,000)$ & $(0,006)$ & $(0,513)$ & $(0,415)$ & $(0,037)$ & \\
A-\{observação 65\} & -2 & 6 & 8 & 5 & -9 & -6 & 1 \\
& $(0,000)$ & $(0,000)$ & $(0,008)$ & $(0,471)$ & $(0,414)$ & $(0,031)$ & \\
B & -26 & -1 & 30 & 148 & 43 & -1 & 24 \\
& $(0,000)$ & $(0,000)$ & $(0,037)$ & $(0,692)$ & $(0,634)$ & $(0,105)$ & \\
E & -2 & 1 & 9 & 7 & -16 & 4 & -3 \\
& $(0,001)$ & $(0,001)$ & $(0,020)$ & $(0,521)$ & $(0,435)$ & $(0,067)$ & \\
\hline
\end{tabular}

Este conjunto de dados tem sido analisado por meio de modelos de regressão usando diferentes distribuições para os erros, a saber: exponencial, gama generalizada, log-logística, log-normal e Weibull (veja, por exemplo, Lee e Wang, 2003).

Baseado no argumento de tipo de fadiga, dado na aplicação anterior, vamos ajustar esses dados assumindo, inicialmente, o modelo de regressão linear log-BS para dados censurados, dado em (2.1). Especificamente, este modelo é dado por

$$
y_{i}=\beta_{0}+\beta_{1} x_{i 1}+\cdots+\beta_{8} x_{i 8}+\varepsilon_{i}, i=1, \ldots, 137,
$$

em que $\varepsilon_{i}$ são variáveis aleatórias independentes e identicamente distribuídas tais que $\varepsilon_{i} \sim$ $S N(\alpha, 0,2)$ e a resposta $y_{i}=\log \left(t_{i}\right)$ denota o logaritmo do tempo de sobrevivência ou do tempo de censura. Uma vez que $\mathbb{E}\left(\varepsilon_{i}\right)=0$, a regressão é feita sob $\mathbb{E}\left\{\log \left(T_{i}\right)\right\}$. As estimativas de máxima verossimilhança de $\boldsymbol{\theta}=\left(\boldsymbol{\beta}^{\top}, \alpha\right)^{\top}=\left(\beta_{0}, \beta_{1}, \ldots, \beta_{8}, \alpha\right)^{\top}$ são (erro padrão aproximado entre parênteses):

$$
\begin{gathered}
\widehat{\beta}_{0}=1,142(0,638), \widehat{\beta}_{1}=0,040(0,005), \widehat{\beta}_{2}=-0,003(0,009), \widehat{\beta}_{3}=0,022(0,008), \\
\widehat{\beta}_{4}=-0,002(0,023), \widehat{\beta}_{5}=-0,2800(0,303), \widehat{\beta}_{6}=-0,705(0,301),
\end{gathered}
$$




$$
\widehat{\beta}_{7}=-0,691(0,367), \widehat{\beta}_{8}=0,383(0,193) \text { e } \widehat{\alpha}=1,262(0,079) .
$$

Fixado o nível de significância em $5 \%$, temos que as variáveis explicativas $x_{2}, x_{4}, x_{5}, x_{7}$ e $x_{8}$ são marginalmente não significativas. Ou seja, esta análise inicial indicaria que não há dependência aparente dos tempos de sobrevivência com a duração da doença até a entrada no estudo clínico, bem como se houve ou não tratamento anterior, e com o tipo de quimioterapia, se é padrão ou teste.

A análise de diagnóstico baseada nos resíduos componente do desvio e tipo martingale, mostrada na Figura 2.12 revela um forte destaque para a observação \#85. Essa observação corresponde a um paciente com 35 anos de idade que apresenta tempo de sobrevivência de apenas um dia e esperou sete meses do diagnóstico até a aleatorização. Ele esteve parcialmente confinado, não fez tratamento anterior, seu tipo histológico de tumor foi squamous e o tratamento recebido foi o teste. Os gráficos normais de probabilidade com envelopes gerados para os resíduos componente do desvio e tipo martingale são apresentados na Figura 2.13 e mostram uma indicação de que uma distribuição com caudas mais pesadas para os erros seria mais apropriada. Além disso, três observações não pertencem ao envelope gerado, correspondentes aos pacientes \#77, \#85 e \#100 apontados na Figura 2.12. Os gráficos de influência local e influência local total sob o esquema de perturbação da ponderação de casos são mostrados nas Figuras 2.14 e 2.15, respectivamente. Mais uma vez, usando o ponto de corte proposto por Verbeke e Molenbergs (2000, Seção 11.3), dado em (2.9), nos gráficos de influência local total, as três observações mencionadas acima são destacadas como influentes.

A Tabela 2.3 mostra as variações percentuais nas estimativas dos parâmetros do modelo quando eliminamos, individualmente, as observações consideradas como influentes e quando eliminamos conjuntamente essas observações (representada pelo conjunto $I=\{77,85$ e 100 $\}$ ). Os níveis descritivos para as novas estimativas são dados entre parênteses. Como podemos observar, em geral, as variações percentuais são grandes, mesmo para as variáveis que foram significativas a um nível de 5\%. Adicionalmente, notamos que há mudança inferencial para alguns coeficientes. Em particular, $\beta_{3}$ e $\beta_{8}$ deixam de ser significativos a um nível de $5 \%$ quando eliminamos as três observações consideradas influentes.

Embora seja possível escolhermos um modelo final com o exposto acima, notamos que as estimativas de máxima verossimilhança apresentam falta de robustez contra observações extremas e a suposição para a distribuição dos erros não parece ser adequada. Analisaremos novamente esses dados no Capítulo 4 sob um modelo cuja distribuição para os erros possui caudas pesadas. 


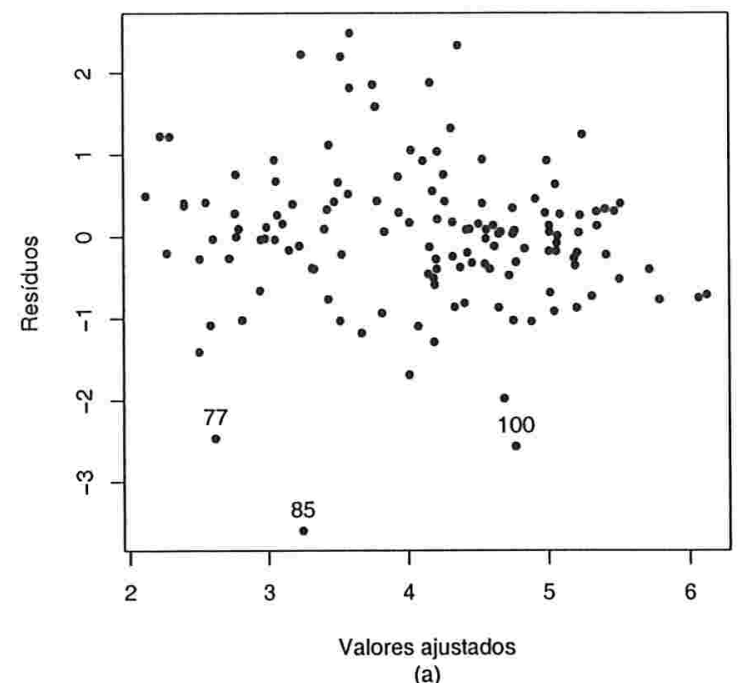

(a)

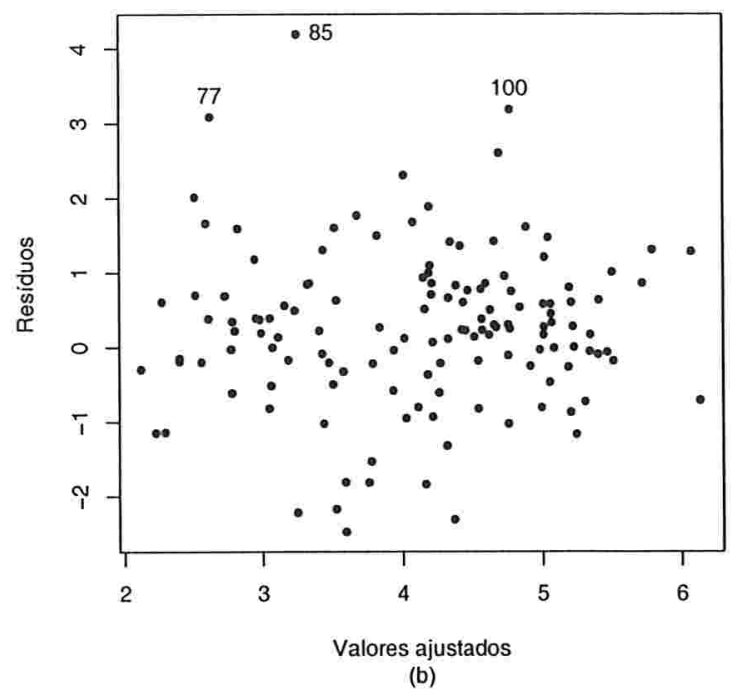

Figura 2.12: gráficos dos resíduos $r_{D C_{i}}$ (a) e $r_{M D_{i}}$ (b) contra os valores ajustados.

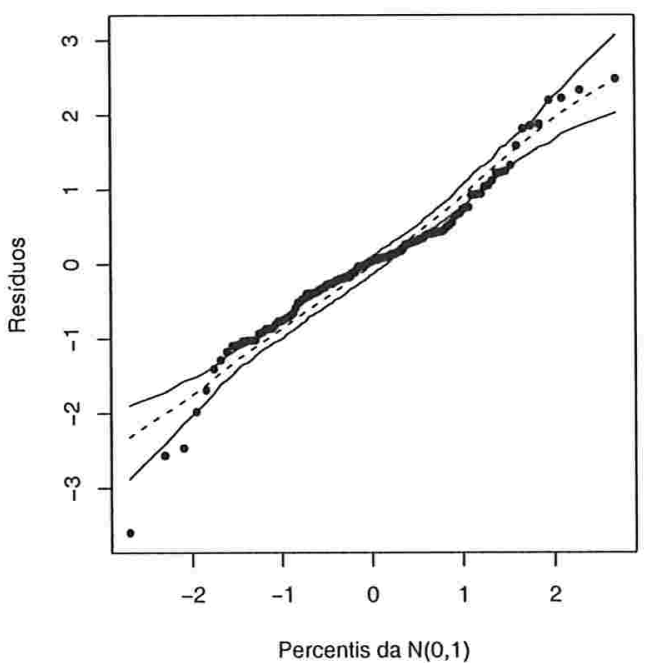

(a)

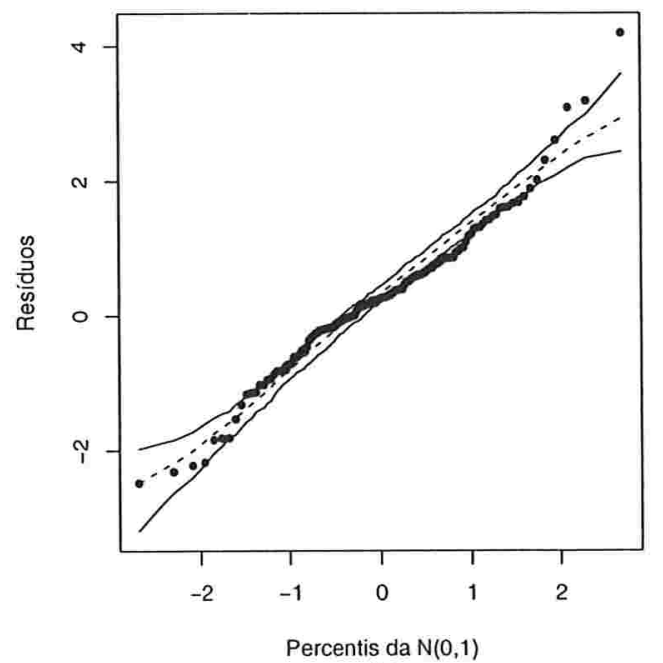

(b)

Figura 2.13: gráficos normais de probabilidade para os resíduos $r_{D C_{i}}$ (a) e $r_{M D_{i}}$ (b) com envelopes gerados. 


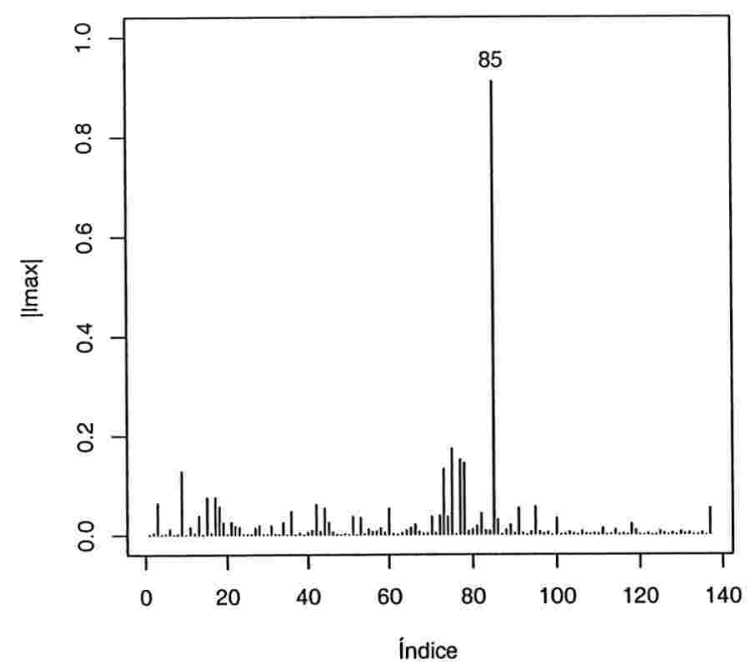

(a)

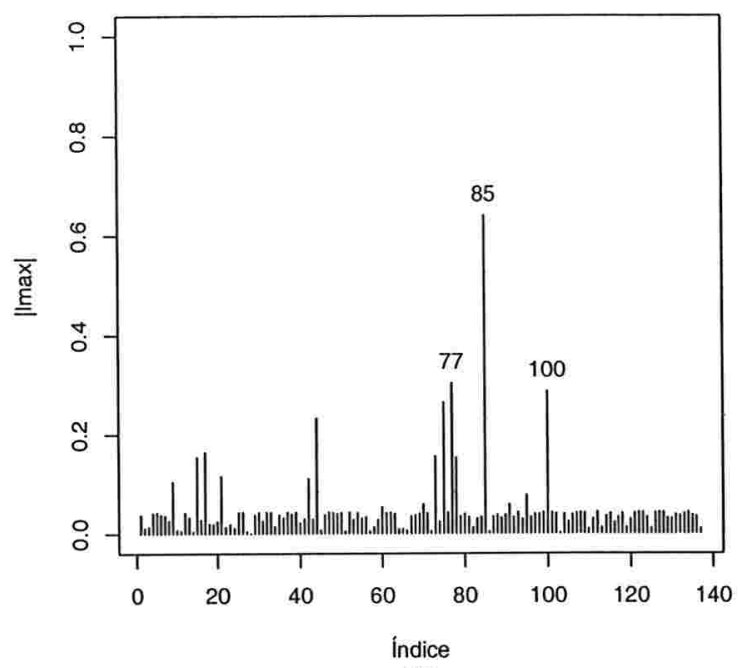

(b)

Figura 2.14: gráficos de $\left|l_{\max }\right|$ contra o índice das observações sob o esquema de perturbação da ponderação de casos: (a) interesse em $\boldsymbol{\beta}$, (b) interesse em $\alpha$.

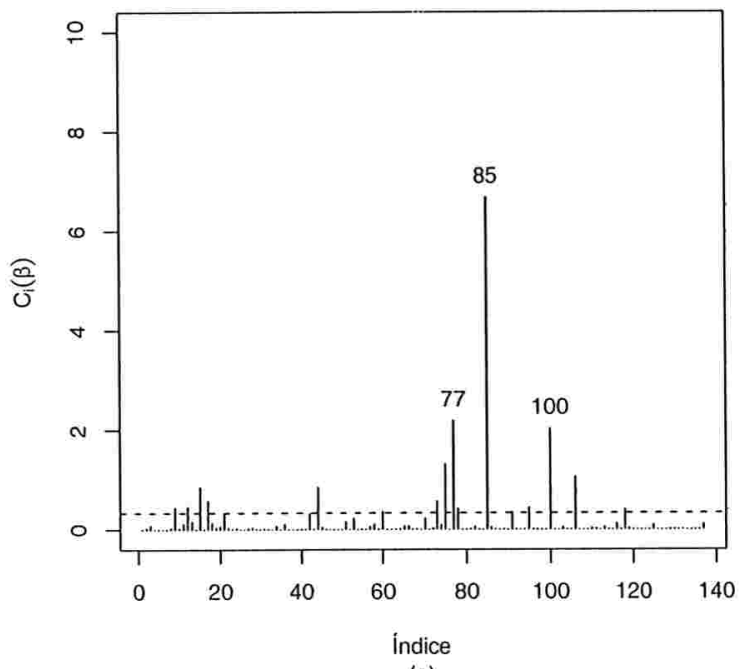

(a)

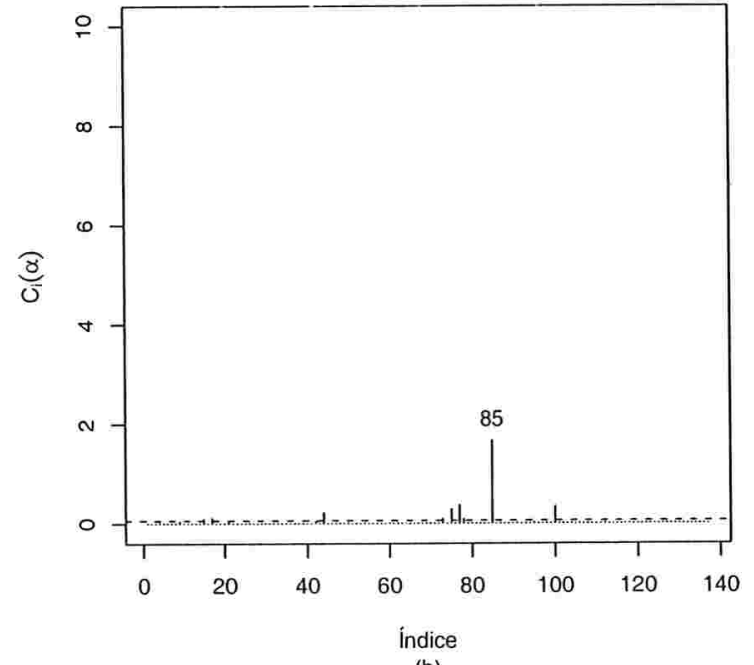

(b)

Figura 2.15: gráficos de $C_{i}(\boldsymbol{\beta})$ (a) e $C_{i}(\alpha)$ (b) contra o índice das observações sob o esquema de perturbação da ponderação de casos. 
Tabela 2.3: mudanças relativas $(-\mathrm{RC}-\mathrm{em} \%)$ e os correspondentes níveis descritivos entre parênteses.

\begin{tabular}{cccccc}
\hline & \multicolumn{5}{c}{ Observação eliminada } \\
Estimativa dos coeficientes & Nenhuma & 77 & 85 & 100 & Conjunto I \\
\hline$\widehat{\beta}_{0}$ & - & -3 & -104 & 35 & -81 \\
& $(0,073)$ & $(0,053)$ & $(0,000)$ & $(0,243)$ & $(0,000)$ \\
$\widehat{\beta}_{1}$ & - & 11 & 8 & -8 & 14 \\
& $(0,000)$ & $(0,000)$ & $(0,000)$ & $(0,000)$ & $(0,000)$ \\
$\widehat{\beta}_{2}$ & - & -37 & -15 & -40 & -108 \\
& $(0,750)$ & $(0,659)$ & $(0,688)$ & $(0,651)$ & $(0,439)$ \\
$\widehat{\beta}_{3}$ & - & -16 & 77 & -14 & 51 \\
& $(0,006)$ & $(0,001)$ & $(0,549)$ & $(0,001)$ & $(0,158)$ \\
$\widehat{\beta}_{4}$ & - & 556 & -1261 & -155 & -756 \\
& $(0,927)$ & $(0,673)$ & $(0,200)$ & $(0,810)$ & $(0,382)$ \\
$\widehat{\beta}_{5}$ & - & 28 & 77 & -2 & 119 \\
& $(0,356)$ & $(0,489)$ & $(0,817)$ & $(0,361)$ & $(0,833)$ \\
$\widehat{\beta}_{6}$ & - & -4 & -2 & 20 & 11 \\
& $(0,019)$ & $(0,011)$ & $(0,008)$ & $(0,053)$ & $(0,011)$ \\
$\widehat{\beta}_{7}$ & - & -9 & -17 & -4 & -28 \\
& $(0,060)$ & $(0,039)$ & $(0,014)$ & $(0,042)$ & $(0,002)$ \\
$\widehat{\beta}_{8}$ & - & 18 & 48 & 36 & 101 \\
& $(0,047)$ & $(0,092)$ & $(0,268)$ & $(0,210)$ & $(0,981)$ \\
$\widehat{\alpha}$ & - & 3 & 9 & 4 & 17 \\
\hline & & & & &
\end{tabular}




\section{Capítulo 3}

\section{Distribuição Birnbaum-Saunders Generalizada}

Nas últimas décadas uma família de distribuições multivariadas cujos contornos de suas densidades têm a mesma forma elíptica que a distribuição normal tem sido bastante estudada. Em particular, essa classe inclui distribuições com caudas mais ou menos pesadas do que a distribuição normal, além de incluir essa última como caso particular. Essa família é denominada distribuições de contornos elípticos ou simplesmente elípticas (veja, por exemplo, Díaz-García, Leiva e Galea, 2002). As distribuições elípticas têm sido estudadas por vários autores, entre eles Fang e Anderson (1990), Fang e Zhang (1990), Fang, Kotz e Ng (1990), Gupta e Varga (1993) e Arellano-Valle (1994).

Para uma variável aleatória (caso unidimensional), as distribuições elípticas correspondem a todas as distribuições simétricas em $\mathbb{R}$. Assim, uma variável aléatória $X$ segue uma distribuição elíptica se sua função característica é dada por

$$
\psi(t)=\exp (i t \mu) \phi\left(t^{2} \sigma^{2}\right)
$$

$\operatorname{com} \phi: \mathbb{R}^{+} \rightarrow \mathbb{R}$, ou se a fdp de $X$ é dada por

$$
f_{X}(x)=c g\left[\frac{(x-\mu)^{2}}{\sigma^{2}}\right]=c g(u) ; x \in \mathbb{R}, u>0,
$$

em que $g(u)$ é o núcleo da fdp de $X$ e $c$ é a constante normalizadora que faz com que $f_{X}(x)$ seja uma função densidade. Denotamos por $X \sim E C\left(\mu, \sigma^{2} ; \phi\right)$ ou $X \sim E C\left(\mu, \sigma^{2} ; g\right)$ de acordo com (3.1) ou (3.2), respectivamente. Em geral, $\mu$ é o parâmetro de posição e coincide com a média, $\mathbb{E}(X)$, se o primeiro momento da distribuição existe e $\sigma^{2}$ é o parâmetro de escala. Além disso, se os dois primeiros momentos da distribuição existem, $\operatorname{Var}(X)=c_{0} \sigma^{2}$, com $c_{0}=-2 \phi^{\prime}(0)$ e $\phi^{\prime}$ sendo a derivada de $\phi$ dada em (3.1). Como exemplo de distribuições pertencentes a essa 
família podemos citar as distribuições Pearson VII, $t$ de Student, Kotz, Bessel, caso especial, logística, entre outras (ver, por exemplo, Cysneiros, 2004 ou Díaz-García e Leiva, 2005 e 2006).

Díaz-García e Leiva $(2005,2006)$ apresentam uma generalização da distribuição Birnbaum-Saunders, obtendo-a a partir de distribuições de contornos elípticos, a qual denominaram distribuição Birnbaum-Saunders generalizada.

Neste capítulo, apresentaremos, inicialmente, a distribuição BSG proposta por DíazGarcía e Leiva $(2005,2006)$. Em seguida, descreveremos os momentos dessa distribuição e apresentaremos alguns aspectos não discutidos em Díaz-García e Leiva $(2005,2006)$. Os momentos serão usados para obter a média, a variância, os coeficientes de variação, curtose e assimetria. Em seguida, discutiremos a estimação dos parâmetros da distribuição, utilizando os métodos de máxima verossimilhança e dos momentos modificados, proposto por $\mathrm{Ng}$, Kundu e Balakrishnan (2003), assim como resultados de simulações referentes ao processo de estimação. Apresentaremos, também, uma proposta de correção de viés através do método bootstrap.

\subsection{Definição e algumas propriedades da distribuição BSG}

O novo modelo de sobrevivência desenvolvido por Díaz-García e Leiva $(2005,2006)$ é definido em termos das distribuições elípticas. Tal modelo é uma generalização da distribuição Birnbaum-Saunders clássica e foi obtido a partir de (1.4) relaxando a suposição de que $Z \sim$ $N(0,1)$. Ou seja, os autores consideraram

$$
T=\beta\left[\frac{\alpha}{2} U+\sqrt{\left(\frac{\alpha U}{2}\right)^{2}+1}\right]^{2},
$$

em que $U \sim E C(0,1 ; g)$. A nova variável aleatória $T$ segue uma distribuição BirnbaumSaunders generalizada, denotada por $T \sim B S G(\alpha, \beta ; g)$.

Os parâmetros $\alpha$ e $\beta$ são, respectivamente, os parâmetros de forma e escala e $g$ é dada em (3.2). Desta maneira, os autores obtiveram uma nova e mais geral classe de distribuições, que é mais flexível por conter distribuições que possuem caudas mais ou menos pesadas que a distribuição Birnbaum-Saunders clássica e distribuições bimodais (ver Figuras 3.1-3.4).

A função densidade de probabilidade de $T \sim B S G(\alpha, \beta ; g)$ é dada por

$$
f_{T}(t)=\frac{c}{2 \alpha \beta^{1 / 2}} t^{-3 / 2}(t+\beta) g\left(\frac{1}{\alpha^{2}}\left[\frac{t}{\beta}+\frac{\beta}{t}-2\right]\right), t>0
$$


em que $c$ e $g$ são dadas em (3.2). Definindo

$$
a_{t}(\alpha, \beta)=\frac{1}{\alpha}\left(\sqrt{\frac{t}{\beta}}-\sqrt{\frac{\beta}{t}}\right),
$$

temos que

$$
\frac{d}{d t} a_{t}(\alpha, \beta)=\frac{t^{-3 / 2}(t+\beta)}{2 \alpha \beta^{1 / 2}},
$$

e então podemos reescrever (3.4) da seguinte forma:

$$
f_{T}(t)=f\left(a_{t}(\alpha, \beta)\right) \frac{d}{d t} a_{t}(\alpha, \beta),
$$

em que $f$ é dada em (3.2). Vários exemplos para a função $f_{T}$ são considerados em Díaz-García e Leiva $(2005,2006)$, sendo que alguns estão resumidos na Tabela 3.1, onde são apresentadas expressões explícitas para as funções de densidade de uma distribuição BSG obtidas a partir de distribuições elípticas indicadas.

Tabela 3.1: exemplos de distribuições na classe Birnbaum-Saunders generalizada.

\begin{tabular}{cc}
\hline Distribuição & fdp BSG \\
\hline Normal & $\frac{t^{-3 / 2}(t+\beta)}{2 \alpha(2 \pi \beta)^{1 / 2}} \exp \left(-\frac{1}{2 \alpha^{2}}\left[\frac{t}{\beta}+\frac{\beta}{t}-2\right]\right) \alpha, \beta>0$ \\
Pearson VII & $\frac{\Gamma(q) t^{-3 / 2}(t+\beta)}{2 \alpha(r \pi \beta)^{1 / 2} \Gamma\left(q-\frac{1}{2}\right)}\left(1+\frac{1}{r \alpha^{2}}\left[\frac{t}{\beta}+\frac{\beta}{t}-2\right]\right)^{-q} \alpha, \beta, r>0, q>1 / 2$ \\
$t$ de Student & $\frac{\Gamma((\nu+1) / 2) t^{-3 / 2}(t+\beta)}{2 \alpha(\nu \pi \beta)^{1 / 2} \Gamma(\nu / 2)}\left(1+\frac{1}{\nu \alpha^{2}}\left[\frac{t}{\beta}+\frac{\beta}{t}-2\right]\right)^{-(\nu+1) / 2} \alpha, \beta, \nu>0$ \\
Cauchy & $\frac{t^{-3 / 2}(t+\beta)}{2 \alpha(\pi \beta)^{1 / 2}}\left(1+\frac{1}{\alpha^{2}}\left[\frac{t}{\beta}+\frac{\beta}{t}-2\right]\right)^{-1} \alpha, \beta>0$ \\
Caso especial & $\frac{t^{-3 / 2}(t+\beta)}{\pi \alpha(2 \beta)^{1 / 2}}\left(1+\frac{1}{\alpha^{4}}\left[\frac{t}{\beta}+\frac{\beta}{t}-2\right]^{2}\right)^{-1} \alpha, \beta>0$ \\
Kotz & $\frac{s r^{(2 q-1) /(2 s)} t^{-3 / 2}(t+\beta)}{2 \alpha^{2 q-1} \beta^{1 / 2} \Gamma(2 q-1 / 2 s)}\left(\frac{t}{\beta}+\frac{\beta}{t}-2\right)^{q-1} \exp \left(-\frac{r}{\alpha^{2 s}}\left[\frac{t}{\beta}+\frac{\beta}{t}-2\right]^{s}\right) \alpha, \beta, r, s>0, q>1 / 2$ \\
Logística & $\frac{t^{-3 / 2}(t+\beta)}{\left(\int_{0}^{\infty} \frac{z^{-1 / 2} \operatorname{cxp}(-z)}{(1+\exp (-z))^{2}} d z\right) 2 \alpha \beta \beta^{1 / 2}} \frac{\exp \left(-\frac{1}{\alpha^{2}}\left[\frac{t}{\beta}+\frac{\beta}{t}-2\right]\right)}{\left(1+\exp \left(-\frac{1}{\alpha^{2}}\left[\frac{t}{\beta}+\frac{\beta}{t}-2\right]\right)\right)^{2}} \alpha, \beta>0$ \\
\hline
\end{tabular}

A seguir apresentaremos alguns gráficos da distribuição BSG obtida a partir de distribuições elípticas indicadas. Observando a Figura 3.1 temos que, quando o parâmetro $r$ 
cresce, a distribuição Birnbaum-Saunders gerada pela distribuição Pearson VII tende a ser mais platicúrtica e assimétrica, o parâmetro $r$ modifica então a forma e a escala da distribuição. Por outro lado, quando o parâmetro $q$ cresce, a curtose decresce, a forma e a escala não se alteram. Na Figura 3.2 percebemos que, quando o parâmetro $\nu$ cresce, a distribuição tende à distribuição Birnbaum-Saunders clássica, ou seja, gerada pela distribuição normal. Além disso, encontramos distribuições com comportamentos bem distintos geradas pelas distribuições logística, caso especial e Laplace. A Figura 3.3 ilustra uma distribuição que não possui momentos, gerada pela distribuição Cauchy. Observa-se, também, que as distribuições Cauchy e Kotz são bimodais. Na Figura 3.4 tem-se densidades geradas pela distribuição Kotz. Tem-se que, quando o parâmetro $r$ cresce, a distribuição tende a ser mais leptocúrtica. $\mathrm{O}$ parâmetro $q$ modifica a escala inversamente e o parâmetro $s$ está afetando diretamente a separação entre as duas modas.
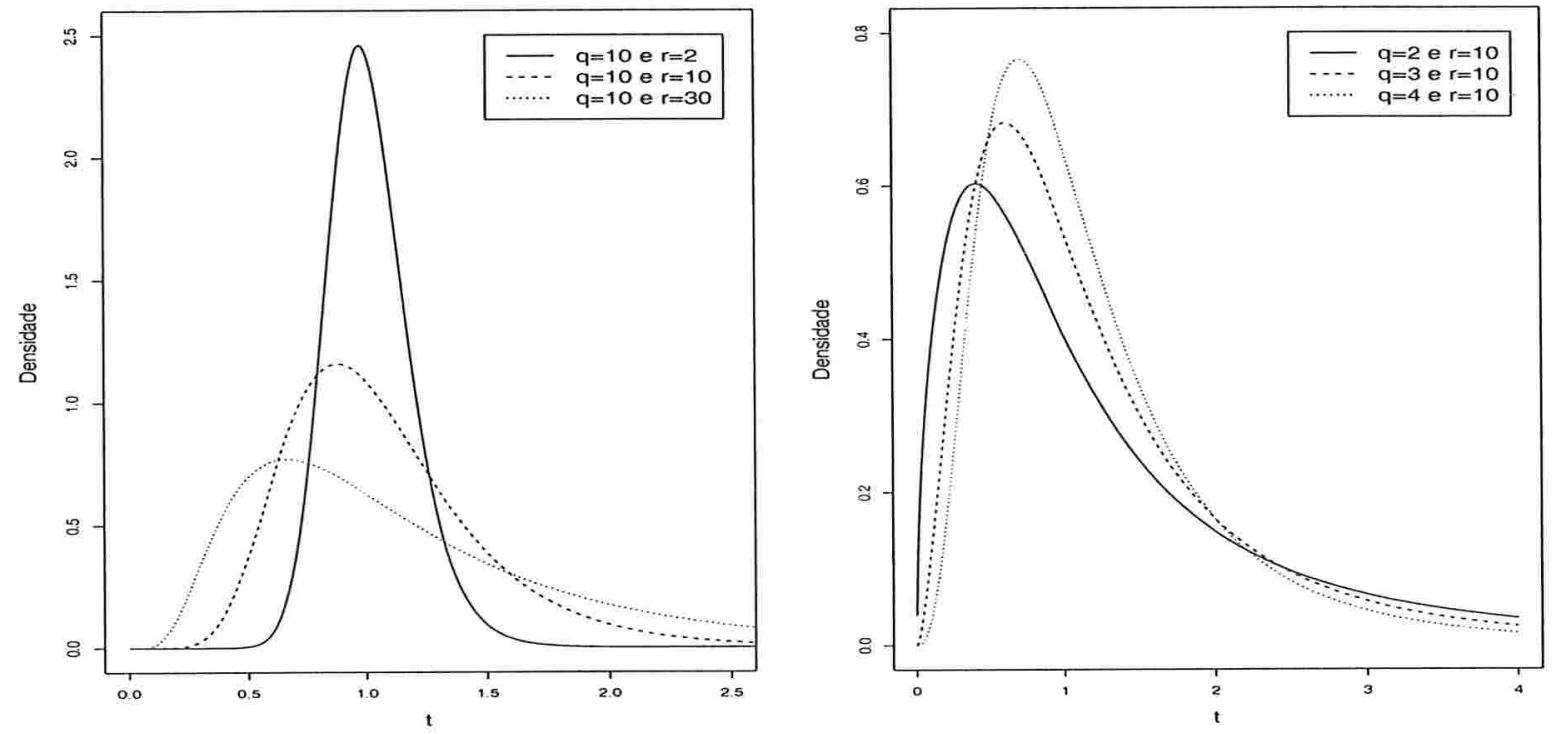

Figura 3.1: gráficos das funções densidade da distribuição Birnbaum-Saunders generalizada para $\alpha=0,5$ e $\beta=1,0$ obtidas a partir da distribuição Pearson VII com parâmetros $r$ e $q$.

A função de distribuição acumulada da distribuição BSG pode ser expressa da seguinte forma:

$$
F_{T}(t ; \alpha, \beta)=F\left(a_{t}(\alpha, \beta)\right)
$$

em que $F$ é a fda obtida a partir de (3.2) e $a_{t}(\alpha, \beta)$ é dada em (3.5), ou seja, a função de distribuição acumulada da distribuição BSG pode ser obtida em termos da função de distribuição acumulada da distribuição que a gera. Segue então que a função de sobrevivência 

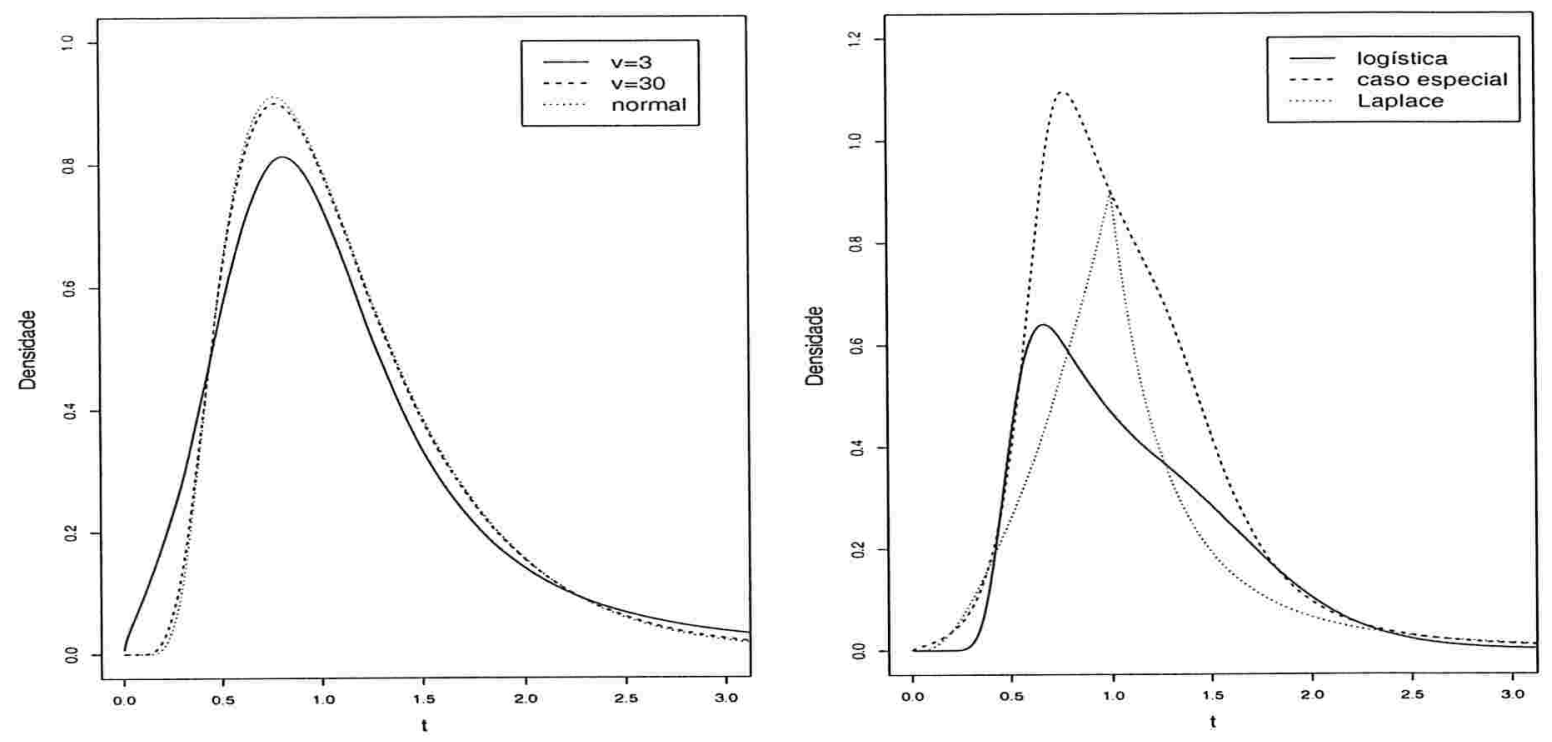

Figura 3.2: gráficos das funções densidade da distribuição Birnbaum-Saunders generalizada para $\alpha=0,5$ e $\beta=1,0$ obtidas a partir das distribuições $t$ de Student com 3 e 30 g.l., normal, logística, caso especial e Laplace.
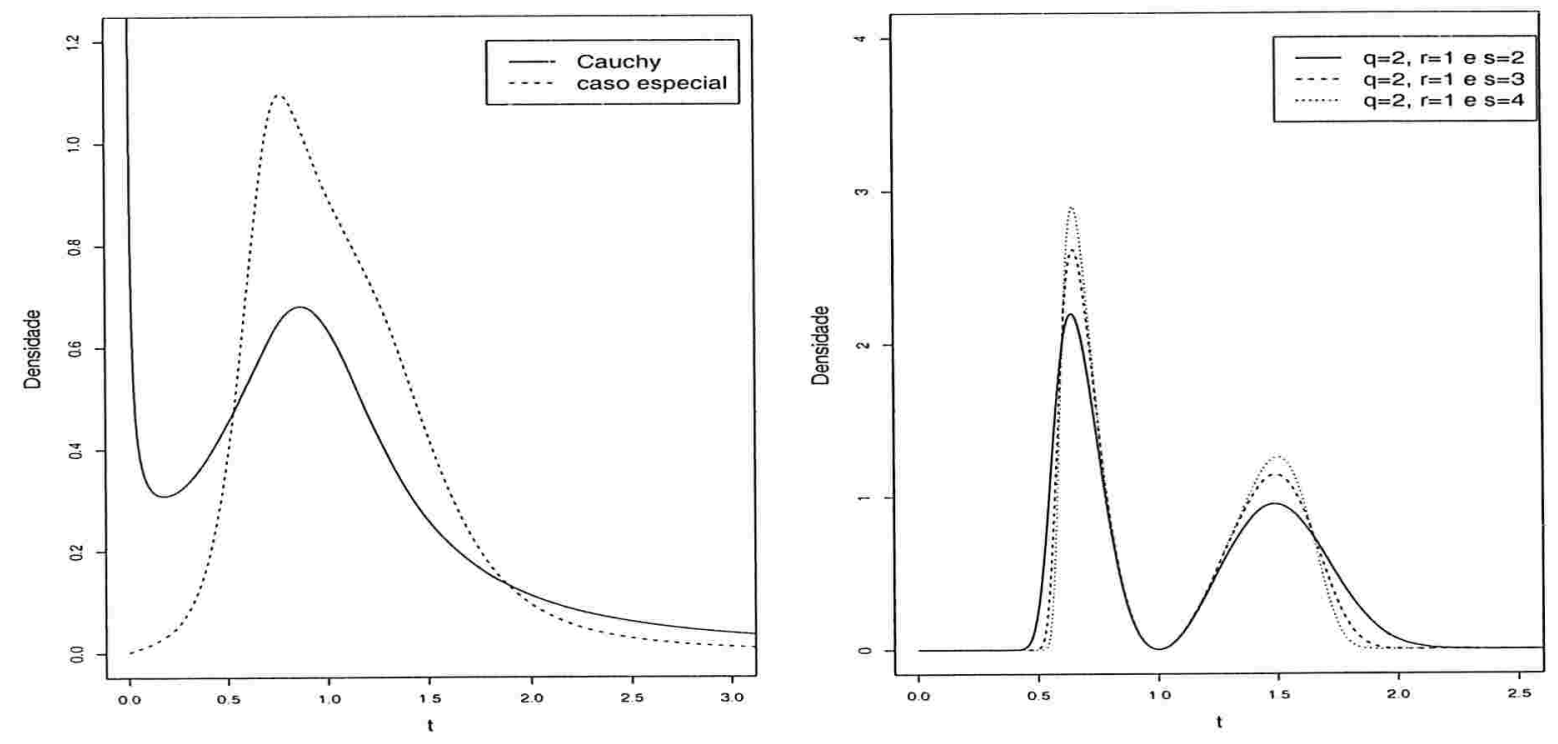

Figura 3.3: gráficos das funções densidade da distribuição Birnbaum-Saunders generalizada para $\alpha=0,5$ e $\beta=1,0$ obtidas a partir das distribuições Cauchy, caso especial e Kotz para os parâmetros $q, r$ e $s$ indicados. 

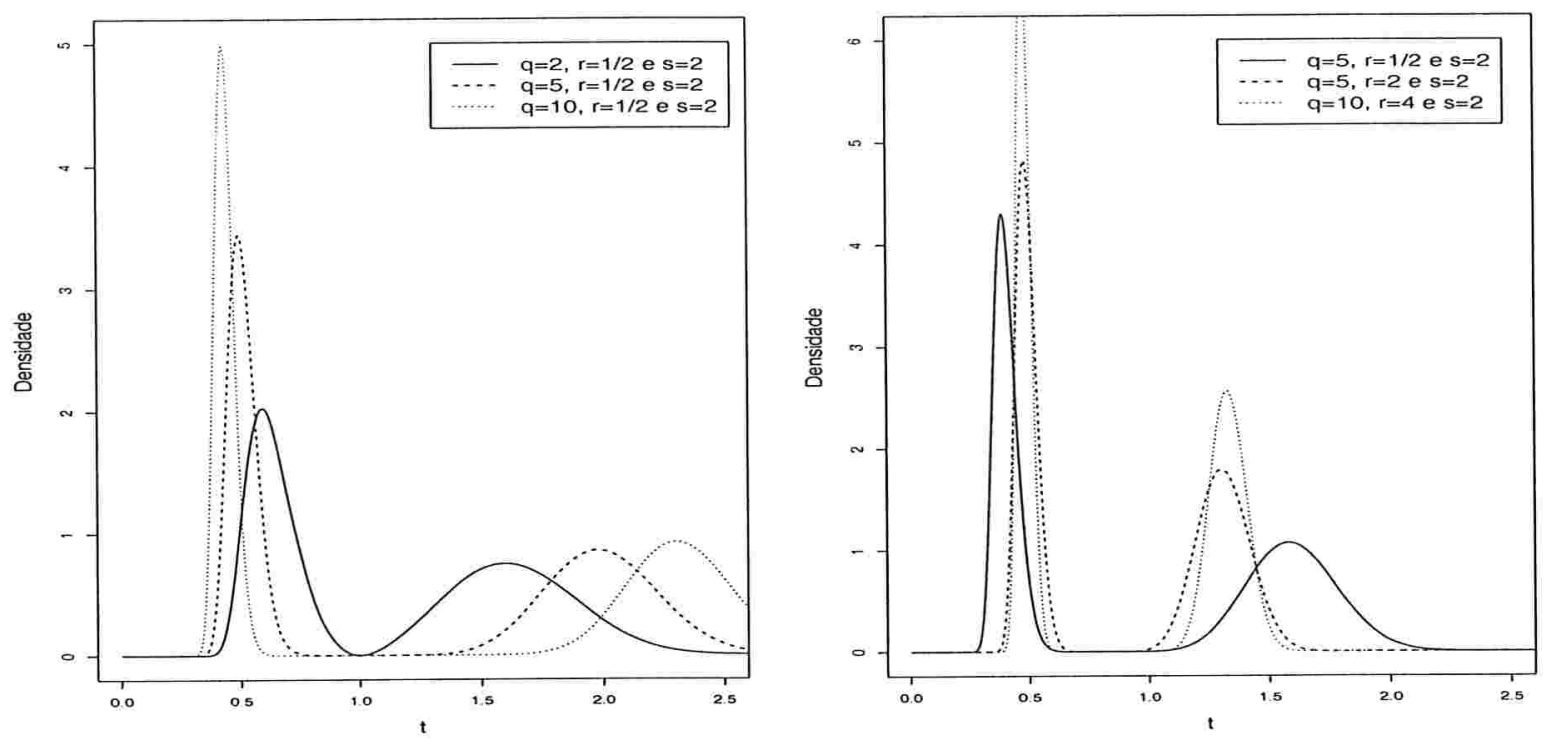

Figura 3.4: gráficos das funções densidade da distribuição Birnbaum-Saunders generalizada para $\alpha=0,5$ e $\beta=1,0$ obtidas a partir das distribuição Kotz com os parâmetros $q, r$ e $s$ indicados.

e a função de risco são dadas, respectivamente, por

$$
S_{T}(t ; \alpha, \beta)=1-F\left(a_{t}(\alpha, \beta)\right)
$$

$\mathrm{e}$

$$
h_{T}(t ; \alpha, \beta)=\frac{f_{T}(t ; \alpha, \beta)}{S_{T}(t ; \alpha \beta)}=\left(\frac{f\left(a_{t}(\alpha, \beta)\right)}{1-F\left(a_{t}(\alpha, \beta)\right.}\right) \frac{d}{d t} a_{t}(\alpha, \beta)
$$

Como ilustração, temos os gráficos da função de risco da distribuição Birnbaum-Saunders generalizada gerada pelas distribuições $t$ de Student, Cauchy e logística (Figuras 3.5 e 3.6). Como podemos observar, através das Figuras 3.5 e 1.2, a função de risco da distribuição BSG gerada pela distribuição $t$ de Student tem comportamento semelhante a da distribuição BS clássica, porém para graus de liberdade pequenos, o risco sobe rapidamente no início do período de observação, e cai rapidamente depois. No caso da distribuição BSG gerada pela distribuição Cauchy observamos que, para valores de $\alpha=0,50,0,75$ e 1,00 o risco é decrescente no início do período de observação, depois passa a ser crescente, atinge um valor máximo e depois decresce rapidamente; por outro lado, para valores de $\alpha=1,25,1,50$ e 2, 00, a função de risco é decrescente (ver Figura 3.6(a)). Para a distribuição BSG gerada pela distribuição logística 
temos que o risco é crescente no início do período de observação e depois decresce (Figura $3.6(b))$.
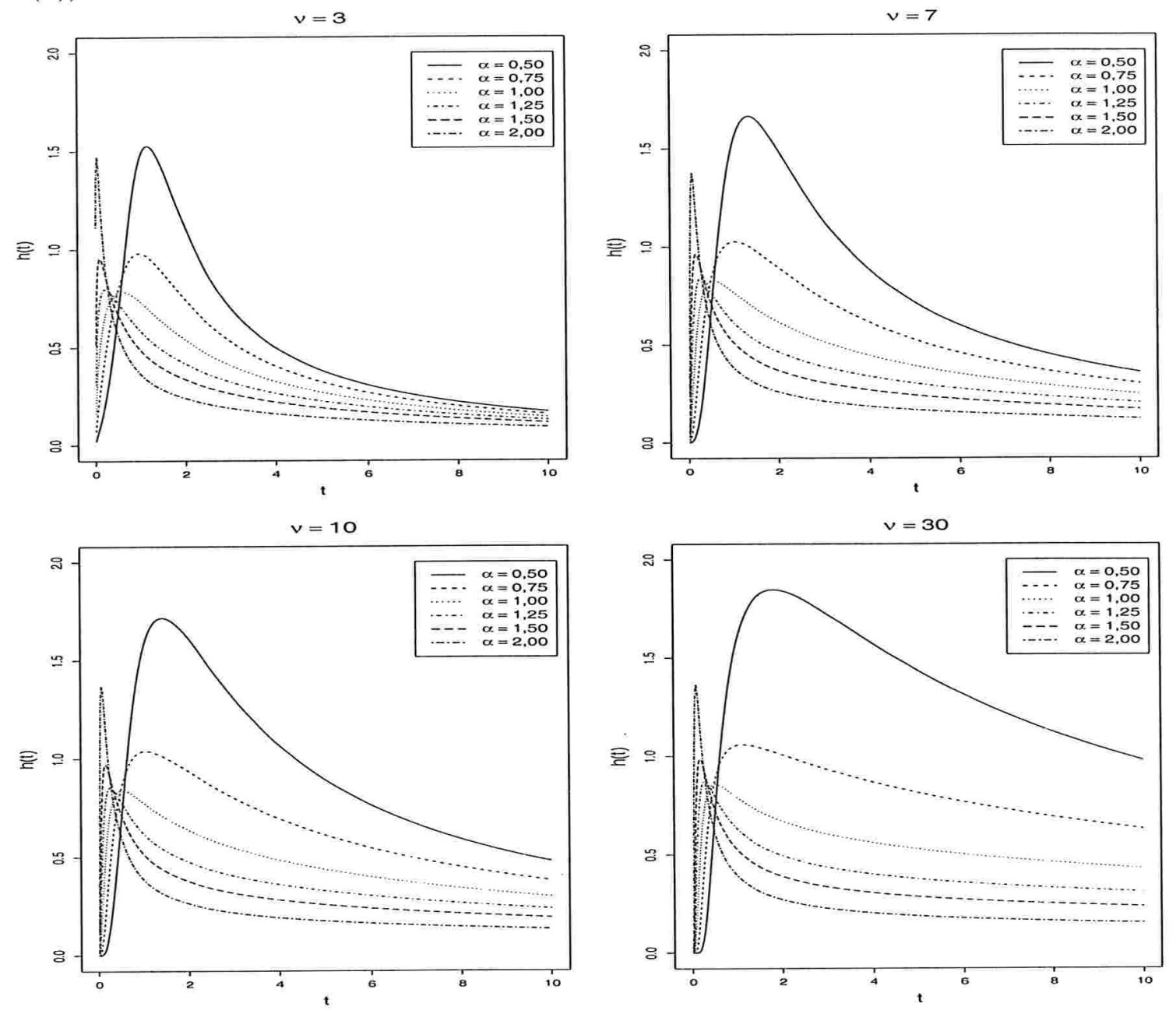

Figura 3.5: gráficos das funções de risco da distribuição Birnbaum-Saunders generalizada para $\beta=1,0$ e valores de $\alpha$ indicados, obtidas a partir da distribuição $t$ de Student com parâmetros $\nu=3, \nu=7, \nu=10$ e $\nu=30$.

É importante conhecer algumas propriedades da distribuição BSG para determinar seu comportamento sob diferentes escolhas de distribuições que a geram. Assim como no caso da distribuição Birnbaum-Saunders clássica, temos que se $T \sim B S G(\alpha, \beta ; g)$, então $c T$, com $c>0$, também segue uma distribuição BSG com parâmetros $\alpha$ e $c \beta$. Além disso, $T^{-1} \sim B S G\left(\alpha, \beta^{-1} ; g\right)$ indica que a distribuição BSG também pertence à família de variáveis aleatórias fechada sob reciprocidade (veja Saunders, 1974).

Uma propriedade importante é que a distribuição BSG possui momentos se, e somente se, a distribuição elíptica correspondente que a gera possui momentos. Assim, se $T \sim$ 

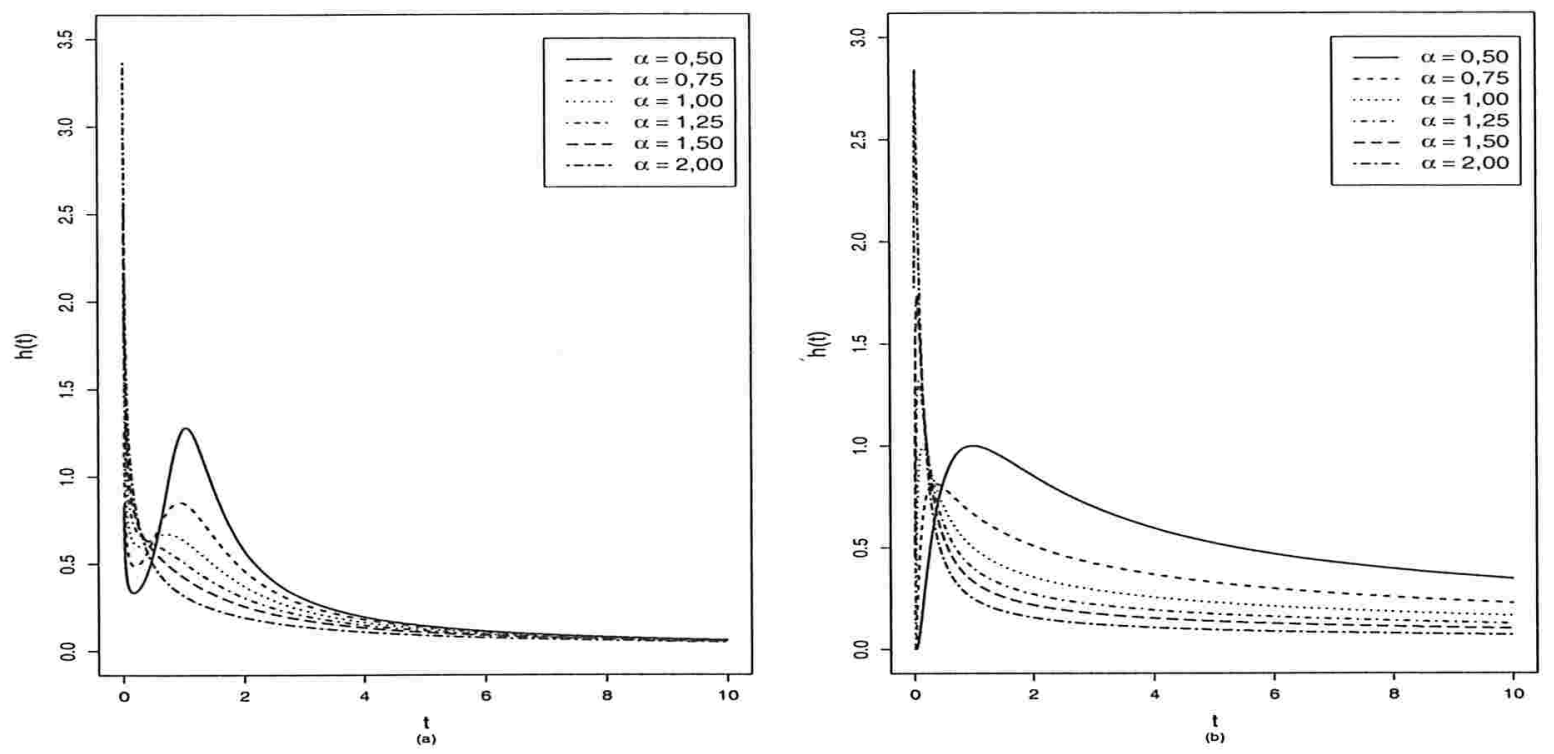

Figura 3.6: gráficos das funções de risco da distribuição Birnbaum-Saunders generalizada para $\beta=1,0$ e valores de $\alpha$ indicados, obtidas a partir das distribuições Cauchy (a) e logística (b).

$B S G(\alpha, \beta ; g)$ e esta possui momentos, então

$$
\mathbb{E}\left(\left[\frac{T}{\beta}\right]^{r}\right)=\sum_{k=0}^{r}\left(\begin{array}{c}
2 r \\
2 k
\end{array}\right) \sum_{s=0}^{k}\left(\begin{array}{c}
k \\
s
\end{array}\right) \frac{(2(r+s-k)) !}{(r+s-k) !} \frac{\phi^{(r+s-k)}(0)}{i^{2(r+s-k)}}\left(\frac{\alpha}{2}\right)^{2(r+s-k)},
$$

em que $\phi$ é dado em (3.1).

Os momentos com expoentes negativos da distribuição BSG podem ser obtidos através das propriedades de reciprocidade e proporcionalidade, ou seja, se $T \sim B S G(\alpha, \beta ; g)$, então $\beta T^{-1} \sim B S G(\alpha, 1 ; g)$. Segue, então, que as variáveis aleatórias $\beta T^{-1}$ e $\beta^{-1} T$ têm a mesma distribuição e, portanto,

$$
\mathbb{E}\left(T^{-n}\right)=\frac{\mathbb{E}\left(T^{n}\right)}{\beta^{2 n}}
$$

O teorema a seguir apresenta uma forma alternativa para encontrar os momentos da distribuição BSG.

Teorema 3.1 Seja $T \sim B S G(\alpha, \beta ; g)$ e suponha que todos os momentos de $T$ existam. Então, o n-ésimo momento de $T$ é

$$
\mathbb{E}\left(T^{n}\right)=\frac{\alpha^{2 n} \beta^{n}}{2} \mathbb{E}\left(Z^{2 n}\right)-\frac{1}{2}\left\{\sum_{k=1}^{n}\left(\begin{array}{c}
2 n \\
k
\end{array}\right) \frac{\mathbb{E}\left(T^{n-k}\right)}{\beta^{-k}(-1)^{k}}+\beta^{2 n} \sum_{k=n+1}^{2 n-1}\left(\begin{array}{c}
2 n \\
k
\end{array}\right) \frac{\mathbb{E}\left(T^{k-n}\right)}{\beta^{k}(-1)^{k}}\right\} .
$$

para $n=1,2, \ldots$, em que $Z \sim E C(0,1 ; g)$. 
Prova. De (3.3) segue que $U=\frac{1}{\alpha}\left(\sqrt{\frac{T}{\beta}}-\sqrt{\frac{\beta}{T}}\right)$ e usando o teorema binomial obtemos

$$
\begin{aligned}
U^{2 n} & =\frac{1}{\alpha^{2 n} \beta^{n}} \frac{(T-\beta)^{2 n}}{T^{n}} \\
& =\frac{1}{\alpha^{2 n} \beta^{n}} \sum_{k=0}^{2 n}\left(\begin{array}{c}
2 n \\
k
\end{array}\right) T^{n-k} \beta^{k}(-1)^{k} .
\end{aligned}
$$

Aplicando o valor esperado em (3.13) e usando (3.11) temos

$$
\begin{aligned}
\mathbb{E}\left(U^{2 n}\right) & =\frac{1}{\alpha^{2 n} \beta^{n}}\left\{\mathbb{E}\left(T^{n}\right)+\sum_{k=1}^{n}\left(\begin{array}{c}
2 n \\
k
\end{array}\right) \mathbb{E}\left(T^{n-k}\right)(-\beta)^{k}+\sum_{k=n+1}^{2 n-1}\left(\begin{array}{c}
2 n \\
k
\end{array}\right) \mathbb{E}\left(T^{n-k}\right)(-\beta)^{k}+\mathbb{E}\left(T^{-n}\right) \beta^{2 n}\right\} \\
& =\frac{1}{\alpha^{2 n} \beta^{n}}\left\{2 \mathbb{E}\left(T^{n}\right)+\sum_{k=1}^{n}\left(\begin{array}{c}
2 n \\
k
\end{array}\right) \mathbb{E}\left(T^{n-k}\right) \beta^{k}(-1)^{k}+\sum_{k=n+1}^{2 n-1}\left(\begin{array}{c}
2 n \\
k
\end{array}\right) \mathbb{E}\left(T^{k-n}\right) \beta^{2(n-k)} \beta^{k}(-1)^{k}\right\} \\
& =\frac{1}{\alpha^{2 n} \beta^{n}}\left\{2 \mathbb{E}\left(T^{n}\right)+\sum_{k=1}^{n}\left(\begin{array}{c}
2 n \\
k
\end{array}\right) \frac{\mathbb{E}\left(T^{n-k}\right)}{\beta^{-k}(-1)^{k}}+\beta^{2 n} \sum_{k=n+1}^{2 n-1}\left(\begin{array}{c}
2 n \\
k
\end{array}\right) \frac{\mathbb{E}\left(T^{k-n}\right)}{\beta^{k}(-1)^{k}}\right\} .
\end{aligned}
$$

Finalmente,

$$
\mathbb{E}\left(T^{n}\right)=\frac{\alpha^{2 n} \beta^{n}}{2} \mathbb{E}\left(U^{2 n}\right)-\frac{1}{2}\left\{\sum_{k=1}^{n}\left(\begin{array}{c}
2 n \\
k
\end{array}\right) \frac{\mathbb{E}\left(T^{n-k}\right)}{\beta^{-k}(-1)^{k}}+\beta^{2 n} \sum_{k=n+1}^{2 n-1}\left(\begin{array}{c}
2 n \\
k
\end{array}\right) \frac{\mathbb{E}\left(T^{k-n}\right)}{\beta^{k}(-1)^{k}}\right\} .
$$

Baseado em (3.11) e (3.12) obtemos recursivamente os quatro primeiros momentos da distribuição BSG, dados por
(a) $\mathbb{E}(T)=\frac{\beta}{2}\left(2+V_{1} \alpha^{2}\right)$,
(b) $\mathbb{E}\left(T^{2}\right)=\frac{\beta^{2}}{2}\left(2+4 V_{1} \alpha^{2}+V_{2} \alpha^{4}\right)$,
(c) $\mathbb{E}\left(T^{3}\right)=\frac{\beta^{3}}{2}\left(2+9 V_{1} \alpha^{2}+6 V_{2} \alpha^{4}+V_{3} \alpha^{6}\right)$ e
(d) $\mathbb{E}\left(T^{4}\right)=\frac{\beta^{4}}{2}\left(2+16 V_{1} \alpha^{2}+20 V_{2} \alpha^{4}+8 V_{3} \alpha^{6}+V_{4} \alpha^{8}\right)$,
em que $V_{n}=\mathbb{E}\left(U^{2 n}\right)$ e $U \sim E C(0,1 ; g)$.

Na Tabela 3.2 encontram-se expressões para $\mathbb{E}\left(U^{2 n}\right)=V_{n}$, com $n=1,2,3,4$, para as distribuições elípticas indicadas. Conseqüentemente, a partir da Tabela 3.2 podemos especificar (a), (b), (c) e (d).

A partir de (a), (b), (c) e (d) podemos obter os coeficientes de variação, assimetria e curtose dados, respectivamente, por 
Tabela 3.2: $\mathbb{E}\left(U^{2 n}\right)$ em que $U \sim E C(0,1 ; g)$, com $n=1,2,3,4$, para as distribuições elípticas indicadas.

\begin{tabular}{lcccc}
\hline Distribuição geradora & $\mathbb{E}\left(U^{2}\right)$ & $\mathbb{E}\left(U^{4}\right)$ & $\mathbb{E}\left(U^{6}\right)$ & $\mathbb{E}\left(U^{8}\right)$ \\
\hline Kotz & $\frac{1}{r^{\frac{1}{s}} \frac{\Gamma\left(\frac{2 q+1}{2 s}\right)}{\Gamma\left(\frac{2 q-1}{2 s}\right)}}$ & $\frac{1}{r^{\frac{2}{3}} \frac{\Gamma\left(\frac{2 q+3}{2 s}\right)}{\Gamma\left(\frac{2 q-1}{2 s}\right)}}$ & $\frac{1}{r^{\frac{3}{s}} \frac{\Gamma\left(\frac{2 q+5}{2 s}\right)}{\Gamma\left(\frac{2 q-1}{2 s}\right)}}$ & $\frac{1}{r^{\frac{4}{s}} \frac{\Gamma\left(\frac{2 q+7}{2 s}\right)}{\Gamma\left(\frac{2 q-1}{2 s}\right)}}$ \\
Exponencial Potência & $\frac{2^{\frac{1}{\eta}} \Gamma\left(\frac{3}{2 \eta}\right)}{\Gamma\left(\frac{1}{2 \eta}\right)}$ & $\frac{2^{\frac{2}{\eta}} \Gamma\left(\frac{5}{2 \eta}\right)}{\Gamma\left(\frac{1}{2 \eta}\right)}$ & $\frac{2^{\frac{3}{\eta}} \Gamma\left(\frac{7}{2 \eta}\right)}{\Gamma\left(\frac{1}{2 \eta}\right)}$ & $\frac{2^{\frac{4}{\eta}} \Gamma\left(\frac{9}{2 \eta}\right)}{\Gamma\left(\frac{1}{2 \eta}\right)}$ \\
Laplace & $2 !$ & $4 !$ & $6 !$ & $8 !$ \\
Normal & 1 & 3 & 15 & 105 \\
\hline Pearson VII & $\frac{r}{2 q-3}$ & $\frac{3 r^{2}}{(2 q-3)(2 q-5)}$ & $\frac{15 r^{3}}{(2 q-3)(2 q-5)(2 q-7)}$ & $\frac{105 r^{4}}{(2 q-3)(2 q-5)(2 q-7)(2 q-9)}$ \\
& $q>\frac{3}{2}$ & $q>\frac{5}{2}$ & $q>\frac{7}{2}$ & $q>\frac{9}{2}$ \\
$t$ de Student & $\frac{\nu}{\nu-2}$ & $\frac{3 \nu^{2}}{(\nu-2)(\nu-4)}$ & $\frac{15 \nu^{3}}{(\nu-2)(\nu-4)(\nu-6)}$ & $\frac{105 \nu^{4}}{(\nu-2)(\nu-4)(\nu-6)(\nu-8)}$ \\
& $\nu>2$ & $\nu>4$ & $\nu>6$ & $\nu>8$ \\
\hline Logística ${ }^{a}$ & $\approx 0,7957$ & $\approx 1,5097$ & $\approx 4,2777$ & $\approx 0,0095$ \\
\hline
\end{tabular}

${ }^{a}$ Os momentos da distribuição logística foram calculados usando métodos numéricos.

$$
\gamma(T)=\frac{\sigma}{\mu}, \quad \alpha_{3}(T)=\sqrt{\beta_{1}(T)}=\frac{\mu_{3}}{\left(\mu_{2}\right)^{\frac{3}{2}}}, \quad \text { e } \quad \alpha_{4}(T)=\beta_{2}(T)=\frac{\mu_{4}}{\left(\mu_{2}\right)^{2}}
$$

em que $\mu_{j}=\mathbb{E}\left[(T-\mathbb{E}[T])^{j}\right]$, com $j=2,3,4, \sigma=\sqrt{\operatorname{Var}(T)}=\sqrt{\mu_{2}}$, e $\mu=\mathbb{E}(T)$. O teorema a seguir nos fornece expressões para esses coeficientes.

Teorema 3.2 Seja $T \sim \operatorname{BSG}(\alpha, \beta ; g)$ e suponhamos que $\mathbb{E}\left(T^{n}\right)$ exista. Então, a variância e os coeficientes de variação, assimetria e curtose de $T$ são dados, respectivamente, por

$$
\begin{aligned}
\operatorname{Var}(T) & =\frac{\beta^{2}}{4}\left(2 \alpha^{4} V_{2}+4 \alpha^{2} V_{1}-\alpha^{4} V_{1}^{2}\right), \\
\gamma(T) & =\frac{\left(2 V_{2} \alpha^{4}+4 V_{1} \alpha^{2}-V_{1}^{2} \alpha^{4}\right)^{\frac{1}{2}}}{\left(2+V_{1} \alpha^{2}\right)}
\end{aligned}
$$




$$
\alpha_{3}(T)=\frac{2\left(6 V_{2} \alpha+2 V_{3} \alpha^{3}-6 V_{1}^{2} \alpha-3 V_{1} V_{2} \alpha^{3}+V_{1}^{3} \alpha^{3}\right)}{\left(2 V_{2} \alpha^{2}+4 V_{1}-V_{1}^{2} \alpha^{2}\right)^{\frac{3}{2}}}
$$

$e$

$$
\alpha_{4}(T)=\frac{\left(16 V_{2}+\left(32 V_{3}-48 V_{1} V_{2}+24 V_{1}^{3}\right) \alpha^{2}+\left(8 V_{4}-16 V_{1} V_{3}+12 V_{1}^{2} V_{2}-3 V_{1}^{4}\right) \alpha^{4}\right.}{\left(2 V_{2} \alpha^{2}+4 V_{1}-V_{1}^{2} \alpha^{2}\right)^{2}}
$$

Prova. Uma vez que

$$
\sigma^{2}=\frac{\beta^{2}}{4}\left(2 \alpha^{4} V_{2}+4 \alpha^{2} V_{1}-\alpha^{4} V_{1}^{2}\right) \quad \text { e } \quad \mu=\frac{\beta}{2}\left(2+V_{1} \alpha^{2}\right)
$$

obtemos diretamente o coeficiente de variação. Por outro lado, temos que

$$
\mu_{3}=\mathbb{E}\left(T^{3}\right)-3 \mathbb{E}(T) \mathbb{E}\left(T^{2}\right)+2(\mathbb{E}(T))^{3},
$$

então, depois de alguns cálculos algébricos obtemos

$$
\mu_{3}=-\frac{3}{2} V_{1}^{2} \alpha^{4} \beta^{3}+\frac{3}{2} V_{2} \alpha^{4} \beta^{3}+\frac{1}{4} V_{1}^{3} \alpha^{6} \beta^{3}-\frac{3}{4} V_{1} V_{2} \alpha^{6} \beta^{3}+\frac{1}{2} V_{3} \alpha^{6} \beta^{3} .
$$

Substituindo $\sigma$ e $\mu_{3}$ em $\alpha_{3}(T)$ dado em (3.14) obtemos o coeficiente de assimetria.

Analogamente, dado que

$$
\mu_{4}=\mathbb{E}\left(T^{4}\right)-4 \mathbb{E}\left(T^{3}\right) \mathbb{E}(T)+6 \mathbb{E}\left(T^{2}\right)(\mathbb{E}(T))^{2}-3(\mathbb{E}(T))^{4},
$$

após algumas manipulações algébricas, segue que

$$
\begin{aligned}
\mu_{4}= & V_{2} \alpha^{4} \beta^{4}+\frac{3}{2} V_{1}^{3} \alpha^{6} \beta^{4}-3 V_{1} V_{2} \alpha^{6} \beta^{4}+2 V_{3} \alpha^{6} \beta^{4}-\frac{3}{16} V_{1}^{4} \alpha^{8} \beta^{4}+\frac{3}{4} V_{1}^{2} V_{2} \alpha^{8} \beta^{4} \\
& -V_{1} V_{3} \alpha^{8} \beta^{4}+\frac{1}{2} V_{4} \alpha^{8} \beta^{4} .
\end{aligned}
$$

E, finalmente, substituindo $\sigma$ e $\mu_{4}$ em $\alpha_{4}(T)$ dado em (3.14) obtemos o coeficiente de curtose.

A partir da Tabela 3.2 podemos especificar a média, a variância, o coeficiente de variação, o coeficiente de assimetria e o coeficiente de curtose para a distribuição BSG especificada. Obtemos expressões explícitas para a distribuição BSG gerada pelas distribuições normal (distribuição BS clássica), Laplace, logística e $t$ de Student. Esses resultados são apresentados nos corolários a seguir.

Corolário 3.1 (Normal). Seja $T \sim \operatorname{BSG}(\alpha, \beta ; g) \operatorname{com} g(\cdot)$ sendo o núcleo da distribuição normal. Então, a média, a variância e os coeficientes de variação, assimetria e curtose de $T$ são dados, respectivamente, por

$$
\begin{gathered}
\mathbb{E}(T)=\beta\left(1+\frac{1}{2} \alpha^{2}\right), \quad \operatorname{Var}(T)=\beta^{2} \alpha^{2}\left(1+\frac{5}{4} \alpha^{2}\right), \gamma(T)=\frac{\alpha \sqrt{4+5 \alpha^{2}}}{2+\alpha^{2}} \\
\alpha_{3}(T)=\frac{4 \alpha\left(6+11 \alpha^{2}\right)}{\left(4+5 \alpha^{2}\right)^{\frac{3}{2}}} \text { e } \alpha_{4}(T)=3+\frac{6 \alpha^{2}\left(93 \alpha^{2}+40\right)}{\left(5 \alpha^{2}+4\right)^{2}}=\frac{3\left(211 \alpha^{4}+120 \alpha^{2}+16\right)}{\left(5 \alpha^{2}+4\right)^{2}}
\end{gathered}
$$


Corolário 3.2 (Laplace). Seja $T \sim \operatorname{BSG}(\alpha, \beta ; g)$ com $g(\cdot)$ sendo o núcleo da distribuição Laplace. Então, a média, a variância e os coeficientes de variação, assimetria e curtose de $T$ são dados, respectivamente, por

$$
\begin{gathered}
\mathbb{E}(T)=\beta\left(1+\alpha^{2}\right), \quad \operatorname{Var}(T)=\beta^{2} \alpha^{2}\left(2+11 \alpha^{2}\right), \quad \gamma(T)=\frac{\alpha \sqrt{2+11 \alpha^{2}}}{1+\alpha^{2}} \\
\alpha_{3}(T)=\frac{2 \alpha\left(15+163 \alpha^{2}\right)}{\left(2+11 \alpha^{2}\right)^{\frac{3}{2}}} \quad \text { e } \quad \alpha_{4}(T)=\frac{3\left(8+436 \alpha^{2}+6263 \alpha^{4}\right)}{\left(2+11 \alpha^{2}\right)^{2}}
\end{gathered}
$$

Corolário 3.3 (Logística). Seja $T \sim \operatorname{BSG}(\alpha, \beta ; g)$ com $g(\cdot)$ sendo o núcleo da distribuição logística. Então, a média, a variância e os coeficientes de variação, assimetria e curtose de $T$ são dados, respectivamente, por

$$
\begin{gathered}
\mathbb{E}(T) \approx \beta\left(1+0,3979 \alpha^{2}\right), \operatorname{Var}(T) \approx \beta^{2} \alpha^{2}\left(0,7957+0,5966 \alpha^{2}\right), \\
\gamma(T) \approx \frac{\alpha \sqrt{0,7957+0,5966 \alpha^{2}}}{\left(1+0,3979 \alpha^{2}\right)}, \quad \alpha_{3}(T) \approx \frac{\alpha\left(1,3148+1,3635 \alpha^{2}\right)}{\left(0,7957+0,5966 \alpha^{2}\right)^{\frac{3}{2}}} \quad e \\
\alpha_{4}(T) \approx \frac{1,5097+5,7073 \alpha^{2}-2,7573 \alpha^{4}}{0,3559\left(1,3338+\alpha^{2}\right)^{2}} .
\end{gathered}
$$

Corolário 3.4 ( $t$ de Student). Seja $T \sim \operatorname{BSG}(\alpha, \beta ; g) \operatorname{com} g(\cdot)$ sendo o núcleo da distribuição $t$ de Student. Então, a média, a variância e os coeficientes de variação, assimetria e curtose de $T$ são dados, respectivamente, por

$$
\begin{gathered}
\mathbb{E}(T)=\beta\left(1+\frac{\nu \alpha^{2}}{2(\nu-2)}\right) ; \nu>2, \\
\operatorname{Var}(T)=\beta^{2} \alpha^{2}\left(\frac{\nu}{\nu-2}+\frac{5}{4} \frac{\nu^{2}\left(\nu-\frac{8}{5}\right)}{(\nu-2)^{2}(\nu-4)} \alpha^{2}\right) ; \nu>4, \\
\gamma(T)=\frac{\alpha \sqrt{4 \frac{\nu}{(\nu-2)}+5 \frac{\nu^{2}\left(\nu-\frac{8}{5}\right)}{(\nu-2)^{2}(\nu-4)} \alpha^{2}}}{2+\frac{\nu}{(\nu-2)} \alpha^{2}} ; \nu>4, \\
\alpha_{3}(T)=\frac{4\left(\frac{(\nu-1) \nu^{2}}{(\nu-6)(\nu-2)^{2}}\right) \alpha\left(72 \frac{1}{(\nu-4)(\nu-2)}+6 \frac{\nu(\nu-8)}{(\nu-4)(\nu-2)}+11 \frac{\nu\left(\nu-\frac{18}{11}\right)}{(\nu-2)(\nu-4)} \alpha^{2}\right)}{\left(4 \frac{\nu}{(\nu-2)}+5 \frac{\nu^{2}\left(\nu-\frac{8}{5}\right)}{(\nu-2)^{2}(\nu-4)} \alpha^{2}\right)^{\frac{3}{2}}} ; \nu>8
\end{gathered}
$$

e 


$$
\alpha_{4}(T)=\frac{3\left(A \alpha^{4}+B \alpha^{2}+C+D\right)}{\left(4 \frac{\nu}{(\nu-2)}+5 \frac{\nu^{2}\left(\nu-\frac{8}{5}\right)}{(\nu-2)^{2}(\nu-4)} \alpha^{2}\right)^{2}} ; \nu>8
$$

em que

$$
\begin{aligned}
A & =211\left(\frac{\nu^{7}-\frac{894}{211} \nu^{6}}{(\nu-8)(\nu-6)(\nu-4)(\nu-2)^{4}}\right)+\frac{1288 \nu^{5}-640 \nu^{4}-6144 \nu^{2}}{(\nu-8)(\nu-6)(\nu-4)(\nu-2)^{4}}, \\
B & =120\left(\frac{\nu^{5}-\frac{14}{5} \nu^{4}}{(\nu-6)(\nu-4)(\nu-2)^{3}}\right)+\frac{256 \nu^{3}}{(\nu-6)(\nu-4)(\nu-2)^{3}}, \\
C & =16\left(\frac{\nu^{7}-20 \nu^{6}+144 \nu^{5}-464 \nu^{4}}{(\nu-8)(\nu-6)(\nu-4)(\nu-2)^{3}}\right)+\frac{1108 \nu^{3}}{(\nu-8)(\nu-6)(\nu-4)(\nu-2)^{4}} e \\
D & =\frac{-6144 \nu^{2}}{(\nu-8)(\nu-6)(\nu-4)(\nu-2)^{4}} .
\end{aligned}
$$

Podemos observar que se $\nu \rightarrow \infty$, então as expressões $A, B, C$ e $D$ dadas em (3.19), (3.20), (3.21) e (3.22) convergem para 211, 120, 16 e zero, respectivamente. E portanto, quando $\nu \rightarrow \infty, \mathbb{E}(T), \operatorname{Var}(T), \gamma(T), \alpha_{3}(T)$ e $\alpha_{4}(T)$ convergem para os valores dados no caso da distribuição normal, como era de se esperar. Muitos dos resultados apresentados nesta seção, também, estão descritos em Leiva et al. (2007).

\subsection{Métodos de estimação}

Nesta seção os resultados da estimação dos parâmetros da distribuição BSG serão apresentados. Abordamos os métodos de máxima verossimilhança e dos momentos modificados. Para o método de máxima verossimilhança obteremos as funções escore e a matriz de informação observada. No caso do método dos momentos modificados, obteremos a distribuição assintótica dos estimadores. Apresentaremos uma proposta de correção de viés através do método bootstrap. Finalmente, apresentaremos resultados de simulação com respeito ao processo de estimação.

\subsubsection{Método de máxima verossimilhança}

Sejam $T_{1}, T_{2}, \ldots, T_{n}$ variáveis aleatórias independentes e identicamente distribuídas, em que cada $T_{i}, i=1, \ldots, n$, segue uma distribuição BSG com parâmetro de forma $\alpha$ e parâmetro 
de escala $\beta$. Baseado em (3.4) segue que o logaritmo da função de verossimilhança é dado por

$$
\begin{aligned}
l(\alpha, \beta) & =\sum_{i=1}^{n} \log f_{T}\left(t_{i} ; \alpha, \beta\right) \\
& \propto-n \log \alpha-\frac{n}{2} \log \beta-\frac{3}{2} \sum_{i=1}^{n} \log t_{i}+\sum_{i=1}^{n} \log \left(t_{i}+\beta\right)+\sum_{i=1}^{n} \log g\left(a_{t_{i}}^{2}(\alpha, \beta)\right) .
\end{aligned}
$$

Para a obtenção dos estimadores de máxima verossimilhança é necessário obter as funções escore, ou seja, as primeiras derivadas do logaritmo da função de verossimilhança com relação aos parâmetros desconhecidos $\alpha$ e $\beta$. Portanto, assumimos que tais derivadas existem. Assim, as distribuições elípticas que não satisfazem essa condição não serão consideradas.

As funções escore para $\alpha$ e $\beta$ são dadas, respectivamente, por

$$
U_{\alpha}(\alpha, \beta)=-\frac{n}{\alpha}-\frac{2}{\alpha} \sum_{i=1}^{n} W_{g}\left(a_{t_{i}}^{2}(\alpha, \beta)\right) a_{t_{i}}^{2}(\alpha, \beta)
$$

$\mathrm{e}$

$$
U_{\beta}(\alpha, \beta)=-\frac{n}{2 \beta}+\sum_{i=1}^{n} \frac{1}{t_{i}+\beta}-\frac{1}{\alpha^{2}} \sum_{i=1}^{n} W_{g}\left(a_{t_{i}}^{2}(\alpha, \beta)\right)\left(\frac{t_{i}}{\beta^{2}}-\frac{1}{t_{i}}\right),
$$

em que $W_{g}(\cdot)=\frac{g^{\prime}(\cdot)}{g(\cdot)}$.

Em seguida obtemos a matriz de informação de Fisher observada, uma vez que não temos a matriz de informação de Fisher esperada. Para isto, é necessário o cálculo das segundas derivadas. Inicialmente, a partir da expressão (3.23), segue que a segunda derivada de $l(\alpha, \beta)$ com relação a $\alpha$ é dada por

$$
\ddot{L}_{\alpha, \alpha}=\frac{n}{\alpha^{2}}+\frac{6}{\alpha^{2}} \sum_{i=1}^{n} W_{g}\left(a_{t_{i}}^{2}(\alpha, \beta)\right) a_{t_{i}}^{2}(\alpha, \beta)+\frac{4}{\alpha^{2}} \sum_{i=1}^{n} W_{g}^{\prime}\left(a_{t_{i}}^{2}(\alpha, \beta)\right) a_{t_{i}}^{4}(\alpha, \beta) .
$$

A segunda derivada de $l(\alpha, \beta)$ com respeito a $\alpha$ e $\beta$ é dada por

$$
\begin{aligned}
\ddot{L}_{\alpha, \beta}=\ddot{L}_{\beta, \alpha} & =\frac{2}{\alpha^{3}} \sum_{i=1}^{n} W_{g}^{\prime}\left(a_{t_{i}}^{2}(\alpha, \beta)\right) a_{t_{i}}^{2}(\alpha, \beta)\left(\frac{t_{i}}{\beta^{2}}-\frac{1}{t_{i}}\right) \\
& +\frac{2}{\alpha^{3}} \sum_{i=1}^{n} W_{g}\left(a_{t_{i}}^{2}(\alpha, \beta)\right)\left(\frac{t_{i}}{\beta^{2}}-\frac{1}{t_{i}}\right)
\end{aligned}
$$

Finalmente, a partir de (3.24) obtemos a segunda derivada de $l(\alpha, \beta)$ com respeito a $\beta$ e 
esta é dada por

$$
\begin{aligned}
\ddot{L}_{\beta, \beta} & =\frac{n}{2 \beta^{2}}-\sum_{i=1}^{n}\left(\frac{1}{t_{i}+\beta}\right)^{2}+\frac{1}{\alpha^{4}} \sum_{i=1}^{n} W_{g}^{\prime}\left(a_{t_{i}}^{2}(\alpha, \beta)\right)\left(\frac{t_{i}}{\beta^{2}}-\frac{1}{t_{i}}\right)^{2} \\
& +\frac{2}{\alpha^{2} \beta^{3}} \sum_{i=1}^{n} W_{g}\left(a_{t_{i}}^{2}(\alpha, \beta)\right) t_{i} .
\end{aligned}
$$

Assim a matriz de informação de Fisher observada para $(\alpha, \beta)$ é dada por

$$
\ddot{\boldsymbol{L}}=-\left[\begin{array}{ll}
\ddot{L}_{\alpha, \alpha} & \ddot{L}_{\alpha, \beta} \\
\ddot{L}_{\beta, \alpha} & \ddot{L}_{\beta, \beta}
\end{array}\right] .
$$

Os estimadores de máxima verossimilhança de $\alpha$ e $\beta$ são soluções das equações $U_{\alpha}(\alpha, \beta)=$ 0 e $U_{\beta}(\alpha, \beta)=0$. Porém, neste caso, essas equações também não apresentam soluções analíticas, sendo necessária a utilização de método iterativo para a obtenção das raízes.

\subsubsection{Método dos momentos modificado}

No caso da distribuição Birnbaum-Saunders clássica, tem-se que os estimadores obtidos pelo método dos momentos convencional para $\alpha$ e $\beta$ nem sempre existem e, quando eles existem podem não ser únicos ( $\mathrm{Ng}$, Kundu e Balakrishnan, 2003). Diante disto, os autores acima propuseram o método dos momentos modificado. Tal método consiste em igualar $\mathbb{E}(T)$ e $\mathbb{E}\left(T^{-1}\right)$ aos seus respectivos momentos amostrais, para obter os estimadores de $\alpha$ e $\beta$. Seguimos essa proposta para o caso da distribuição Birnbaum-Saunders generalizada.

Sejam $\left\{T_{1}, T_{2}, \ldots, T_{n}\right\}$ uma amostra aleatória de tamanho $n$ de uma distribuição Birnbaum-Saunders generalizada com função densidade de probabilidade dada em (3.4). As médias aritmética e harmônica amostrais são definidas, respectivamente, por

$$
S=\frac{1}{n} \sum_{i=1}^{n} T_{i} \quad \text { e } \quad R=\left[\frac{1}{n} \sum_{i=1}^{n} T_{i}^{-1}\right]^{-1} .
$$

Neste caso, o método dos momentos modificado consiste na obtenção de estimadores para $\alpha$ e $\beta$ resolvendo-se as equações

$$
S=\mathbb{E}(T) \quad \text { e } \quad R^{-1}=\mathbb{E}\left(T^{-1}\right),
$$

em que $\mathbb{E}(T)$ e $\mathbb{E}\left(T^{-1}\right)$ são obtidos, respectivamente, a partir de (3.10) e (3.11). Desta forma os estimadores para $\alpha$ e $\beta$, obtidos pelo método dos momentos modificados são dados por 


$$
\tilde{\alpha}=\left\{\frac{1-(S / R)^{1 / 2}}{\phi^{(1)}(0)}\right\}^{1 / 2}
$$

$\mathrm{e}$

$$
\tilde{\beta}=(S R)^{1 / 2} \text {. }
$$

Segue da lei fraca dos grandes números que

$$
S \stackrel{p}{\rightarrow} \mathbb{E}(T) \quad \text { e } \quad R^{-1} \stackrel{p}{\rightarrow} \mathbb{E}\left(T^{-1}\right), \text { quando } n \rightarrow \infty,
$$

e pelo teorema central do limite

$$
\sqrt{n}\left(\begin{array}{c}
S-\mathbb{E}(T) \\
R^{-1}-\mathbb{E}\left(T^{-1}\right)
\end{array}\right) \stackrel{D}{\longrightarrow} N_{2}[0 ; \Sigma], \text { quando } n \rightarrow \infty
$$

em que

$$
\Sigma=\left(\begin{array}{ll}
\sigma_{11} & \sigma_{12} \\
\sigma_{21} & \sigma_{22}
\end{array}\right)
$$

$\operatorname{com} \sigma_{11}=\operatorname{Var}(T), \sigma_{22}=\operatorname{Var}\left(T^{-1}\right)$ e $\sigma_{12}=\sigma_{21}=\operatorname{Cov}\left(T, T^{-1}\right)=1-\left(1-\phi^{(1)}(0) \alpha^{2}\right)^{2}$. Portanto, a distribuição conjunta assintótica de

$$
\left(\begin{array}{c}
\tilde{\alpha} \\
\tilde{\beta}
\end{array}\right)=\left(\begin{array}{l}
f_{1}(x, y) \\
f_{2}(x, y)
\end{array}\right)
$$

$\operatorname{com} f_{1}(x, y)=\left(\frac{1-(x y)^{1 / 2}}{\phi^{(1)}(0)}\right)^{1 / 2}$ e $f_{2}(x, y)=\left(\frac{x}{y}\right)^{1 / 2}$ é dada por

$$
\sqrt{n}\left(\begin{array}{c}
\tilde{\alpha}-\alpha \\
\tilde{\beta}-\beta
\end{array}\right) \stackrel{D}{\longrightarrow} N_{2}[0 ; \tilde{\Sigma}]
$$

em que

$$
\tilde{\Sigma}=\left.\left(\begin{array}{ll}
\frac{\partial f_{1}(x, y)}{\partial x} & \frac{\partial f_{1}(x, y)}{\partial y} \\
\frac{\partial f_{2}(x, y)}{\partial x} & \frac{\partial f_{2}(x, y)}{\partial y}
\end{array}\right) \Sigma\left(\begin{array}{ll}
\frac{\partial f_{1}(x, y)}{\partial x} & \frac{\partial f_{2}(x, y)}{\partial x} \\
\frac{\partial f_{1}(x, y)}{\partial y} & \frac{\partial f_{2}(x, y)}{\partial y}
\end{array}\right)\right|_{x=\mathbb{E}(T), y=\mathbb{E}\left(T^{-1}\right)},
$$

com 


$$
\begin{aligned}
& \frac{\partial f_{1}(x, y)}{\partial x}=-\frac{1}{4 \phi^{(1)}(0)}\left\{\frac{1-(x y)^{1 / 2}}{\phi^{(1)}(0)}\right\}^{-1 / 2}\left(\frac{x}{y}\right)^{-1 / 2} \\
& \frac{\partial f_{1}(x, y)}{\partial y}=-\frac{1}{4 \phi^{(1)}(0)}\left\{\frac{1-(x y)^{1 / 2}}{\phi^{(1)}(0)}\right\}^{-1 / 2}\left(\frac{x}{y}\right)^{1 / 2} \\
& \frac{\partial f_{2}(x, y)}{\partial x}=\frac{1}{2 y}\left(\frac{x}{y}\right)^{-1 / 2} \mathrm{e} \\
& \frac{\partial f_{2}(x, y)}{\partial y}=-\frac{1}{2 y}\left(\frac{x}{y}\right)^{1 / 2}
\end{aligned}
$$

Após algumas manipulações algébricas obtemos

$$
\tilde{\Sigma}=\left(\begin{array}{cc}
\frac{\alpha^{2}\left(3 \phi^{(2)}(0) \alpha^{2}-\left(\phi^{(1)}(0)\right)^{2}\right)}{4\left(\phi^{(1)}(0)\right)^{2}} & 0 \\
0 & \frac{\beta^{2} \alpha^{2}\left(3 \alpha^{2} \phi^{(2)}(0)-2 \phi^{(1)}(0)\right)}{\left(1-\phi^{(1)}(0) \alpha^{2}\right)^{2}}
\end{array}\right)
$$

ou seja, $\tilde{\alpha}$ e $\tilde{\beta}$ são assintoticamente ortogonais.

É válido salientar que os resultados obtidos aqui se reduzem aos obtidos em $\mathrm{Ng}$, Kundu e Balakrishnan (2003) quando consideramos $g$ como sendo o núcleo da função densidade da distribuição normal.

\subsection{Avaliação numérica}

Para uma primeira análise do desempenho dos estimadores consideramos a distribuição Birnbaum-Saunders generalizada obtida pelas distribuições elípticas $t$ de Student, $\operatorname{com} \nu=5$ e $\nu=7$ e Pearson VII, com parâmetros $q=4, r=6$ e $q=10, r=20$, cujas densidades são dadas na Tabela 3.1. Tal análise foi feita através de simulações de Monte Carlo para diferentes tamanhos amostrais. Todo o procedimento de cálculo foi programado na linguagem de programação Ox (Doornik, 2001).

Em nosso experimento consideramos os tamanhos de amostra $n=10,20,40$ e 60 e o número de réplicas de Monte Carlo, NREP $=1000$. Consideramos como valores verdadeiros dos parâmetros $\alpha=0,1 ; 0,25 ; 0,5 ; 1,0$ e 2,0 e $\beta=1$. As estimativas de máxima verossimilhança dos parâmetros da distribuição são obtidas maximizando-se o logaritmo da função de verossimilhança através do método BFGS. Tomamos como valores iniciais para o processo de maximização $\alpha_{0}=\sqrt{\frac{1}{n} \sum_{i=1}^{n}\left(\frac{t_{i}}{\beta}+\frac{\beta}{t_{i}}-2\right)}$ para $\alpha$ e para $\beta$ a mediana amostral.

Para análise de resultados da estimação pontual foram calculados, para cada tamanho da amostra, a média, estimativas do viés relativo e erro quadrático médio das 1000 estimativas. 
Nas Tabelas B.1-B.8, apresentadas no Apêndice B, encontram-se as estatísticas obtidas das simulações para os estimadores dos parâmetros $\alpha$ e $\beta$. Podemos observar que $\alpha$ é muito mais viesado que $\beta$, em todas as situações consideradas e para ambos os métodos de estimação. Observamos também que, em módulo, as estimativas dos vieses relativos aumentam quando aumentamos o valor de $\alpha$, para $n$ fixo. Além disso, percebemos que as estimativas do viés relativo e do erro quadrático médio, de todas as estimativas dos parâmetros, diminuem à medida que aumentamos o tamanho de amostra, como era esperado. Compararando essas tabelas, observamos ainda que, em geral, o estimadores de máxima verossimilhança (EMV) apresentaram desempenho superior aos estimadores obtidos pelo método dos momentos modificado (EMM), com exceção do caso em que consideramos a distribuição Pearson VII ( $q=10$, $r=20)$ para tamanho de amostra $n=60$. É válido salientar que as estimativas de $\alpha$ e $\beta$ estão sendo influenciadas pelos parâmetros da distribuição que gera a distribuição BirnbaumSaunders generalizada. Por exemplo, no caso da distribuição $t$ de Student com $\nu=5$ (Tabela B.1), as estimativas dos vieses relativos para $\alpha$ são maiores do que quando consideramos $\nu=7$ (Tabela B.2). No caso da distribuição Pearson VII com $q=4$ e $r=6$ (Tabela B.3) temos que as estimativas dos vieses para $\alpha$ são menores do que quando consideramos $q=10$ e $r=20$ (Tabela B.4). Já com relação ao parâmetro $\beta$, ocorre o contrário quando consideramos a distribuição Pearson VII.

Uma vez que os estimadores do parâmetro $\alpha$ obtidos pelos métodos de máxima verossimilhanca e dos momentos modificado se mostraram muito viesados, utilizaremos o método bootstrap (que será considerado na próxima seção) na tentativa de corrigir o viés.

\subsection{Correção de viés por bootstrap}

O bootstrap é um método computacional de inferência estatística proposto, originalmente, por Efron (1979) que pode responder a muitas questões estatísticas reais sem a necessidade de exaustivos, complicados e muitas vezes inviáveis cálculos analíticos. A metodologia bootstrap permite a estimação de variâncias, intervalos de confiança, níveis descritivos e outras quantidades de interesse da inferência estatística através da reamostragem direta dos dados, que são tratados como se fossem a própria população (ver Ospina, 2004).

Seja $\boldsymbol{x}=\left(x_{1}, \ldots, x_{n}\right)$ uma amostra aleatória de uma variável aleatória $X$, cuja distribuição está determinada por sua função de distribuição acumulada $F$. Sejam $\theta=t(F)$, o parâmetro de interesse, e $\widehat{\theta}=S(x)$ um estimador de $\theta$. Podemos obter algumas propriedades distribucionais de $\widehat{\theta}$ utilizando o método bootstrap. O método consiste em obter, a partir da amostra original $x$, um grande número de amostras bootstrap $\boldsymbol{x}^{*}=\left(x_{1}^{*}, \ldots, x_{n}^{*}\right)$, calcular as respectivas réplicas 
bootstrap de $\widehat{\theta}, \widehat{\theta}^{*}=S\left(\boldsymbol{x}^{*}\right)$ e estimar, por exemplo, o viés e a variância de $\widehat{\theta}$ através do viés e da variância empíricos das réplicas $\widehat{\theta}^{*}$. Podemos, também, com base na distribuição empírica de $\widehat{\theta}^{*}$, estimar a função de distribuição de $\widehat{\theta}$. Existem duas formas de bootstrap que se difereciam quanto à obtenção da amostra, a saber: bootstrap não-paramétrico e bootstrap paramétrico. No primeiro caso, a amostra bootstrap $\boldsymbol{x}^{*}=\left(x_{1}^{*}, \ldots, x_{n}^{*}\right)$ é obtida de uma estimativa nãoparamétrica de $F$, a função de distribuição empírica de $X$, dada por

$$
\widehat{F}(t)=\frac{\#\left\{x_{i} \leq t\right\}}{n}, \quad i=1, \ldots, n,
$$

que atribui probabilidade $1 / n$ a cada valor de $x_{i}, i=1, \ldots, n$. Se $x^{*}$ é uma amostra aleatória de $\widehat{F}$ significa que $x^{*}$ é formada por $n$ observações extraídas com reposição da amostra original $\boldsymbol{x}$. No segundo caso, suponhamos que $X$ tem uma distribuição cuja forma funcional é conhecida, mas que depende de parâmetros desconhecidos, que podem ser estimados através da amostra $x$. Denotamos por $F_{\widehat{\theta}}$ a estimativa paramétrica de $F$. O bootstrap paramétrico caracteriza-se pela obtenção das amostras bootstrap de $F_{\widehat{\theta}}$ e não por amostragem com reposição de valores de $x$.

Denotamos o viés do estimador $\widehat{\theta}=S(\boldsymbol{x})$ por $B_{F}(\theta, \widehat{\theta})$, da forma

$$
B_{F}(\theta, \widehat{\theta})=\mathrm{E}_{F}[S(x)]-t(F) .
$$

Os estimadores de bootstrap do viés nas versões paramétrica e não-paramétrica são definidos substituindo a verdadeira distribuição $F$, que gerou a amostra original, pelas distribuições $F_{\widehat{\theta}}$ e $\widehat{F}$, respectivamente. Gerando $B$ amostras bootstrap $\left(x_{1}^{*}, \ldots, x_{B}^{*}\right)$ de forma independente e calculando as respectivas réplicas bootstrap $\left(\theta_{1}^{*}, \ldots, \theta_{B}^{*}\right)$, podemos aproximar as esperanças bootstrap $\mathrm{E}_{F_{\bar{\theta}}}[S(\boldsymbol{x})]$ e $\mathrm{E}_{\widehat{F}}[S(\boldsymbol{x})]$ pela média

$$
\widehat{\theta}^{*}(\cdot)=\frac{1}{B} \sum_{i=1}^{B} \theta_{i}^{*} .
$$

Então, os vieses estimados por bootstrap são dados por

$$
\begin{aligned}
\widehat{B}_{F_{\widehat{\theta}}}(\theta, \widehat{\theta}) & =\widehat{\theta}^{*}(\cdot)-S(\boldsymbol{x}), \\
\widehat{B}_{\widehat{F}}(\theta, \widehat{\theta}) & =\widehat{\theta}^{*}(\cdot)-S(\boldsymbol{x}) .
\end{aligned}
$$

Note que a diferença entre as estimativas dos vieses está na forma como são geradas as amostras bootstrap. A partir dessas estimativas são obtidos os estimadores corrigidos até segunda ordem por bootstrap (ver Efron e Tibishirani, 1993), dadas por

$$
\bar{\theta}_{1}=S(x)-\widehat{B}_{F_{\hat{\theta}}}(\theta, \widehat{\theta})=2 S(x)-\widehat{\theta}^{*}(\cdot),
$$

e

$$
\bar{\theta}_{2}=S(x)-\widehat{B}_{\widehat{F}}(\theta, \widehat{\theta})=2 S(x)-\widehat{\theta}^{*}(\cdot) .
$$




\subsubsection{Avaliação numérica}

Como os estimadores de máxima verossimilhança para $\alpha$ tiveram um desempenho superior aos estimadores obtidos pelo método dos momentos modificados, realizamos então a correção de viés, considerando apenas os estimadores de máxima verossimilhança.

O experimento foi realizado nas mesmas condições anteriores. Consideramos o método bootstrap não-paramétrico e o número de réplicas bootstrap $B=300$. Como podemos observar nas Tabelas B.1-B.4, houve, de fato, uma redução nos vieses. Os estimadores de máxima verossimilhança corrigidos por bootstrap (ECB) para o parâmetro $\alpha$ apresentaram desempenho melhor do que os estimadores de máxima verossimilhança, em todos os casos considerados. Quanto ao parâmetro $\beta$, observamos alguns casos em que os estimadores de máxima verossimilhança se apresentaram melhores, mas em geral, os estimadores corrigidos por bootstrap tiveram um bom desempenho. Nas Tabelas B.5-B.8 observamos uma diminuição no erro quadrático médio quando consideramos o estimador corrigido. 


\section{Capítulo 4}

\section{Modelos de Regressão}

\section{Log-Birnbaum-Saunders Generalizados}

Neste capítulo consideramos o caso em que o comportamento de uma variável com distribuição BSG possa ser explicado através de uma estrutura com covariáveis e parâmetros desconhecidos. Este é o contexto de um modelo de regressão Birnbaum-Saunders generalizado. Assim como é feito no caso da distribuição Birnbaum-Saunders clássica, consideramos o modelo de regressão log-Birnbaum-Saunders generalizado que será uma extensão do modelo de regressão log-linear, apresentado no Capítulo 2. Abordaremos ainda métodos de diagnóstico, como feito no Capítulo 2 para esta nova classe de modelos. Particularmente, daremos ênfase em investigar o modelo $t$ de Student log-Birnbaum-Saunders, para o qual mostraremos que as estimativas de máxima verossimilhança são robustas diante de observações extremas. Inicialmente, definiremos a distribuição log-Birnbaum-Saunders generalizada para, em seguida, desenvolver o modelo de regressão em que a variável resposta segue essa distribuição.

\subsection{Distribuição log-Birnbaum-Saunders generalizada}

Por analogia ao caso da distribuição senh-normal dada em (1.8), Díaz-García e DomínguezMolina (2006) obtiveram a distribuição seno hiperbólica esférica (senh-esférica), denotada por $S S(\alpha, \gamma, \sigma ; g)$, cuja densidade é dada por

$$
f_{Y}(y)=\frac{c}{\sigma}\left[\frac{2}{\alpha} \cosh \left(\frac{y-\gamma}{\sigma}\right)\right] g\left[\left(\frac{2}{\alpha} \operatorname{senh}\left(\frac{y-\gamma}{\sigma}\right)\right)^{2}\right],
$$

em que $y \in \mathbb{R}, \alpha>0, \gamma \in \mathbb{R}, \sigma>0, \gamma=\log \beta$ e $c$ e $g$ são dadas em (3.2).

Segue que, se $Y=\log (T)$, em que $T \sim B S G(\alpha, \beta ; g)$, então $Y \sim S S(\alpha, \gamma, 2 ; g)$. 
No caso particular em que $g$ é o núcleo de uma distribuição normal, ou seja, $T$ segue uma distribuição Birnbaum-Saunders clássica, temos que $g(u)=e^{-u / 2}$ e $c=\frac{1}{\sqrt{2 \pi}}$, então $f_{Y}(y)$ fica dada por

$$
f_{Y}(y)=\frac{1}{\alpha \sqrt{2 \pi}} \cosh \left(\frac{y-\gamma}{2}\right) \exp \left[-\frac{2}{\alpha^{2}} \operatorname{senh}^{2}\left(\frac{y-\gamma}{2}\right)\right],
$$

ou seja, $Y \sim S N(\alpha, \gamma, 2)$ (ver Rieck e Nedelman, 1991).

A função de distribuição acumulada e a função de sobrevivência de $Y$ são dadas, respectivamente, por

$$
F_{Y}(y)=F\left[\frac{2}{\alpha} \operatorname{senh}\left(\frac{y-\gamma}{\sigma}\right)\right]
$$

e

$$
S_{Y}(y)=F\left[-\frac{2}{\alpha} \operatorname{senh}\left(\frac{y-\gamma}{\sigma}\right)\right]
$$

em que F é a fda da distribuição elíptica que gera a distribuição BSG.

Na Figura 4.1, gráficos das funções densidade senh-esférica são mostrados para diferentes escolhas de $\alpha$ e $\nu$, sendo $\gamma=\log (\beta)=0$ e $\sigma=2$. Baseando-se nesta figura, é possível ver que a distribuição $t$ de Student log-BS (distribuição BSG gerada pela distribuição $t$ de Student) é muito flexível para modelar a curtose. A Figura 1 também nos permite estabelecer uma comparação entre as distribuições $t$ de Student $\log$-BS, $\log$-BS, normal e $t$ de Student. Os parâmetros $\alpha$ e $\gamma$ são os parâmetros de forma e posição, respectivamente, enquanto que o parâmetro $\alpha$ está associado à modalidade da distribuição (para $\alpha>2$ a distribuição torna-se bimodal) e o parâmetro $\nu$ da distribuição $t$ de Student está associado à curtose e também à dispersão.

\subsection{O modelo de regressão log-Birnbaum-Saunders ge- neralizado}

Estendendo o modelo (2.1), consideremos o modelo de regressão log-BSG definido por

$$
y_{i}=\mathbf{x}_{i}^{\top} \boldsymbol{\beta}+\varepsilon_{i}, \quad i=1, \ldots, n,
$$

em que $y_{i}$ é o logaritmo do tempo de sobrevivência ou do tempo de censura para a $i$-ésima unidade experimental, $\boldsymbol{\beta}=\left(\beta_{1}, \beta_{2}, \ldots, \beta_{p}\right)^{\top}$ é o vetor de parâmetros desconhecidos a ser estimado, $\mathbf{x}_{i}=\left(x_{i 1}, x_{i 2}, \ldots, x_{i p},\right)^{\top}$ são observações de $p$ variáveis explicativas e $\varepsilon_{i}$ são variáveis 

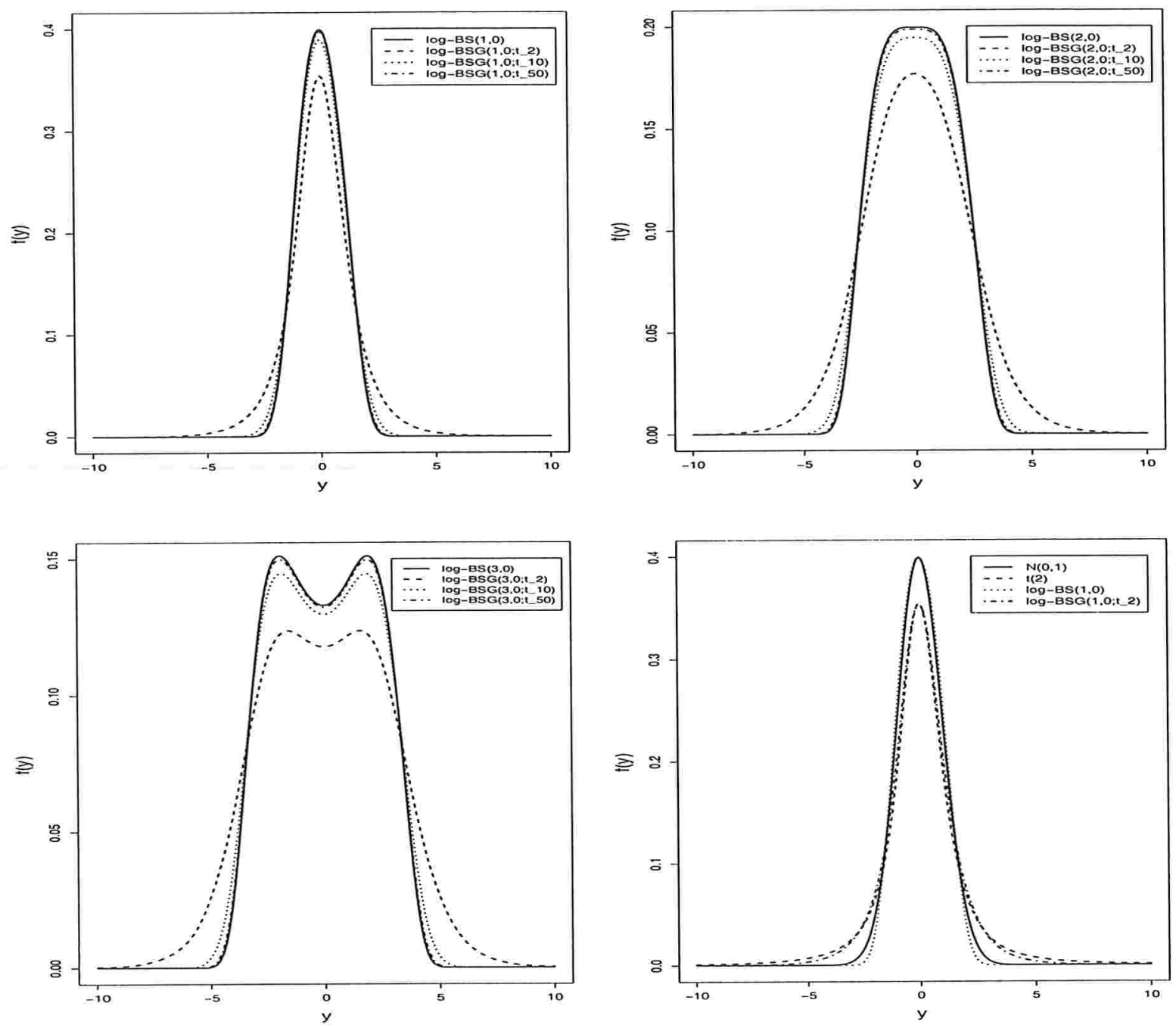

Figura 4.1: gráficos das funções densidade das distribuições log-BS, $t$ de Student log-BS, normal e $t$ de Student com parâmetros indicados. 
aleatórias independentes e identicamente distribuídas tais que $\varepsilon_{i} \sim \log -B S G(\alpha, 0 ; g)$. Mais uma vez, supomos censura não informativa e que os tempos de sobrevivência e censura são independentes. Denotaremos por $D$ e $C$ o conjunto de indivíduos para o qual $y_{i}$ é o logaritmo do tempo de sobrevivência ou o logaritmo do tempo censurado, respectivamente.

O logaritmo da função de verossimilhança total do modelo (4.5) para $\boldsymbol{\theta}=\left(\alpha, \boldsymbol{\beta}^{\top}\right)^{\top}$ fica dado por

$$
l(\boldsymbol{\theta}) \propto \sum_{i \in D}\left\{\log \left(\frac{c}{2}\right)+\log \left(\xi_{i 1}\right)+\log \left[g\left(\xi_{i 2}^{2}\right)\right]\right\}+\sum_{i \in C} \log \left[1-F\left(\xi_{i 2}\right)\right],
$$

em que $c$ e $g$ são dados em $(3.2), F(\cdot)$ é a fda da distribuição elíptica que gera a distribuição BSG, $\xi_{i 1}$ e $\xi_{i 2}$ são dados em (2.2).

Então, temos as funções escore

$$
U_{\alpha}(\boldsymbol{\theta})=\frac{1}{\alpha}\left[\sum_{i \in D}\left\{w\left(\xi_{i 2}^{2}\right) \xi_{i 2}^{2}-1\right\}+\sum_{i \in C} \xi_{i 2} h\left(\xi_{i 2}\right)\right]
$$

e

$$
U_{\beta_{j}}(\theta)=\sum_{i \in D}\left[\frac{x_{i j}}{\alpha^{2}} \operatorname{senh}\left(y_{i}-\mu_{i}\right) w\left(\xi_{i 2}^{2}\right)-\frac{x_{i j}}{2} \tanh \left(\frac{y_{i}-\mu_{i}}{2}\right)\right]+\frac{1}{2} \sum_{i \in C} x_{i j} \xi_{i 1} h\left(\xi_{i 2}\right),
$$

em que $w\left(\xi_{i 2}^{2}\right)=-2 W_{g}\left(\xi_{i 2}^{2}\right)$, com $W_{g}(\cdot)$ definido em (3.24) e $h(\cdot)=\frac{f\left(\xi_{i 2}\right)}{1-F\left(\xi_{i 2}\right)}$, com $f(\cdot)$ e $F(\cdot)$ denotando a fdp e fda da distribuição elíptica que gera a distribuição BSG. Expressões de $W_{g}(u)$ e $W_{g}^{\prime}(u)$ para algumas distribuições elípticas são dadas na Tabela 4.1. Note que a quantidade $w\left(\xi_{i 2}^{2}\right)$ que aparece nas funções escore (4.7) e (4.8) para as observações não censuradas pode ser interpretada como um tipo de peso no modelo log-BSG, uma vez que é inversamente proporcional a $\xi_{i 2}^{2}$ (com exceção da distribuição normal que é uma constante e da distribuição logística que é diretamente proporcional), observações com valores grandes para $\xi_{i 2}^{2}$ devem receber pesos menores no processo de estimação. A Figura 4.2 descreve o comportamento de $w\left(\xi_{i 2}^{2}\right)$ contra $\xi_{i 2}^{2}$ para alguns valores de graus de liberdade da distribuição $t$ de Student. Notamos que o peso decresce quando os graus de liberdade tornam-se menores. Estes resultados indicam aspecto de robustez dos estimadores de máxima verossimilhança $\widehat{\alpha} \mathrm{e}$ $\widehat{\boldsymbol{\beta}}$ contra observações não censuradas extremas, no que se diz respeito à quantidade $\xi_{i 2}$. Esses aspectos de robustez serão estendidos na Seção 4.3 para os resíduos componente do desvio e tipo martingale, usualmente aplicados em modelos de regressão de sobrevivência.

A matriz de informação de Fisher observada é dada por $-\ddot{\mathrm{L}}_{\theta \theta}$, em que

$$
\ddot{\mathrm{L}}_{\theta \theta}=\left[\begin{array}{cc}
\ddot{\mathrm{L}}_{\alpha \alpha} & \ddot{\mathrm{L}}_{\alpha \beta} \\
\ddot{\mathrm{L}}_{\beta \alpha} & \ddot{\mathrm{L}}_{\beta \beta}
\end{array}\right]=\left[\begin{array}{cc}
\operatorname{tr}(G) & \boldsymbol{K}^{\top} \boldsymbol{X} \\
\boldsymbol{X}^{\top} \boldsymbol{K} & \boldsymbol{X}^{\top} \boldsymbol{V} \boldsymbol{X}
\end{array}\right]
$$


Tabela 4.1: expressões para $W_{g}(u)$ e $W_{g}^{\prime}(u)$ para algumas distribuições elípticas

\begin{tabular}{lcc}
\hline Distribuição & $W_{g}(u)$ & $W_{g}^{\prime}(u)$ \\
\hline Normal & $-\frac{1}{2}$ & 0 \\
Pearson VII & $-\frac{q}{r\left(1+\frac{u}{r}\right)}$ & $\frac{q}{r^{2}\left(1+\frac{u}{r}\right)^{2}}$ \\
t-Student & $-\frac{(v+1)}{2(v+u)}$ & $\frac{(v+1)}{2(v+u)^{2}}$ \\
Cauchy & $-\frac{1}{(1+u)}$ & $\frac{(1)}{(1+u)^{2}}$ \\
Caso Especial & $-\frac{2 u}{1+u^{2}}$ & $-\frac{2\left(1-u^{2}\right)}{\left(1+u^{2}\right)^{2}}$ \\
Kotz & $\frac{\left[(q-1)-r s u^{s}\right]}{u^{2}}$ & $(1-s) r s u^{s-2}-\frac{(q-1)}{u^{2}}$ \\
Logística & $-\tanh ^{2}(u)$ & $-\operatorname{sech}^{2}(u)$ \\
\hline
\end{tabular}

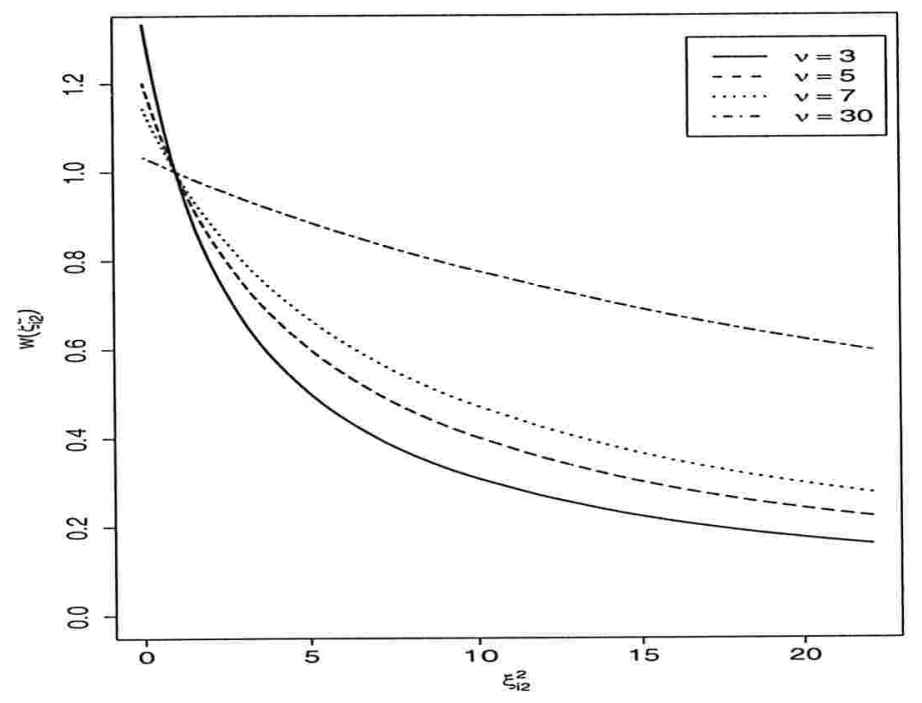

Figura 4.2: comportamento de $w\left(\xi_{i 2}^{2}\right)$ contra $\xi_{i 2}^{2}$ para alguns graus de liberdade da distribuição $t$ de Student.

$\operatorname{com} \boldsymbol{G}=\operatorname{diag}\left\{g_{1}(\boldsymbol{\theta}), \ldots, g_{n}(\boldsymbol{\theta})\right\} \mathrm{e}$

$$
g_{i}(\boldsymbol{\theta})=\left\{\begin{array}{l}
\frac{1}{\alpha^{2}}-\frac{3 \xi_{i 2}^{2}}{\alpha^{2}} w\left(\xi_{i 2}^{2}\right)-\frac{2}{\alpha^{2}} \xi_{i 2}^{4} w^{\prime}\left(\xi_{i 2}^{2}\right), \text { se } i \in D \\
-\frac{2}{\alpha^{2}} \xi_{i 2} h\left(\xi_{i 2}\right)-\frac{1}{\alpha^{2}} \xi_{i 2}^{2} h^{\prime}\left(\xi_{i 2}\right), \text { se } i \in C ;
\end{array}\right.
$$


$\boldsymbol{K}=\left(k_{1}(\boldsymbol{\theta}), \ldots, k_{n}(\boldsymbol{\theta})\right)^{\top}$, sendo que

$$
k_{i}(\boldsymbol{\theta})=\left\{\begin{array}{l}
-\frac{2}{\alpha^{3}} \operatorname{senh}\left(y_{i}-\mu_{i}\right)\left\{w\left(\xi_{i 2}^{2}\right)+\xi_{i 2}^{2} w^{\prime}\left(\xi_{i 2}^{2}\right)\right\}, \text { se } i \in D \\
-\frac{1}{2 \alpha} \xi_{i 1} h\left(\xi_{i 2}\right)-\frac{1}{\alpha^{3}} \operatorname{senh}\left(y_{i}-\mu_{i}\right) h^{\prime}\left(\xi_{i 2}\right), \text { se } i \in C
\end{array}\right.
$$

e $\boldsymbol{V}=\operatorname{diag}\left\{v_{1}(\boldsymbol{\theta}), \ldots, v_{n}(\boldsymbol{\theta})\right\}, \mathrm{com}$

$$
v_{i}(\boldsymbol{\theta})=\left\{\begin{array}{l}
\frac{1}{4} \operatorname{sech}^{2}\left(\frac{y_{i}-\mu_{i}}{2}\right)-\frac{1}{\alpha^{2}} \cosh \left(y_{i}-\mu_{i}\right) w\left(\xi_{i 2}^{2}\right)-\frac{2}{\alpha^{4}} \operatorname{senh}^{2}\left(y_{i}-\mu_{i}\right) w^{\prime}\left(\xi_{i 2}^{2}\right), \text { se } i \in D \\
-\frac{1}{4} \xi_{i 2} h\left(\xi_{i 2}\right)-\frac{1}{4} \xi_{i 1}^{2} h^{\prime}\left(\xi_{i 2}\right), \text { se } i \in C
\end{array}\right.
$$

em que $w^{\prime}(x)=d w(x) / d x$ e $h^{\prime}(x)=d h(x) / d x$.

Os estimadores de máxima verossimilhança dos coeficientes de regressão e do parâmetro de forma são soluções das equações $U_{\beta_{j}}(\theta)=0(j=1, \ldots, p)$ e $U_{\alpha}(\boldsymbol{\theta})=0$. Porém, como no modelo log-BS do Capítulo 2, as equações não apresentam soluções analíticas, sendo necessária, a utilização de métodos iterativos para a obtenção das raízes. Como valores iniciais no processo de estimação, pode-se usar as estimativas obtidas no modelo log-BS.

\subsection{Resíduos}

O resíduo componente do desvio, como vimos no Capítulo 2, toma a seguinte forma:

$$
\mathrm{r}_{D C_{i}}= \begin{cases}\operatorname{sinal}\left(\widehat{\xi}_{i 2}\right) \sqrt{2}\left[\log [g(0)]-\log \left[\cosh \left(\frac{y_{i}-\hat{\mu}_{i}}{2}\right)\right]-\log \left[g\left(\widehat{\xi}_{i 2}^{2}\right)\right]\right]^{\frac{1}{2}}, & \text { se } i \in D ; \\ \operatorname{sinal}\left(\widehat{\xi}_{i 2}\right)\left[-2 \log \left(1-F\left[\hat{\xi}_{i 2}\right]\right)\right]^{\frac{1}{2}}, & \text { se } i \in C,\end{cases}
$$

em que $g(\cdot)$ é o núcleo da fdp da distribuição elíptica que gera a distribuição BSG e $1-F(\cdot)$ é a função de sobrevivência do modelo log-BSG. Analogamente, o resíduo tipo martingale fica dado por

$$
\mathrm{r}_{M D_{i}}=\operatorname{sinal}\left(\mathrm{r}_{M_{i}}\right)\left\{-2\left[\mathrm{r}_{M_{i}}+\delta_{i} \log \left(\delta_{i}-\mathrm{r}_{M_{i}}\right)\right]\right\}^{\frac{1}{2}},
$$

em que $\mathrm{r}_{M_{i}}=\delta_{i}+\log \left\{1-F\left(\xi_{i 2}\right)\right\}$ com $\delta_{i}=0$ indicando se a observação é censurada, $\delta_{i}=1$ indicando se a observação é não censurada, $i=1, \ldots, n$.

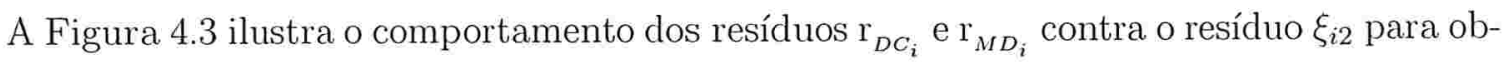
servações não censuradas quando consideramos o modelo $t$ de Student log-BS (modelo log-BSG 
cujo erro tem distribuição BSG gerada pela distribuição $t$ de Student) para alguns valores de $\nu$ e $\alpha=1$. Notamos em ambos os gráficos que observações não censuradas com valores grandes de $\mathrm{r}_{D C_{i}}$ e $\mathrm{r}_{M D_{i}}$ devem receber pesos menores. Portanto, o aspecto de robustez apresentado na seção anterior é também estendido para os resíduos $\mathrm{r}_{D C_{i}}$ e $\mathrm{r}_{M D_{i}}$.
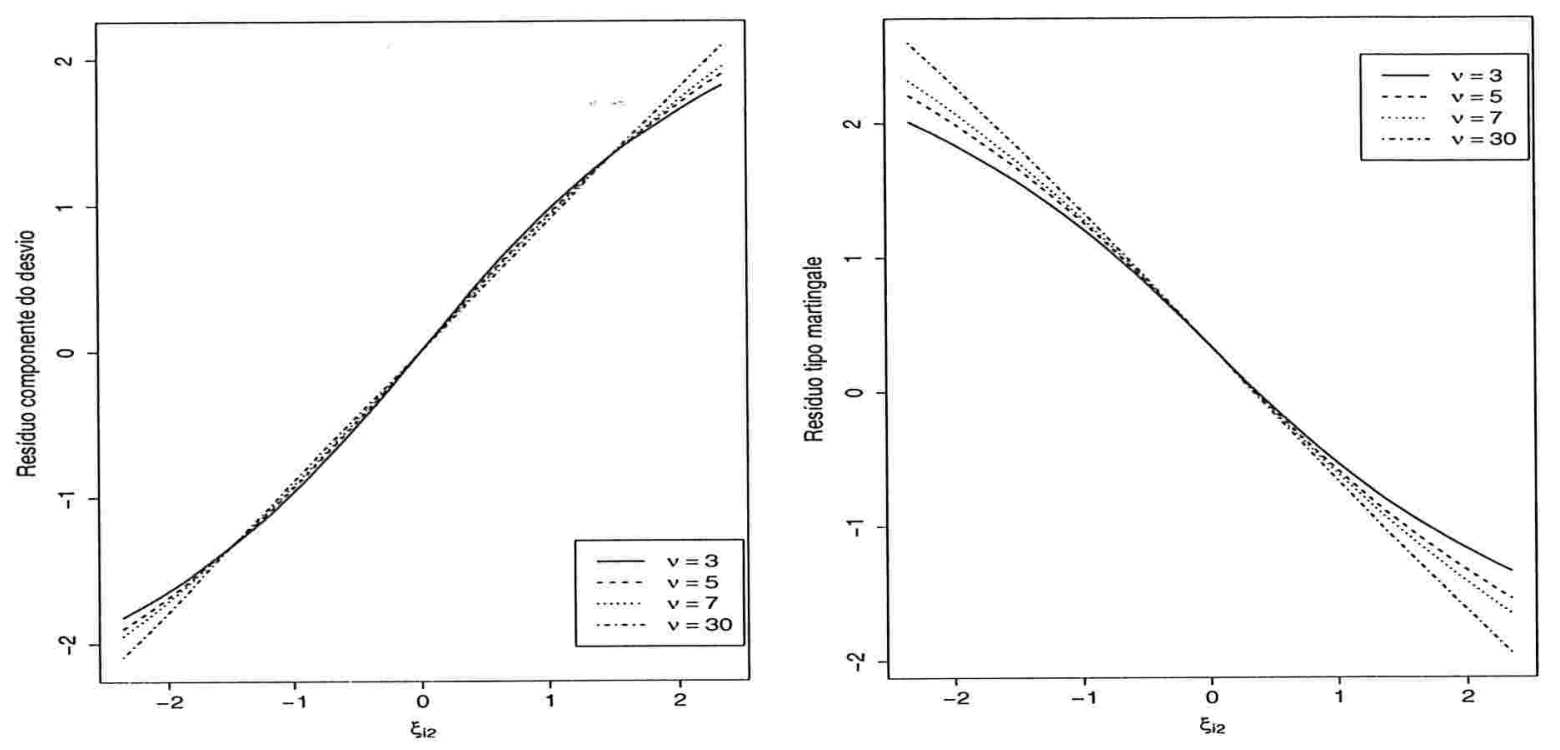

Figura 4.3: comportamento dos resíduos $\mathrm{r}_{D C_{i}}$ (a) e $\mathrm{r}_{M D_{i}}$ (b) contra o resíduo senh para observações não censuradas.

\subsection{Influência local}

As matrizes para o estudo de influência local são obtidas de forma similar ao que foi descrito na Seção 2.2. Portanto, apresentaremos aqui apenas a matriz $\Delta$ para cada esquema de perturbação considerado.

\section{Ponderação de casos}

A matriz $\Delta$ assume a forma dada em (2.12), $\Delta_{\beta}=\boldsymbol{X}^{\top} \operatorname{diag}\left\{\widehat{b}_{1}, \ldots, \widehat{b}_{n}\right\}$ com 


$$
\widehat{b}_{i}= \begin{cases}\frac{1}{2}\left(\widehat{\xi}_{i 1} \widehat{\xi}_{i 2} w\left(\hat{\xi}_{i 2}^{2}\right)-\widehat{\xi}_{i 2} / \widehat{\xi}_{i 1}\right), & \text { se } i \in D \\ \frac{\widehat{\xi}_{i 1}}{2} h\left(\widehat{\xi}_{i 2}\right), & \text { se } i \in C\end{cases}
$$

e $\Delta_{\alpha}=\left(\widehat{a}_{1}, \ldots, \widehat{a}_{n}\right) \mathrm{com}$

$$
\widehat{a}_{i}= \begin{cases}\frac{1}{\hat{\alpha}}\left[\hat{\xi}_{i 2}^{2} w\left(\hat{\xi}_{i 2}^{2}\right)-1\right], & \text { se } i \in D \\ \frac{\hat{\xi}_{i 2}}{\widehat{\alpha}} h\left(\widehat{\xi}_{i 2}\right), & \text { se } i \in C\end{cases}
$$

em que $w(\cdot)$ e $h(\cdot)$ são dados em (4.8), e $\widehat{\xi}_{i 1}$ e $\widehat{\xi}_{i 2}$ são as estimativas de máxima verossimilhança de $\xi_{i 1}$ e $\xi_{i 2}$, respectivamente, dadas em (2.2).

\section{Perturbação da variável resposta}

Neste caso, $\Delta_{\beta}=\boldsymbol{X}^{\top} \operatorname{diag}\left\{\widehat{d}_{1}, \ldots, \widehat{d}_{n}\right\}$, em que

$\widehat{d}_{i}= \begin{cases}S_{y}\left[\frac{1}{\widehat{\alpha}^{2}} \cosh \left(y_{i}-\widehat{\mu}_{i}\right) w\left(\hat{\xi}_{i 2}^{2}\right)-\frac{1}{4} \operatorname{sech}^{2}\left(\frac{y_{i}-\widehat{\mu}_{i}}{2}\right)-\frac{2}{\widehat{\alpha}^{4}} \operatorname{senh}\left(y_{i}-\widehat{\mu}_{i}\right) w^{\prime}\left(\hat{\xi}_{i 2}^{2}\right)\right], & \text { se } i \in D ; \\ \frac{S_{y}}{4}\left[\widehat{\xi}_{i 2} h\left(\widehat{\xi}_{i 2}\right)+\hat{\xi}_{i 1}^{2} h^{\prime}\left(\widehat{\xi}_{i 2}\right)\right], & \text { se } i \in C ;\end{cases}$

e $\Delta_{\alpha}=\left(\widehat{c}_{1}, \ldots, \widehat{c}_{n}\right)$, com

$$
\widehat{c}_{i}= \begin{cases}\frac{S_{y}}{\widehat{\alpha}}\left[\widehat{\xi}_{i 1} \widehat{\xi}_{i 2} w\left(\hat{\xi}_{i 2}^{2}\right)+\hat{\xi}_{i 1} \hat{\xi}_{i 2}^{3} w^{\prime}\left(\hat{\xi}_{i 2}^{2}\right)\right], & \text { se } i \in D \\ \frac{S_{y}}{2 \widehat{\alpha}}\left[\widehat{\xi}_{i 1} h\left(\widehat{\xi}_{i 2}\right)+\widehat{\xi}_{i 1} \widehat{\xi}_{i 2} h^{\prime}\left(\widehat{\xi}_{i 2}\right)\right], & \text { se } i \in C\end{cases}
$$

em que $w(\cdot)$ e $h(\cdot)$ estão definidos em (4.8), $w^{\prime}(\cdot)$ e $h^{\prime}(\cdot)$ são suas derivadas e $\widehat{\xi}_{i 1}$ e $\widehat{\xi}_{i 2}$ são as estimativas de máxima verossimilhança de $\xi_{i 1}$ e $\xi_{i 2}$, respectivamente, dadas em (2.2).

\section{Perturbação em uma variável explicativa}

Aqui $\Delta_{\beta}$ é uma matriz $p \times n$ com elementos $\Delta_{\beta_{i j}}$, para $j \neq t$, dados por

$$
\Delta_{\beta_{i j}}= \begin{cases}S_{x} \widehat{\beta}_{t} x_{i j}\left[\frac{1}{4} \operatorname{sech}^{2}\left(\frac{y_{i}-\widehat{\mu}_{i}}{2}\right)-\frac{1}{\widehat{\alpha}^{2}} \cosh \left(y_{i}-\widehat{\mu}_{i}\right) w\left(\hat{\xi}_{i 2}^{2}\right)\right. & \\ \left.-\frac{2}{\widehat{\alpha}^{2}} \operatorname{senh}^{2}\left(y_{i}-\widehat{\mu}_{i}\right) w^{\prime}\left(\hat{\xi}_{i 2}^{2}\right)\right], & \text { se } i \in D \\ -\frac{S_{x} \widehat{\beta}_{t} x_{i j}}{4}\left[\widehat{\xi}_{i 2} h\left(\widehat{\xi}_{i 2}\right)+\left(\widehat{\xi}_{i 1}\right)^{2} h^{\prime}\left(\widehat{\xi}_{i 2}\right)\right], & \text { se } i \in C\end{cases}
$$


ao passo que para $j=t$ temos

$$
\Delta_{\beta_{i t}}= \begin{cases}S_{x} \widehat{\beta}_{t} x_{i t}\left[\frac{1}{4} \operatorname{sech}^{2}\left(\frac{y_{i}-\widehat{\mu}_{i}}{2}\right)-\frac{1}{\widehat{\alpha}^{2}} \cosh \left(y_{i}-\widehat{\mu}_{i}\right) w\left(\hat{\xi}_{i 2}^{2}\right)-\frac{2}{\widehat{\alpha}^{2}} \operatorname{senh}^{2}\left(y_{i}-\widehat{\mu}_{i}\right) w^{\prime}\left(\hat{\xi}_{i 2}^{2}\right)\right] & \\ +S_{x}\left[\frac{1}{\widehat{\alpha}^{2}} \operatorname{senh}\left(y_{i}-\widehat{\mu}_{i}\right) w\left(\hat{\xi}_{i 2}^{2}\right)-\frac{1}{2} \tanh \left(\frac{y_{i}-\widehat{\mu}_{i}}{2}\right)\right], & \text { se } i \in D \\ -\frac{S_{x} \widehat{\beta}_{t} x_{i t}}{4}\left[\widehat{\xi}_{i 2} h\left(\widehat{\xi}_{i 2}\right)+\left(\widehat{\xi}_{i 1}\right)^{2} h^{\prime}\left(\widehat{\xi}_{i 2}\right)\right]+\frac{S_{x}}{2} \widehat{\xi}_{i 1} h\left(\widehat{\xi}_{i 2}\right), & \text { se } i \in C\end{cases}
$$

e $\Delta_{\alpha}=\left(\widehat{\phi}_{1}, \ldots, \widehat{\phi}_{n}\right)$, com

$$
\widehat{\phi}_{i}= \begin{cases}-\frac{2}{\hat{\alpha}^{3}} S_{x} \widehat{\beta}_{t} \operatorname{senh}\left(y_{i}-\widehat{\mu}_{i}\right)\left[w\left(\hat{\xi}_{i 2}^{2}\right)+\hat{\xi}_{i 2}^{2} w^{\prime}\left(\hat{\xi}_{i 2}^{2}\right)\right], & \text { se } i \in D ; \\ -\frac{\widehat{\beta}_{t} S_{x}}{2 \widehat{\alpha}}\left[\widehat{\xi}_{i 1} h\left(\widehat{\xi}_{i 2}\right)+\frac{2}{\widehat{\alpha}^{2}} \operatorname{senh}\left(y_{i}-\widehat{\mu}_{i}\right) h^{\prime}\left(\widehat{\xi}_{i 2}\right)\right], & \text { se } i \in C,\end{cases}
$$

em que $w(\cdot)$ e $h(\cdot)$ estão definidos em $(4.8), w^{\prime}(\cdot)$ e $h^{\prime}(\cdot)$ são suas derivadas e $\widehat{\xi}_{i 1}$ e $\widehat{\xi}_{i 2}$ são as estimativas de máxima verossimilhança de $\xi_{i 1}$ e $\xi_{i 2}$, respectivamente, dadas em (2.2).

É válido ressaltar que os resultados apresentados neste capítulo são extensões daqueles apresentados no Capítulo 2 e, portanto, considerando $g(u)=e^{-u / 2}$ e $c=\frac{1}{\sqrt{2 \pi}}$, reduzimos o modelo log-BSG ao modelo log-BS. Adicionalmente, em Barros, Paula e Leiva (2006), podem ser encontradas expressões particularizadas para o modelo $t$ de Student log-BS.

\subsection{Aplicação}

\section{Estudo dos tempos de sobrevivência de pacientes com câncer de pulmão}

O conjunto de dados sobre o estudo dos tempos de sobrevivência de pacientes com câncer de pulmão, analisado no Capítulo 2 sob o modelo de regressão linear log-BS, será analisado novamente aqui sob a perspectiva do modelo de regressão linear $t$ de Student log-BS, expresso por

$$
\log \left(t_{i}\right)=\beta_{0}+\beta_{1} x_{i 1}+\cdots+\beta_{8} x_{i 8}+\varepsilon_{i}, i=1, \ldots, 137,
$$

em que $\varepsilon_{i}$ são variáveis aleatórias mutuamente independentes tais que $\varepsilon_{i} \sim \log -\operatorname{BSG}\left(\alpha, 0 ; t_{3}\right)$ com os graus de liberdade sendo escolhidos pela maximização do logaritmo da função de verossimilhança para vários valores de $\nu$. As estimativas de máxima verossimilhança para $\boldsymbol{\theta}=\left(\boldsymbol{\beta}^{\top}, \alpha\right)^{\top}=\left(\beta_{0}, \beta_{1}, \ldots, \beta_{8}, \alpha\right)^{\top}$ (erro padrão aproximado entre parênteses) são

$$
\widehat{\beta}_{0}=2,065(0,636), \widehat{\beta}_{1}=0,036(0,004), \widehat{\beta}_{2}=0,004(0,010), \widehat{\beta}_{3}=0,008(0,009),
$$




$$
\begin{gathered}
\widehat{\beta}_{4}=-0,010(0,021), \widehat{\beta}_{5}=-0,004(0,267), \widehat{\beta}_{6}=-0,723(0,242), \\
\widehat{\beta}_{7}=-0,746(0,256), \widehat{\beta}_{8}=-0,076(0,176) \text { e } \widehat{\alpha}=0,816(0,073) .
\end{gathered}
$$

Nesta análise, as variáveis $x_{2}, x_{3}, x_{4}, x_{5}$ e $x_{8}$ são marginalmente não significativas a um nível de $5 \%$. Assim, o modelo de regressão linear $t$ de Student log-BS seleciona preliminarmente as variáveis medida de aleatorização do estado do paciente (Karnofsky) e tipo histológico do tumor.

As Figuras 4.4(a) e 4.4(b) apresentam o gráfico dos resíduos componente do desvio contra os valores ajustados e o gráfico normal de probabilidade com envelope gerado, respectivamente. Não notamos nenhuma tendência na Figura 4.4(a), apesar de alguns resíduos negativos grandes em módulo (\#77 e \#85). Da Figura 4.4(b) temos que a suposição de que os erros possuem uma distribuição $t$ de Student log-BS não parece ser inadequada. Nenhuma observação fica fora do envelope.

Contudo, os gráficos de $\left|\ell_{\max }\right|$ para $\mathrm{C}_{\ell}(\boldsymbol{\beta})$ e $\mathrm{C}_{\ell}(\alpha)$ sob o esquema de perturbação da ponderação de casos, apresentados nas Figuras 4.5(a) e 4.5(b), respectivamente, revelam novas observações, a saber: \#12, \#95 e \#106. As observações em destaque nesses gráficos correspondem a pacientes que têm em comum valores altos para o tempo do diagnóstico até a aleatorização. Na Figura 4.6, onde os gráficos de $C_{i}(\boldsymbol{\beta})(4.6(\mathrm{a}))$ e $\mathrm{C}_{i}(\alpha)(4.6(\mathrm{~b}))$ contra o índice das observações sob o esquema de perturbação da ponderação de casos são mostrados, poucas observações se evidenciam.

Assim como a análise feita no Capítulo 2, as quatro observações apontadas nas Figuras 4.5 e 4.6 são excluídas e as mudanças relativas são calculadas, bem como os novos níveis descritivos (ver Tabela 4.2). Apesar de grandes variações serem notadas nas estimativas não significativas, nenhuma mudança inferencial é observada. A Figura 4.7 apresenta as estimativas dos pesos $w\left(\xi_{i 2}^{2}\right)$ contra os resíduos componente do desvio $\mathrm{r}_{D C_{i}}$ e, como era esperado, resíduos grandes, alguns deles detectados na primeira análise (Figura 2.12), recebem pesos menores. Portanto, o modelo de regressão $t$ de Student log-BS parece ser mais apropriado para ajustar esse conjunto de dados. Assim, o modelo selecionado é dado por

$$
\log \left(t_{i}\right)=\beta_{0}+\beta_{1} x_{i 1}+\beta_{5} x_{i 5}+\beta_{6} x_{i 6}+\beta_{7} x_{i 7}+\varepsilon_{i}, i=1, \ldots, 137,
$$

com as estimativas de máxima verossimilhança (erro padrão aproximado entre parênteses)

$$
\begin{gathered}
\widehat{\beta}_{0}=2,462(0,336), \widehat{\beta}_{1}=0,036(0,004), \widehat{\beta}_{5}=0,048(0,260), \\
\widehat{\beta}_{6}=-0,664(0,234), \widehat{\beta}_{7}=-0,738(0,253) \text { e } \widehat{\alpha}=0,817(0,073),
\end{gathered}
$$

interpretadas como segue. O tempo de sobrevivência é esperado crescer com a medida de aleatorização do estado do paciente (Karnofsky) e não há diferença significativa entre os tipos 


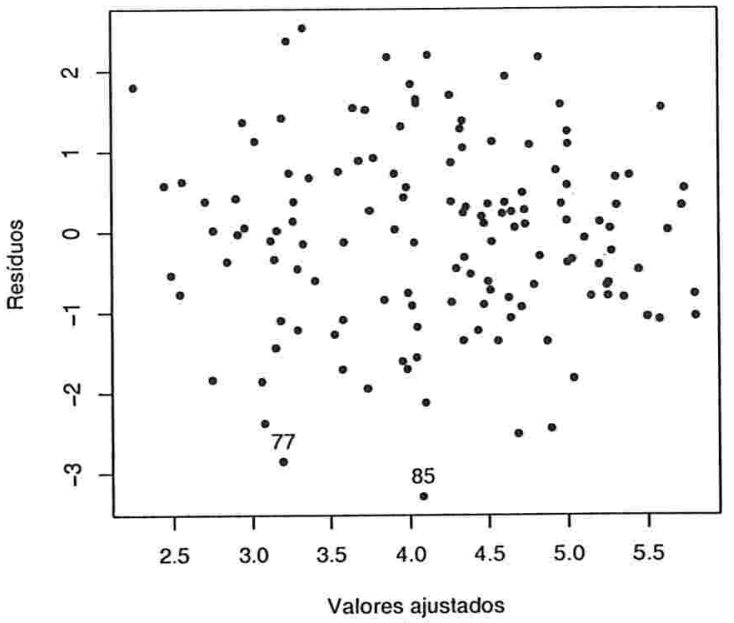

(a)

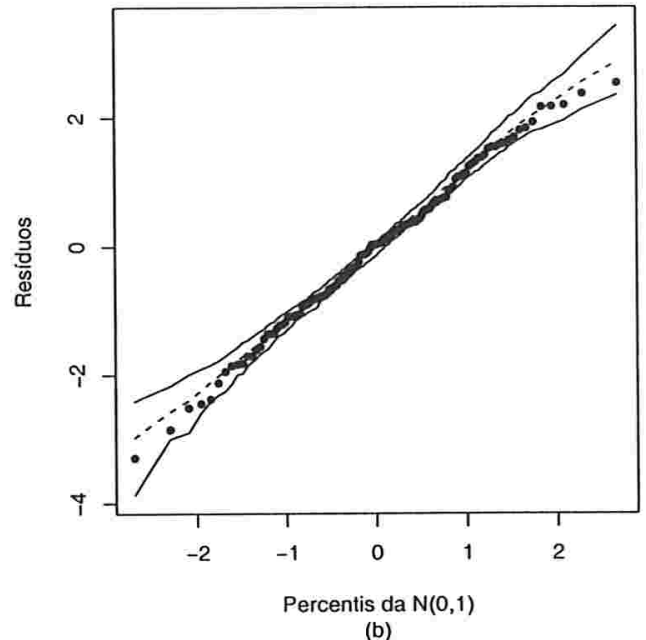

(b)

Figura 4.4: gráfico dos resíduos componente do desvio contra os valores ajustados (a) e gráfico normal de probabilidade dos resíduos componente do desvio com envelope gerado (b).

de tumor squamous e large, mas o tempo de sobrevivência esperado deverá decrescer $1,94\left[e^{0,664}\right]$ e $2,09\left[e^{0,738}\right]$, respectivamente, para os tipos de tumor small e adeno com respeito ao large, assumindo que a medida de aleatorização do estado do paciente (Karnofsky) está fixada. 

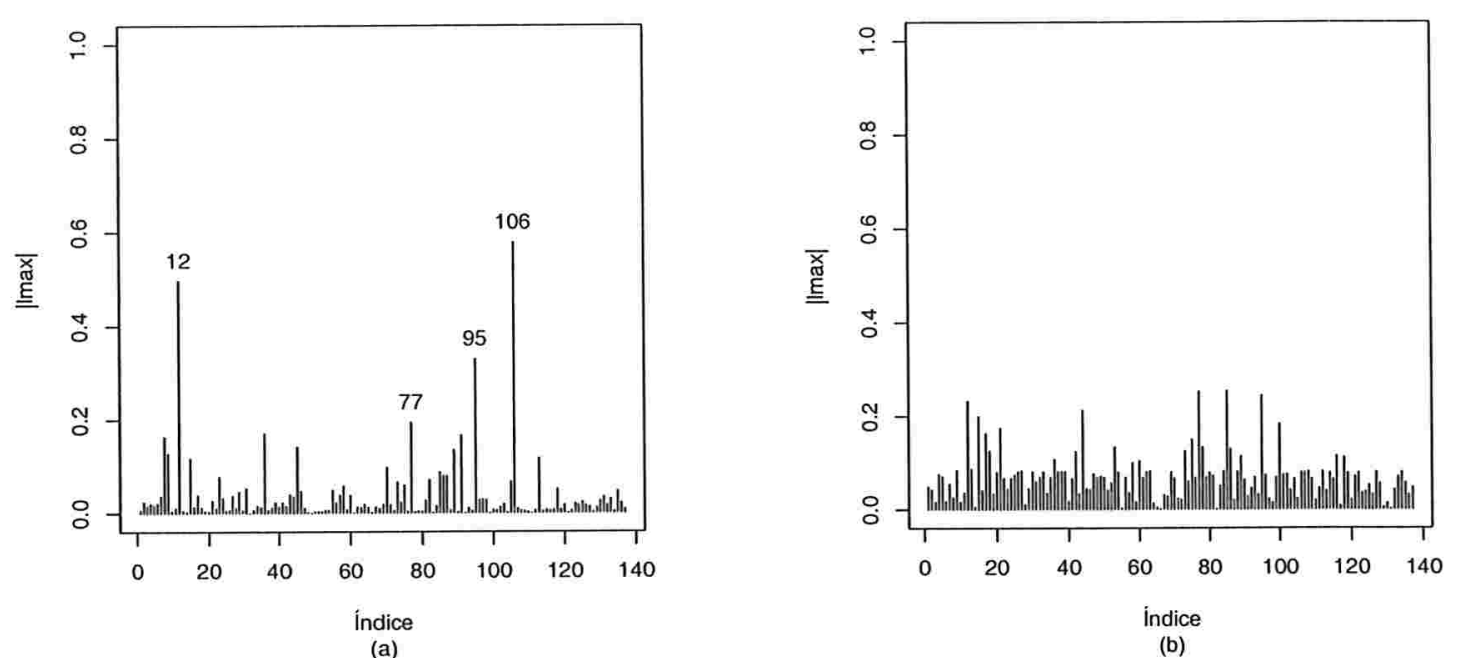

Figura 4.5: gráficos de $\left|l_{\max }\right|$ contra o índice das observações sob o esquema de perturbação da ponderação de casos: (a) interesse em $\boldsymbol{\beta}$, (b) interesse em $\alpha$.

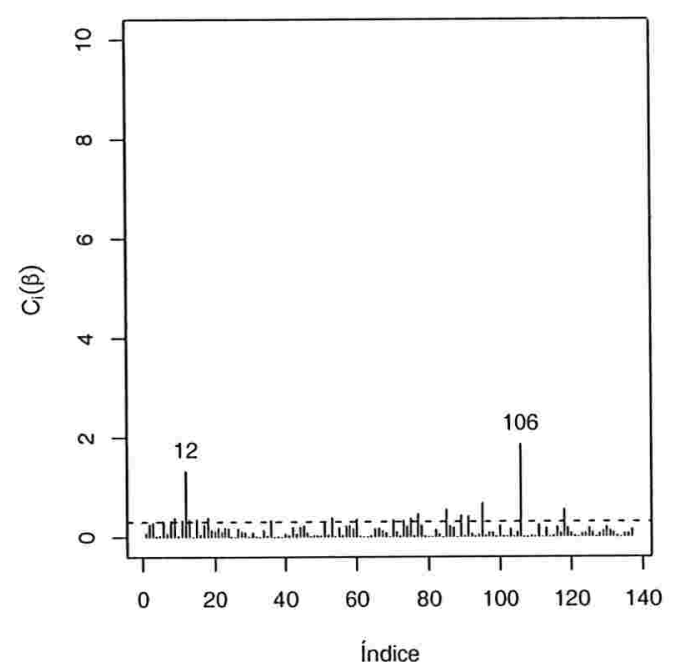

(a)

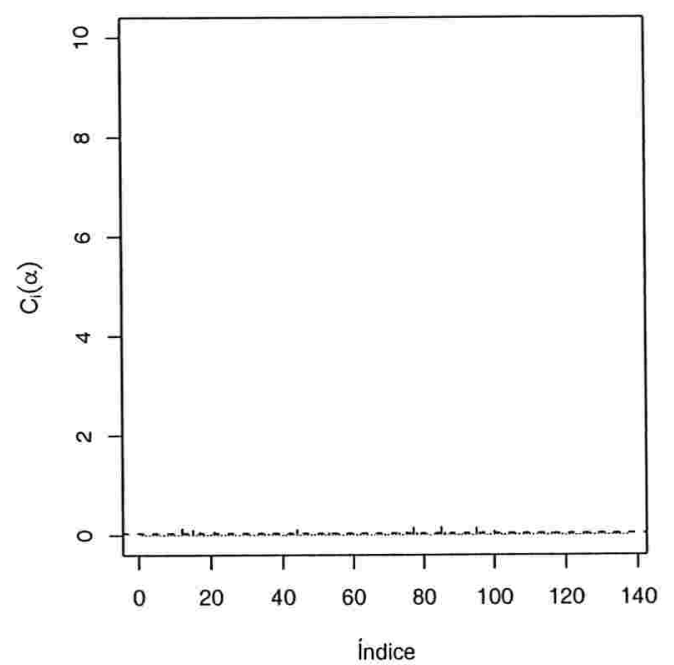

(b)

Figura 4.6: gráficos de $C_{i}(\beta)$ (a) e $C_{i}(\alpha)$ (b) contra o índice das observações sob o esquema de perturbação da ponderação de casos. 


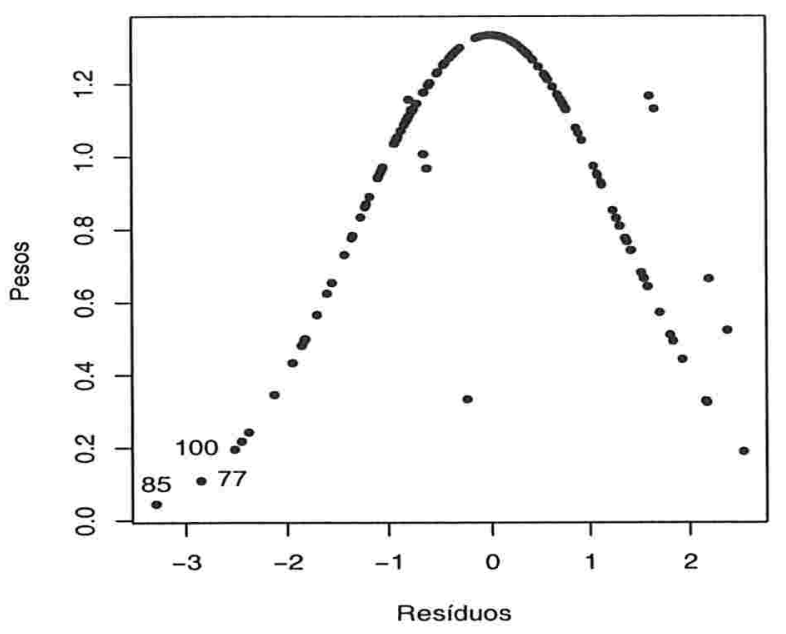

Figura 4.7: pesos estimados $\left(w\left(\widehat{\xi}_{i 2}^{2}\right)\right)$ sob o modelo de regressão linear $t$ de Student log-BS contra os resíduos componente do desvio.

Tabela 4.2: mudanças relativas $(\mathrm{RC}$, em \%) e os correspondentes níveis descritivos entre parênteses.

\begin{tabular}{ccccccc}
\hline & & \multicolumn{5}{c}{ Observação eliminada } \\
Estimativas dos coeficientes & Nenhuma & 12 & 77 & 95 & 106 & Conjunto I \\
\hline$\widehat{\beta}_{0}$ & - & 3 & -1 & -4 & -8 & -5 \\
& $(0.001)$ & $(0.001)$ & $(0.001)$ & $(0.001)$ & $(0.001)$ & $(0.001)$ \\
$\widehat{\beta}_{1}$ & - & 1 & 3 & 0 & 1 & 4 \\
& $(0.000)$ & $(0.000)$ & $(0.000)$ & $(0.000)$ & $(0.000)$ & $(0.000)$ \\
$\widehat{\beta}_{2}$ & - & -171 & -23 & -89 & 471 & -113 \\
& $(0.730)$ & $(0.274)$ & $(0.657)$ & $(0.463)$ & $(0.318)$ & $(0.569)$ \\
$\widehat{\beta}_{3}$ & - & -10 & -3 & 25 & 2 & 13 \\
& $(0.372)$ & $(0.318)$ & $(0.353)$ & $(0.497)$ & $(0.384)$ & $(0.422)$ \\
$\widehat{\beta}_{4}$ & - & -7 & 31 & 23 & 42 & 60 \\
& $(0.634)$ & $(0.604)$ & $(0.740)$ & $(0.710)$ & $(0.787)$ & $(0.843)$ \\
$\widehat{\beta}_{5}$ & - & 1018 & 1216 & 87 & 1146 & 2642 \\
& $(0.988)$ & $(0.882)$ & $(0.857)$ & $(0.998)$ & $(0.868)$ & $(0.681)$ \\
$\widehat{\beta}_{6}$ & - & -2 & -1 & 6 & -7 & 1 \\
& $(0.003)$ & $(0.002)$ & $(0.002)$ & $(0.004)$ & $(0.002)$ & $(0.002)$ \\
$\widehat{\beta}_{7}$ & - & 2 & 0 & 1 & -3 & 3 \\
& $(0.004)$ & $(0.004)$ & $(0.003)$ & $(0.003)$ & $(0.003)$ & $(0.003)$ \\
$\widehat{\beta}_{8}$ & - & -46 & 21 & 46 & -47 & 6 \\
& $(0.669)$ & $(0.527)$ & $(0.732)$ & $(0.813)$ & $(0.533)$ & $(0.677)$ \\
$\widehat{\alpha}$ & - & 2 & 2 & 2 & 0 & 6 \\
\hline
\end{tabular}




\section{Capítulo 5}

\section{Considerações Finais}

Neste trabalho estudamos alguns aspectos da análise de diagnóstico e também de inferência em modelos de regressão log-Birnbaum-Saunders para observações censuradas, considerando censura aleatória. Em particular, derivamos curvaturas normais da influência local através da medida afastamento pela verossimilhança para três esquemas de perturbação e apresentamos algumas formas de se conduzir uma análise residual. Propomos dois tipos de resíduos, cuja distribuição empírica sugere uma boa concordância com a distribuição normal padrão. A metodologia utilizada foi ilustrada através de dados reais da área médica, mostrando que a análise de diagnóstico desempenha um papel importante na seleção do modelo apropriado.

Um outro aspecto abordado foi o estudo da nova classe de distribuições de sobrevivência, a distribuição Birnbaum-Saunders generalizada. Derivamos os momentos da distribuição sob algumas distribuições elípticas geradoras da distribuição Birnbaum-Saunders generalizada e discutimos alguns métodos de estimação dos parâmetros da distribuição.

Mostramos, também, que as estimativas de máxima verossimilhança do modelo logBirnbaum-Saunders clássico são vulneráveis a observações extremas, podendo mascarar a significância dos coeficientes de regressão. Diante disso, propomos uma nova classe de modelos de regressão, os modelos de regressão log-Birnbaum-Saunders generalizados, que reúnem distribuições com caudas mais pesadas do que a distribuição log-Birnbaum-Saunders clássica, o que pode atenuar a influência de tais observações. Em particular, discutimos o modelo $t$ de Student log-Birnbaum-Saunders. A vantagem desse modelo é que é possível manter o método de máxima verossimilhança e obter estimativas robustas na presença de observações extremas no sentido dos resíduos componente do desvio e tipo martingale. 


\subsection{Trabalhos futuros}

Como possíveis trabalhos futuros propomos, inicialmente, considerar o modelo BirnbaumSaunders generalizado com uma função de ligação e preditor linear, isto é, supondo que $T_{i} \sim B S G\left(\alpha, \theta_{i} ; g\right)$ com $h\left(\theta_{i}\right)=\eta_{i}, \eta_{i}=\beta_{1} x_{1 i}+\beta_{2} x_{2 i}+\ldots+\beta_{p} x_{p i}$. Também, podemos estender os métodos de diagnóstico para a classe de modelos de regressão Birnbaum-Saunders heteroscedásticos, em que $\alpha_{i}=m(\gamma), \gamma=\left(\gamma_{1}, \ldots, \gamma_{g}\right)$.

Um outro aspecto que pode ser desenvolvido é o estudo de testes para avaliar a qualidade do ajuste em modelos de regressão Birnbaum-Saunders. Recentemente, Zhu e Zang (2004) desenvolveram um teste baseado em influência local que fornece um diagnóstico global da qualidade do ajuste e que pode ser aplicado a diversas classes de modelos de regressão. A adaptação deste teste aos modelos de regressão Birnbaum-Saunders pode ser investigada. 


\section{Apêndice A}

\section{Expressões do Capítulo 2}

\section{A.1 Funções escore}

A função escore para $\alpha$ é dada por

$$
U_{\alpha}(\theta)=\frac{\partial l(\theta)}{\partial \alpha}=\sum_{i \in D}\left\{\frac{1}{\xi_{i 1}} \frac{\partial \xi_{i 1}}{\partial \alpha}-\frac{1}{2}\left(2 \xi_{i 2} \frac{\partial \xi_{i 2}}{\partial \alpha}\right)\right\}+\sum_{i \in C}\left(-\frac{\phi\left(\xi_{i 2}\right)}{1-\Phi\left(\xi_{i 2}\right)}\right) \frac{\partial \xi_{i 2}}{\partial \alpha} .
$$

Temos que

$$
\begin{gathered}
\frac{\partial \xi_{i 1}}{\partial \alpha}=-\frac{2}{\alpha^{2}} \cosh \left(\frac{y_{i}-\mu_{i}}{2}\right)=-\frac{\xi_{i 1}}{\alpha}, \\
\frac{\partial \xi_{i 2}}{\partial \alpha}=-\frac{2}{\alpha^{2}} \operatorname{senh}\left(\frac{y_{i}-\mu_{i}}{2}\right)=-\frac{\xi_{i 2}}{\alpha}, \\
\frac{\partial \xi_{i 1}}{\partial \beta_{j}}=\frac{2}{\alpha} \operatorname{senh}\left(\frac{y_{i}-\mu_{i}}{2}\right)\left(-\frac{x_{i j}}{2}\right)=-\frac{x_{i j} \xi_{i 2}}{2}, \\
\frac{\partial \xi_{i 2}}{\partial \beta_{j}}=\frac{2}{\alpha} \cosh \left(\frac{y_{i}-\mu_{i}}{2}\right)\left(-\frac{x_{i j}}{2}\right)=-\frac{x_{i j} \xi_{i 1}}{2},
\end{gathered}
$$

$j=1, \ldots, p, i=1, \ldots, n$.

Sustituindo (A.2) e (A.3) em (A.1) obtemos

$$
\begin{aligned}
U_{\alpha}(\theta) & =\sum_{i \in D}\left\{\frac{1}{\xi_{i 1}}\left(-\frac{\xi_{i 1}}{\alpha}\right)-\xi_{i 2}\left(-\frac{\xi_{i 2}}{\alpha}\right)\right\}-\sum_{i \in C}\left(\frac{\phi\left(\xi_{i 2}\right)}{1-\Phi\left(\xi_{i 2}\right)}\right)\left(-\frac{\xi_{i 2}}{\alpha}\right) \\
& =\sum_{i \in D}\left\{-\frac{1}{\alpha}+\frac{\xi_{i 2}^{2}}{\alpha}\right\}+\sum_{i \in C} \xi_{i 2} h\left(\xi_{i 2}\right) \\
& =\frac{1}{\alpha}\left[\sum_{i \in D}\left(\xi_{i 2}^{2}-1\right)+\sum_{i \in C} \xi_{i 2} h\left(\xi_{i 2}\right)\right],
\end{aligned}
$$


em que $h(\cdot)=\frac{\phi(\cdot)}{1-\Phi(\cdot)}$, com $\phi(\cdot)$ denotando a função de densidade de probabilidade da distribuição normal padrão.

A função escore para $\beta_{j}(j=1, \ldots, p)$ é dada por

$$
U_{\beta_{j}}(\theta)=\frac{\partial l(\theta)}{\partial \beta_{j}}=\sum_{i \in D}\left\{\frac{1}{\xi_{i 1}} \frac{\partial \xi_{i 1}}{\partial \beta_{j}}-\frac{1}{2}\left(2 \xi_{i 2} \frac{\partial \xi_{i 2}}{\partial \beta_{j}}\right)\right\}+\sum_{i \in C}\left(-\frac{\phi\left(\xi_{i 2}\right)}{1-\Phi\left(\xi_{i 2}\right)}\right) \frac{\partial \xi_{i 2}}{\partial \beta_{j}} .
$$

Sustituindo (A.4) e (A.5) em (A.6) obtemos

$$
\begin{aligned}
U_{\beta_{j}}(\theta) & =\sum_{i \in D}\left\{\frac{1}{\xi_{i 1}}\left(-\frac{x_{i j} \xi_{i 2}}{2}\right)-\xi_{i 2}\left(-\frac{x_{i j} \xi_{i 1}}{2}\right)\right\}-\sum_{i \in C} h\left(\xi_{i 2}\right)\left(-\frac{x_{i j} \xi_{i 1}}{2}\right) \\
& =\sum_{i \in D}\left\{-\frac{x_{i j}}{2}\left(\frac{\xi_{i 2}}{\xi_{i 1}}-\xi_{i 1} \xi_{i 2}\right)\right\}+\frac{1}{2} \sum_{i \in C} x_{i j} \xi_{i 1} h\left(\xi_{i 2}\right),
\end{aligned}
$$

$j=1, \ldots, p$.

Agora, observe que

$$
\frac{\xi_{i 2}}{\xi_{i 1}}=\frac{\frac{2}{\alpha} \operatorname{senh}\left(\frac{y_{i}-\mu_{i}}{2}\right)}{\frac{2}{\alpha} \cosh \left(\frac{y_{i}-\mu_{i}}{2}\right)}=\tanh \left(\frac{y_{i}-\mu_{i}}{2}\right)
$$

e

$$
\begin{aligned}
\xi_{i 1} \xi_{i 2} & =\frac{2}{\alpha} \cosh \left(\frac{y_{i}-\mu_{i}}{2}\right) \frac{2}{\alpha} \operatorname{senh}\left(\frac{y_{i}-\mu_{i}}{2}\right) \\
& =\frac{4}{\alpha^{2}} \cosh \left(\frac{y_{i}-\mu_{i}}{2}\right) \operatorname{senh}\left(\frac{y_{i}-\mu_{i}}{2}\right) \\
& =\frac{2}{\alpha^{2}} \operatorname{senh}\left(y_{i}-\mu_{i}\right),
\end{aligned}
$$

em que a última igualdade decorre do fato de que $\sinh (x) \cosh (x)=\frac{1}{2} \sinh (2 x)$.

Portanto, a partir de (A.8) e (A.9), podemos reescrever (A.7) da seguinte forma:

$$
U_{\beta_{j}}(\theta)=\sum_{i \in D}\left[\frac{x_{i j}}{\alpha^{2}} \operatorname{senh}\left(y_{i}-\mu_{i}\right)-\frac{x_{i j}}{2} \tanh \left(\frac{y_{i}-\mu_{i}}{2}\right)\right]+\frac{1}{2} \sum_{i \in C} x_{i j} \xi_{i 1} h\left(\xi_{i 2}\right)
$$

$j=1, \ldots, p$.

\section{A.2 Matriz hessiana}

Temos que a matriz hessiana é dada por 


$$
\ddot{\boldsymbol{L}}_{\theta \theta}=\left[\begin{array}{ll}
\ddot{\boldsymbol{L}}_{\alpha \alpha} & \ddot{\boldsymbol{L}}_{\alpha \beta} \\
\ddot{\boldsymbol{L}}_{\beta \alpha} & \ddot{\boldsymbol{L}}_{\beta \beta}
\end{array}\right]=\left[\begin{array}{cc}
\frac{\partial^{2} l(\theta)}{\partial \alpha \partial \alpha} & \frac{\partial^{2} l(\theta)}{\partial \alpha \partial \beta} \\
\frac{\partial^{2} l(\theta)}{\partial \beta^{\top} \partial \alpha} & \frac{\partial^{2} l(\theta)}{\partial \beta^{\top} \partial \beta}
\end{array}\right],
$$

em que $l(\theta)$ é o logaritmo da função de verossimilhança dado em (2.2). Segue que

$$
\begin{aligned}
\ddot{\boldsymbol{L}}_{\alpha \alpha} & =\sum_{i \in D}\left\{\frac{1}{\alpha^{2}}-\frac{\xi_{i 2}^{2}}{\alpha^{2}}+\frac{1}{\alpha} 2 \xi_{i 2} \frac{\partial \xi_{i 2}}{\partial \alpha}\right\}+\sum_{i \in C}\left\{-\frac{1}{\alpha^{2}} \xi_{i 2} h\left(\xi_{i 2}\right)+\frac{1}{\alpha}\left[\frac{\partial \xi_{i 2}}{\partial \alpha} h\left(\xi_{i 2}\right)+\xi_{i 2} \frac{\partial h\left(\xi_{i 2}\right)}{\partial \alpha}\right]\right\} \\
& =\sum_{i \in D}\left\{\frac{1}{\alpha^{2}}-\frac{\xi_{i 2}^{2}}{\alpha^{2}}+\frac{1}{\alpha} 2 \xi_{i 2} \frac{\partial \xi_{i 2}}{\partial \alpha}\right\}+\sum_{i \in C}\left\{-\frac{1}{\alpha^{2}} \xi_{i 2} h\left(\xi_{i 2}\right)+\frac{1}{\alpha}\left[\frac{\partial \xi_{i 2}}{\partial \alpha} h\left(\xi_{i 2}\right)+\xi_{i 2} h^{\prime}\left(\xi_{i 2}\right) \frac{\partial \xi_{i 2}}{\partial \alpha}\right]\right\} .
\end{aligned}
$$

De (A.3) temos

$$
\ddot{\boldsymbol{L}}_{\alpha \alpha}=\sum_{i \in D}\left\{\frac{1}{\alpha^{2}}-\frac{3 \xi_{i 2}^{2}}{\alpha^{2}}\right\}-\sum_{i \in C}\left\{\frac{2}{\alpha^{2}} \xi_{i 2} h\left(\xi_{i 2}\right)+\frac{1}{\alpha^{2}} \xi_{i 2}^{2} h^{\prime}\left(\xi_{i 2}\right)\right\},
$$

$\operatorname{com} h^{\prime}\left(\xi_{i 2}\right)=\frac{\phi^{\prime}\left(\xi_{i 2}\right)}{1-\Phi\left(\xi_{i 2}\right)}+\left(\frac{\phi\left(\xi_{i 2}\right)}{1-\Phi\left(\xi_{i 2}\right)}\right)^{2}$.

Em notação matricial,

$$
\ddot{\boldsymbol{L}}_{\alpha \alpha}=\operatorname{tr}(G),
$$

em que $\boldsymbol{G}=\operatorname{diag}\left\{g_{1}(\theta), \ldots, g_{n}(\theta)\right\}$, com

$$
g_{i}(\boldsymbol{\theta})=\left\{\begin{array}{l}
\frac{1}{\alpha^{2}}-\frac{3 \xi_{i 2}^{2}}{\alpha^{2}}, \text { se } i \in D ; \\
-\frac{2}{\alpha^{2}} \xi_{i 2} h\left(\xi_{i 2}\right)-\frac{1}{\alpha^{2}} \xi_{i 2}^{2} h^{\prime}\left(\xi_{i 2}\right), \text { se } i \in C .
\end{array}\right.
$$

A derivada segunda de $l(\theta)$ com respeito a $\alpha$ e $\beta_{j}$ é dada por

$$
\begin{aligned}
\frac{\partial^{2} l(\theta)}{\partial \alpha \partial \beta_{j}} & =\frac{1}{\alpha} \sum_{i \in D}\left\{2 \xi_{i 2} \frac{\partial \xi_{i 2}}{\partial \beta_{j}}\right\}+\frac{1}{\alpha} \sum_{i \in C}\left\{\frac{\partial \xi_{i 2}}{\partial \beta_{j}} h\left(\xi_{i 2}\right)+\xi_{i 2} \frac{\partial h\left(\xi_{i 2}\right)}{\partial \beta_{j}}\right\} \\
& =\frac{1}{\alpha} \sum_{i \in D}\left\{2 \xi_{i 2} \frac{\partial \xi_{i 2}}{\partial \beta_{j}}\right\}+\frac{1}{\alpha} \sum_{i \in C}\left\{\frac{\partial \xi_{i 2}}{\partial \beta_{j}} h\left(\xi_{i 2}\right)+\xi_{i 2} h^{\prime}\left(\xi_{i 2}\right) \frac{\partial \xi_{i 2}}{\partial \beta_{j}}\right\}
\end{aligned}
$$

Substituindo (A.5) em (A.11) e usando (A.9) obtemos

$$
\begin{aligned}
& \frac{\partial^{2} l(\theta)}{\partial \alpha \partial \beta_{j}}=-\frac{2}{\alpha^{3}} \sum_{i \in D} x_{i j} \operatorname{senh}\left(y_{i}-\mu_{i}\right)-\frac{1}{2 \alpha} \sum_{i \in C} x_{i j} \xi_{i 1} h\left(\xi_{i 2}\right)-\frac{1}{\alpha^{3}} \sum_{i \in C} x_{i j} \operatorname{senh}\left(y_{i}-\mu_{i}\right) h^{\prime}\left(\xi_{i 2}\right), \\
& j=1, \ldots, p .
\end{aligned}
$$


Em notação matricial,

$$
\ddot{\boldsymbol{L}}_{\alpha \beta}=\boldsymbol{K}^{\top} \boldsymbol{X}
$$

em que $\boldsymbol{k}=\left(k_{1}(\boldsymbol{\theta}), \ldots, k_{n}(\boldsymbol{\theta})\right)^{\top}$, com

$$
k_{i}(\theta)=\left\{\begin{array}{l}
-\frac{2}{\alpha^{3}} \operatorname{senh}\left(y_{i}-\mu_{i}\right), \text { se } i \in D \\
-\frac{1}{2 \alpha} \xi_{i 1} h\left(\xi_{i 2}\right)-\frac{1}{\alpha^{3}} \operatorname{senh}\left(y_{i}-\mu_{i}\right) h^{\prime}\left(\xi_{i 2}\right), \text { se } i \in C .
\end{array}\right.
$$

A derivada segunda de $l(\theta)$ com respeito a $\beta_{j}$ e $\beta_{l}$ é dada por

$$
\begin{aligned}
\frac{\partial^{2} l(\theta)}{\partial \beta_{j} \partial \beta_{l}} & =\sum_{i \in D}\left\{\frac{x_{i j}}{\alpha^{2}} \cosh \left(y_{i}-\mu_{i}\right)\left(-\frac{\partial \mu_{i}}{\partial \beta_{l}}\right)-\frac{x_{i j}}{2} \operatorname{sech}^{2}\left(\frac{y_{i}-\mu_{i}}{2}\right)\left(-\frac{1}{2} \frac{\partial \mu_{i}}{\partial \beta_{l}}\right)\right\} \\
& +\frac{1}{2} \sum_{i \in C}\left\{x_{i j} \frac{\partial \xi_{i 1}}{\partial \beta_{l}} h\left(\xi_{i 2}\right)+x_{i j} \xi_{i 1} h^{\prime}\left(\xi_{i 2}\right) \frac{\partial \xi_{i 2}}{\partial \beta_{l}}\right\}
\end{aligned}
$$

usando (A.5) e o fato de que $\frac{\partial \mu_{i}}{\partial \beta_{l}}=x_{i l}$ temos

$$
\begin{aligned}
\frac{\partial^{2} l(\theta)}{\partial \beta_{j} \partial \beta_{l}} & =\sum_{i \in D}\left\{x_{i j} x_{i l}\left[\frac{1}{4} \operatorname{sech}^{2}\left(\frac{y_{i}-\mu_{i}}{2}\right)-\frac{1}{\alpha^{2}} \cosh \left(y_{i}-\mu_{i}\right)\right]\right\} \\
& -\frac{1}{4} \sum_{i \in C} x_{i j} x_{i l}\left\{\xi_{i 2} h\left(\xi_{i 2}\right)+\xi_{i 1}^{2} h^{\prime}\left(\xi_{i 2}\right)\right\} .
\end{aligned}
$$

Em notação matricial,

$$
\ddot{\boldsymbol{L}}_{\beta \beta}=\boldsymbol{X}^{\top} \boldsymbol{V} \boldsymbol{X}
$$

em que $\boldsymbol{V}=\operatorname{diag}\left\{v_{1}(\boldsymbol{\theta}), \ldots, v_{n}(\boldsymbol{\theta})\right\}^{\top} \operatorname{com}$

$$
v_{i}(\theta)=\left\{\begin{array}{l}
\frac{1}{4} \operatorname{sech}^{2}\left(\frac{y_{i}-\mu_{i}}{2}\right)-\frac{1}{\alpha^{2}} \cosh \left(y_{i}-\mu_{i}\right), \text { se } i \in D \\
-\frac{1}{4} \xi_{i 2} h\left(\xi_{i 2}\right)-\frac{1}{4} \xi_{i 1}^{2} h^{\prime}\left(\xi_{i 2}\right), \text { se } i \in C .
\end{array}\right.
$$




\section{A.3 Cálculo das curvaturas}

\section{Ponderação de casos}

Temos que

$$
\frac{\partial l(\boldsymbol{\theta} \mid \boldsymbol{\omega})}{\partial \beta_{j}}=\sum_{i \in D} \omega_{i}\left[\frac{1}{\xi_{i 1}} \frac{\partial \xi_{i 1}}{\partial \beta_{j}}-\frac{1}{2}\left(2 \xi_{i 2} \frac{\partial \xi_{i 2}}{\partial \beta_{j}}\right)\right]+\sum_{i \in C} \omega_{i}\left(-\frac{\phi\left(\xi_{i 2}\right)}{1-\Phi\left(\xi_{i 2}\right)}\right) \frac{\partial \xi_{i 2}}{\partial \beta_{j}},
$$

$j=1, \ldots, p$.

De (A.4) e (A.5) obtemos

$$
\frac{\partial l(\boldsymbol{\theta} \mid \boldsymbol{\omega})}{\partial \beta_{j}}=\sum_{i \in D} \omega_{i} \frac{x_{i j}}{2}\left[\xi_{i 1} \xi_{i 2}-\frac{\xi_{i 2}}{\xi_{i 1}}\right]+\sum_{i \in D} \omega_{i} \frac{x_{i j}}{2} \xi_{i 1} h\left(\xi_{i 2}\right) .
$$

Daí segue que

$$
\frac{\partial^{2} l(\boldsymbol{\theta} \mid \boldsymbol{\omega})}{\partial \beta_{j} \partial \omega_{i}}= \begin{cases}\frac{x_{i j}}{2}\left(\xi_{i 1} \xi_{i 2}-\xi_{i 2} / \xi_{i 1}\right), & \text { se } i \in D \\ \frac{x_{i j}}{2} \xi_{i 1} h\left(\xi_{i 2}\right), & \text { se } i \in C\end{cases}
$$

$j=1, \ldots, p, i=1, \ldots, n$.

Por outro lado,

$$
\frac{\partial l(\boldsymbol{\theta} \mid \boldsymbol{\omega})}{\partial \alpha}=\sum_{i \in D} \frac{\omega_{i}}{\alpha}\left(\xi_{i 2}^{2}-1\right)+\sum_{i \in C} \frac{\omega_{i}}{\alpha} \xi_{i 2} h\left(\xi_{i 2}\right) .
$$

Então,

$$
\frac{\partial^{2} l(\theta \mid \omega)}{\partial \alpha \partial \omega_{i}}= \begin{cases}\frac{1}{\alpha}\left(\xi_{i 2}^{2}-1\right), & \text { se } i \in D \\ \frac{\xi_{i 2}}{\alpha} h\left(\xi_{i 2}\right), & \text { se } i \in C\end{cases}
$$

$i=1, \ldots, n$.

\section{Perturbação na variável resposta}

Neste caso,

$$
\begin{aligned}
\frac{\partial l(\boldsymbol{\theta} \mid \boldsymbol{\omega})}{\partial \beta_{j}} & =\sum_{i \in D}\left[\frac{1}{\xi_{i 1 \omega_{1}}} \frac{\partial \xi_{i 1 \omega_{1}}}{\partial \beta_{j}}-\frac{1}{2}\left(2 \xi_{i 2 \omega_{1}} \frac{\partial \xi_{i 2 \omega_{1}}}{\partial \beta_{j}}\right)\right]+\sum_{i \in C}-h\left(\xi_{i 2 \omega_{1}}\right) \frac{\partial \xi_{i 2 \omega_{1}}}{\partial \beta_{j}} \\
& =-\frac{1}{2} \sum_{i \in D} x_{i j} \frac{\xi_{i 2 \omega_{1}}}{\xi_{i 1 \omega_{1}}}+\frac{1}{2} \sum_{i \in D} x_{i j} \xi_{i 1 \omega_{1}} \xi_{i 2 \omega_{1}}+\frac{1}{2} \sum_{i \in C} x_{i j} \xi_{i 1 \omega_{1}} h\left(\xi_{i 2 \omega_{1}}\right) \\
& =-\frac{1}{2} \sum_{i \in D} x_{i j} \tanh \left(\frac{y_{i \omega}-\mu_{i}}{2}\right)+\frac{1}{\alpha^{2}} \sum_{i \in D} x_{i j} \operatorname{senh}\left(y_{i \omega}-\mu_{i}\right)+\frac{1}{2} \sum_{i \in C} x_{i j} \xi_{i 1 \omega_{1}} h\left(\xi_{i 2 \omega_{1}}\right)
\end{aligned}
$$


e

$$
\frac{\partial^{2} l(\theta \mid \omega)}{\partial \beta_{j} \partial \omega_{i}}= \begin{cases}-\frac{x_{i j}}{2} \operatorname{sech}^{2}\left(\frac{y_{i \omega}-\mu_{i}}{2}\right)\left(\frac{1}{2} \frac{\partial y_{i \omega}}{\partial \omega_{i}}\right)+\frac{1}{\alpha^{2}} \cosh \left(y_{i \omega}-\mu_{i}\right) \frac{\partial y_{i \omega}}{\partial \omega_{i}}, & \text { se } i \in D ; \\ \frac{1}{2} \frac{\partial \xi_{i 1 \omega_{1}}}{\partial \omega_{i}} h\left(\xi_{i 2 \omega_{1}}\right)+\frac{1}{2} \xi_{i 1 \omega_{1}} h^{\prime}\left(\xi_{i 2 \omega_{1}}\right) \frac{\partial \xi_{i 2 \omega_{1}}}{\partial \omega_{i}}, & \text { se } i \in C .\end{cases}
$$

Note que

$$
\begin{gathered}
\frac{\partial y_{i \omega}}{\partial \omega_{i}}=S_{y} \\
\frac{\partial \xi_{i 1 \omega_{1}}}{\partial \omega_{i}}=\frac{2}{\alpha} \operatorname{senh}\left(\frac{y_{i \omega}-\mu_{i}}{2}\right) \frac{S_{y}}{2}=\frac{S_{y}}{2} \xi_{i 2 \omega_{1}}
\end{gathered}
$$

e

$$
\frac{\partial \xi_{i 2 \omega_{1}}}{\partial \omega_{i}}=\frac{2}{\alpha} \cosh \left(\frac{y_{i \omega}-\mu_{i}}{2}\right) \frac{S_{y}}{2}=\frac{S_{y}}{2} \xi_{i 1 \omega_{1}} .
$$

Assim,

$$
\frac{\partial^{2} l(\theta \mid \omega)}{\partial \beta_{j} \partial \omega_{i}}= \begin{cases}-\frac{x_{i j}}{4} S_{y} \operatorname{sech}^{2}\left(\frac{y_{i \omega}-\mu_{i}}{2}\right)+\frac{S_{y}}{\alpha^{2}} \cosh \left(y_{i \omega}-\mu_{i}\right), & \text { se } i \in D \\ \frac{S_{y}}{4} \xi_{i 2 \omega_{1}} h\left(\xi_{i 2 \omega_{1}}\right)+\frac{S_{y}}{4} \xi_{i 1 \omega_{1}} h^{\prime}\left(\xi_{i 2 \omega_{1}}\right), & \text { se } i \in C\end{cases}
$$

$j=1, \ldots, p, i=1, \ldots, n$.

Temos que

$$
\begin{aligned}
\frac{\partial l(\boldsymbol{\theta} \mid \boldsymbol{\omega})}{\partial \alpha} & =\sum_{i \in D}\left[\frac{1}{\xi_{i 1 \omega_{1}}} \frac{\partial \xi_{i 1 \omega_{1}}}{\partial \alpha}-\frac{1}{2}\left(2 \xi_{i 2 \omega_{1}} \frac{\partial \xi_{i 2 \omega_{1}}}{\partial \alpha}\right)\right]+\sum_{i \in C}-h\left(\xi_{i 2 \omega_{1}}\right) \frac{\partial \xi_{i 2 \omega_{1}}}{\partial \alpha} \\
& =-\sum_{i \in D} \frac{1}{\alpha}+\frac{1}{\alpha} \sum_{i \in D} \xi_{i 2 \omega_{1}}^{2}+\frac{1}{\alpha} \sum_{i \in C} \xi_{i 2 \omega_{1}} h\left(\xi_{i 2 \omega_{1}}\right)
\end{aligned}
$$

e

$$
\frac{\partial^{2} l(\theta \mid \omega)}{\partial \alpha \partial \omega_{i}}= \begin{cases}\frac{2}{\alpha} \xi_{i 2 \omega_{1}} \frac{\partial \xi_{i 2 \omega_{1}}}{\partial \omega_{i}}, & \text { se } i \in D \\ \frac{1}{\alpha} \frac{\partial \xi_{i 2 \omega_{1}}}{\partial \omega_{i}} h\left(\xi_{i 2 \omega_{1}}\right)+\frac{1}{\alpha} \xi_{i 2 \omega_{1}} h^{\prime}\left(\xi_{i 2 \omega_{1}}\right) \frac{\partial \xi_{i 2 \omega_{1}}}{\partial \omega_{i}}, & \text { se } i \in C .\end{cases}
$$

De (A.13) podemos reescrever (A.14) da maneira a seguir:

$$
\frac{\partial^{2} l(\theta \mid \omega)}{\partial \alpha \partial \omega_{i}}= \begin{cases}\frac{S_{y}}{\alpha} \xi_{i 1 \omega_{1}} \xi_{i 2 \omega_{1}}, & \text { se } i \in D \\ \frac{S_{y}}{2 \alpha} \xi_{i 1 \omega_{1}} h\left(\xi_{i 2 \omega_{1}}\right)+\frac{S_{y}}{2 \alpha} \xi_{i 1 \omega_{1}} \xi_{i 2 \omega_{1}} h^{\prime}\left(\xi_{i 2 \omega_{1}}\right), & \text { se } i \in C,\end{cases}
$$

$i=1, \ldots, n$. 


\section{Perturbação em uma variável explicativa}

Se $j \neq t$ temos que

$$
\frac{\partial l(\boldsymbol{\theta} \mid \boldsymbol{\omega})}{\partial \beta_{j}}=\sum_{i \in D}\left[\frac{1}{\xi_{i 1 \omega_{2}}} \frac{\partial \xi_{i 1 \omega_{2}}}{\partial \beta_{j}}-\frac{1}{2}\left(2 \xi_{i 2 \omega_{2}} \frac{\partial \xi_{i 2 \omega_{2}}}{\partial \beta_{j}}\right)\right]+\sum_{i \in C}-h\left(\xi_{i 2 \omega_{2}}\right) \frac{\partial \xi_{i 2 \omega_{2}}}{\partial \beta_{j}} .
$$

Observe que

$$
\frac{\partial \xi_{i 1 \omega_{2}}}{\partial \beta_{j}}=-\frac{x_{i j}}{2} \xi_{i 2 \omega_{2}}
$$

e

$$
\frac{\partial \xi_{i 2 \omega_{2}}}{\partial \beta_{j}}=-\frac{x_{i j}}{2} \xi_{i 1 \omega_{2}} .
$$

Logo,

$$
\begin{aligned}
\frac{\partial l(\theta \mid \omega)}{\partial \beta_{j}} & =-\frac{1}{2} \sum_{i \in D} x_{i j} \frac{\partial \xi_{i 2 \omega_{2}}}{\partial \xi_{i 1 \omega_{2}}}+\frac{1}{2} \sum_{i \in D} x_{i j} \xi_{i 1 \omega_{2}} \xi_{i 2 \omega_{2}}+\frac{1}{2} \sum_{i \in C} x_{i j} \xi_{i 1 \omega_{2}} h\left(\xi_{i 2 \omega_{2}}\right) \\
& =-\frac{1}{2} \sum_{i \in D} x_{i j} \tanh \left(\frac{y_{i}-\mu_{i}^{*}}{2}\right)+\frac{1}{\alpha^{2}} \sum_{i \in D} x_{i j} \operatorname{senh}\left(y_{i}-\mu_{i}^{*}\right) \\
& +\frac{1}{2} \sum_{i \in C} x_{i j} \xi_{i 1 \omega_{2}} h\left(\xi_{i 2 \omega_{2}}\right),
\end{aligned}
$$

em que $\mu_{i}^{*}=\mu_{i}+\beta_{t} \omega_{i} S_{x}$. E portanto,

$$
\frac{\partial^{2} l(\theta \mid \omega)}{\partial \beta_{j} \partial \omega_{i}}= \begin{cases}\beta_{t} S_{x} x_{i j}\left[\frac{1}{4} \operatorname{sech}^{2}\left(\frac{y_{i}-\mu_{i}^{*}}{2}\right)-\frac{1}{\alpha^{2}} \cosh \left(y_{i}-\mu_{i}^{*}\right)\right], & \text { se } i \in D ; \\ -\frac{\beta_{t} S_{x} x_{i j}}{4}\left[\xi_{i 2 \omega_{2}} h\left(\xi_{i 2 \omega_{2}}\right)+\xi_{i 1 \omega_{2}}^{2} h^{\prime}\left(\xi_{i 2 \omega_{2}}\right)\right], & \text { se } i \in C\end{cases}
$$

Se $j=t$ temos que

$$
\frac{\partial \xi_{i 1 \omega_{2}}}{\partial \beta_{t}}=-\frac{\xi_{i 2 \omega_{2}}}{2}\left(x_{i t}+\omega_{i} S_{x}\right)
$$

e

$$
\frac{\partial \xi_{i 2 \omega_{2}}}{\partial \beta_{t}}=-\frac{\xi_{i 1 \omega_{2}}}{2}\left(x_{i t}+\omega_{i} S_{x}\right) .
$$

Assim

$$
\begin{aligned}
\frac{\partial l(\boldsymbol{\theta} \mid \boldsymbol{\omega})}{\partial \beta_{t}} & =\sum_{i \in D}\left\{\frac{1}{\xi_{i 1 \omega_{2}}}\left[\frac{-\xi_{i 2 \omega_{2}}}{2}\left(x_{i t}+\omega_{i} S_{x}\right)\right]-\xi_{i 2 \omega_{2}}\left[-\frac{\xi_{i 1 \omega_{2}}}{2}\left(x_{i t}+\omega_{i} S_{x}\right)\right]\right\} \\
& -\sum_{i \in C} h\left(\xi_{i 2 \omega_{2}}\right)\left[\frac{\xi_{i 1 \omega_{2}}}{2}\left(x_{i t}+\omega_{i} S_{x}\right)\right] \\
& =-\frac{1}{2} \sum_{i \in D}\left(x_{i t}+\omega_{i} S_{x}\right)\left[\tanh \left(\frac{y_{i}-\mu_{i}^{*}}{2}\right)+\frac{1}{\alpha^{2}} \operatorname{senh}\left(y_{i}-\mu_{i}^{*}\right)\right]+\frac{1}{2} \sum_{i \in C} h\left(\xi_{i 1 \omega_{2}}\right)\left(x_{i t}+\omega_{i} S_{x}\right)
\end{aligned}
$$


e

$$
\frac{\partial^{2} l(\theta \mid \omega)}{\partial \beta_{t} \partial \omega_{i}}= \begin{cases}\beta_{t} S_{x} x_{i t \omega}\left[\frac{1}{4} \operatorname{sech}^{2}\left(\frac{y_{i}-\mu_{i}^{*}}{2}\right)-\frac{1}{\alpha^{2}} \cosh \left(y_{i}-\mu_{i}^{*}\right)\right] & \text { se } i \in D \\ +S_{x}\left[\frac{1}{\alpha^{2}} \operatorname{senh}\left(y_{i}-\mu_{i}^{*}\right)-\frac{1}{2} \tanh \left(\frac{y_{i}-\mu_{i}^{*}}{2}\right)\right], & \text { se } i \in C . \\ -\frac{\beta_{t} S_{x} x_{i t \omega}}{4}\left[\xi_{i 2 \omega_{2}} h\left(\xi_{i 2 \omega_{2}}\right)+\xi_{i 1 \omega_{2}}^{2} h^{\prime}\left(\xi_{i 2 \omega_{2}}\right)\right]+\frac{S_{x}}{2} \xi_{i 1 \omega_{2}} h\left(\xi_{i 2 \omega_{2}}\right), & \end{cases}
$$

Agora,

$$
\begin{aligned}
\frac{\partial l(\boldsymbol{\theta} \mid \omega)}{\partial \alpha} & =\sum_{i \in D}\left[\frac{1}{\xi_{i 1 \omega_{2}}} \frac{\partial \xi_{i 1 \omega_{2}}}{\partial \alpha}-\frac{1}{2}\left(2 \xi_{i 2 \omega_{2}} \frac{\partial \xi_{i 2 \omega_{2}}}{\partial \alpha}\right)\right]+\sum_{i \in C}-h\left(\xi_{i 2 \omega_{2}}\right) \frac{\partial \xi_{i 2 \omega_{2}}}{\partial \alpha} \\
& =-\sum_{i \in D} \frac{1}{\alpha}+\frac{1}{\alpha} \sum_{i \in D} \xi_{i 2 \omega_{2}}^{2}+\frac{1}{\alpha} \sum_{i \in C} \xi_{i 2 \omega_{2}} h\left(\xi_{i 2 \omega_{2}}\right)
\end{aligned}
$$

$\mathrm{e}$

$$
\frac{\partial^{2} l(\boldsymbol{\theta} \mid \omega)}{\partial \alpha \partial \omega_{i}}= \begin{cases}\frac{1}{\alpha} 2 \xi_{i 2 \omega_{2}} \frac{\partial \xi_{i 2 \omega_{2}}}{\partial \omega_{i}}, & \text { se } i \in D \\ \frac{1}{\alpha} \frac{\partial \xi_{i 2 \omega_{2}}}{\partial \omega_{i}} h\left(\xi_{i 2 \omega_{2}}\right)+\frac{1}{\alpha} \xi_{i 2 \omega_{2}} h^{\prime}\left(\xi_{i 2 \omega_{2}}\right) \frac{\partial \xi_{i 2 \omega_{2}}}{\partial \omega_{i}}, & \text { se } i \in C\end{cases}
$$

que pode ser reescrita como

$$
\frac{\partial^{2} l(\boldsymbol{\theta} \mid \boldsymbol{\omega})}{\partial \alpha \partial \omega_{i}}= \begin{cases}-\frac{2 \beta_{t} S_{x}}{\alpha^{3}} \operatorname{senh}\left(\mathrm{y}_{\mathrm{i}}-\mu_{\mathrm{i}}^{*}\right), & \text { se } i \in D ; \\ -\frac{\beta_{t} S_{x}}{2 \alpha}\left[\xi_{i 1 \omega_{2}} h\left(\xi_{i 2 \omega_{2}}\right)+\frac{2}{\alpha^{2}} \operatorname{senh}\left(\mathrm{y}_{\mathrm{i}}-\mu_{\mathrm{i}}^{*}\right) h^{\prime}\left(\xi_{i 2 \omega_{2}}\right)\right], & \text { se } i \in C,\end{cases}
$$

$i=1, \ldots, n$.

\section{A.4 Resíduo componente do desvio}

O resíduo componente do desvio para as observações não censuradas é definido por

$$
r_{D C_{i}}=\operatorname{sinal}\left(y_{i}-\hat{\mu}_{i}\right) \sqrt{2}\left[l_{i}(\widetilde{\theta})-l_{i}(\widehat{\theta})\right]^{\frac{1}{2}},
$$

em que $\widetilde{\boldsymbol{\theta}}$ é o estimador de máxima verossimilhança de $\boldsymbol{\theta}$ sob o modelo saturado (com $n$ parâmetros), $\widehat{\theta}$ é o estimador de máxima verossimilhança de $\theta$ sob o modelo de interesse (com $p$ parâmetros) e sinal $\left(y_{i}-\hat{\mu}_{i}\right)$ significa o sinal de $\left(y_{i}-\hat{\mu}_{i}\right)$.

Considerando apenas os casos não censurados e assumindo $\alpha$ fixo e $\alpha<2$ temos que o logaritmo da função de verossimilhança fica dado por

$$
l(\theta)=-\frac{1}{2} \sum_{i \in D} \log (8 \pi)+\sum_{i \in D} \log \left[\frac{2}{\alpha} \cosh \left(\frac{y_{i}-\mu_{i}}{2}\right)\right]-\frac{2}{\alpha^{2}} \sum_{i \in D} \operatorname{senh}^{2}\left(\frac{y_{i}-\mu_{i}}{2}\right) .
$$

O estimador de máxima verossimilhança de $\mu_{i}$ para o modelo saturado é solução da equação $\frac{\partial l(\theta)}{\partial \mu_{i}}=0$. Segue que

$$
\frac{\partial l(\theta)}{\partial \mu_{i}}=-\frac{1}{2} \tanh \left(\frac{y_{i}-\mu_{i}}{2}\right)+\frac{1}{\alpha^{2}} \operatorname{senh}\left(y_{i}-\mu_{i}\right) .
$$


Mas,

$$
\frac{\partial l(\theta)}{\partial \mu_{i}}=0 \Leftrightarrow \frac{1}{2} \tanh \left(\frac{y_{i}-\mu_{i}}{2}\right)=\frac{1}{\alpha^{2}} \operatorname{senh}\left(y_{i}-\mu_{i}\right) .
$$

Para resolver a equação acima utilizamos o programa Maple. Essa equação possui cinco raízes, mas apenas uma é uma raiz real, a saber:

$$
\tilde{\mu}_{i}=y_{i}
$$

Desta forma,

$$
l_{i}(\widetilde{\boldsymbol{\theta}})=-\frac{1}{2} \log (8 \pi)+\log \left(\frac{2}{\alpha}\right)
$$

e

$$
l_{i}(\widehat{\theta})=-\frac{1}{2} \log (8 \pi)+\log \left(\frac{2}{\alpha}\right)+\log \left[\cosh \left(\frac{y_{i}-\widehat{\mu}_{i}}{2}\right)\right]-\frac{2}{\alpha^{2}} \operatorname{senh}^{2}\left(\frac{y_{i}-\widehat{\mu}_{i}}{2}\right) .
$$

Segue então que

$$
r_{D C_{i}}=\operatorname{sinal}\left(y_{i}-\hat{\mu}_{i}\right) \sqrt{2}\left\{-\log \left[\cosh \left(\frac{y_{i}-\hat{\mu}_{i}}{2}\right)\right]+\frac{2}{\hat{\alpha}^{2}} \operatorname{senh}^{2}\left(\frac{y_{i}-\hat{\mu}_{i}}{2}\right)\right\}^{\frac{1}{2}} .
$$


Apêndice B

Tabelas referentes às simulações do Capítulo 3 


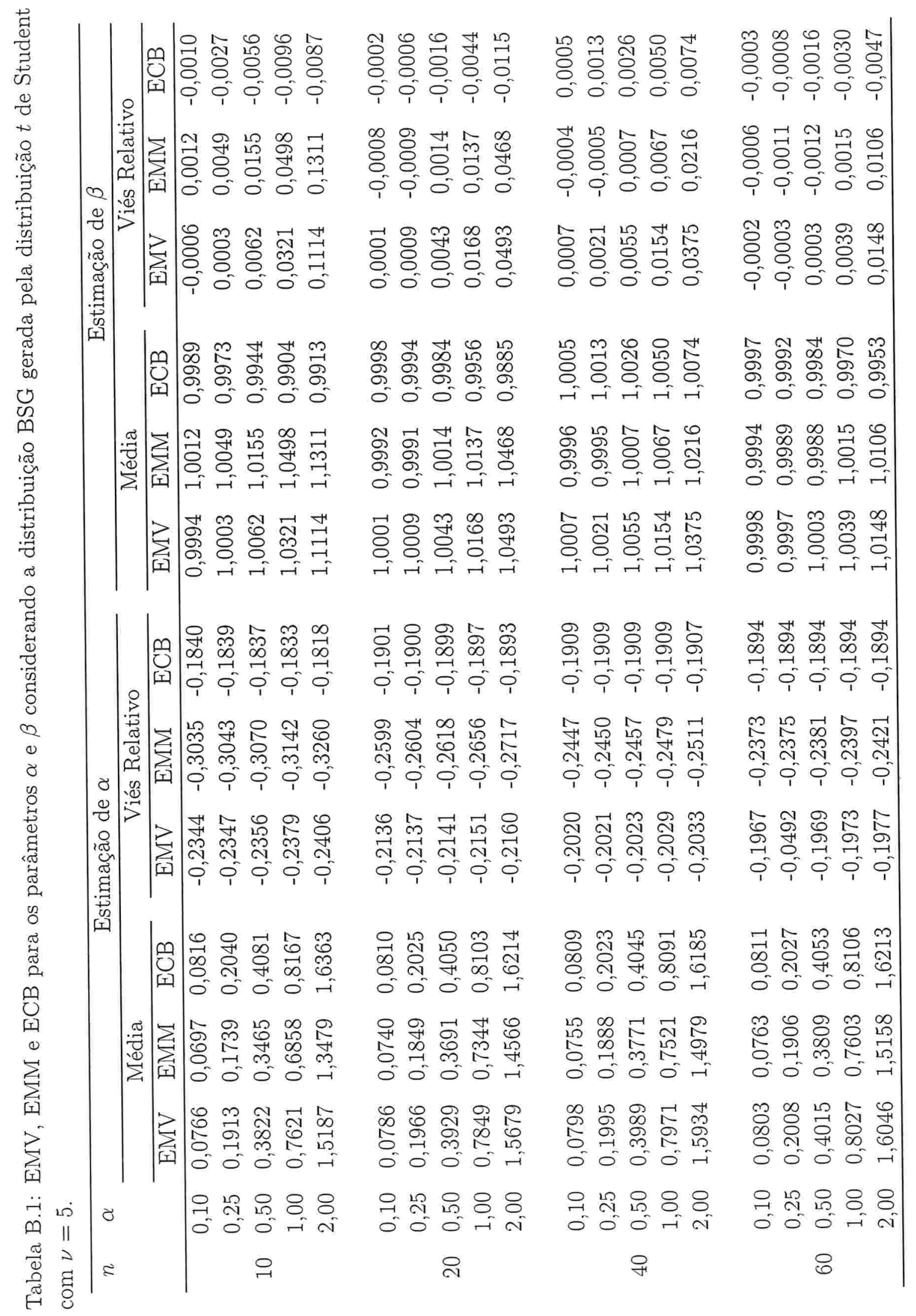




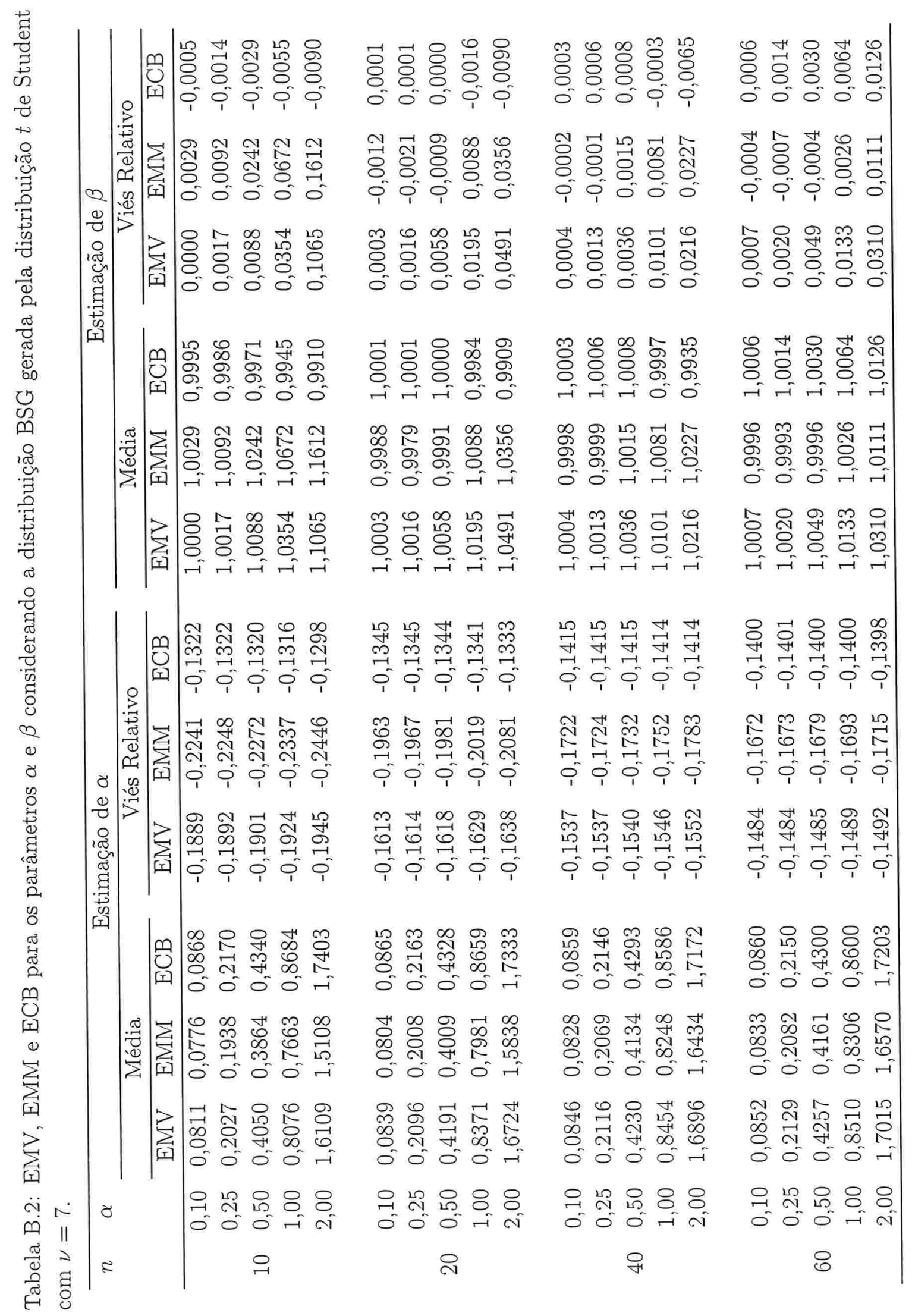

Michelli Barros

IME-USP 


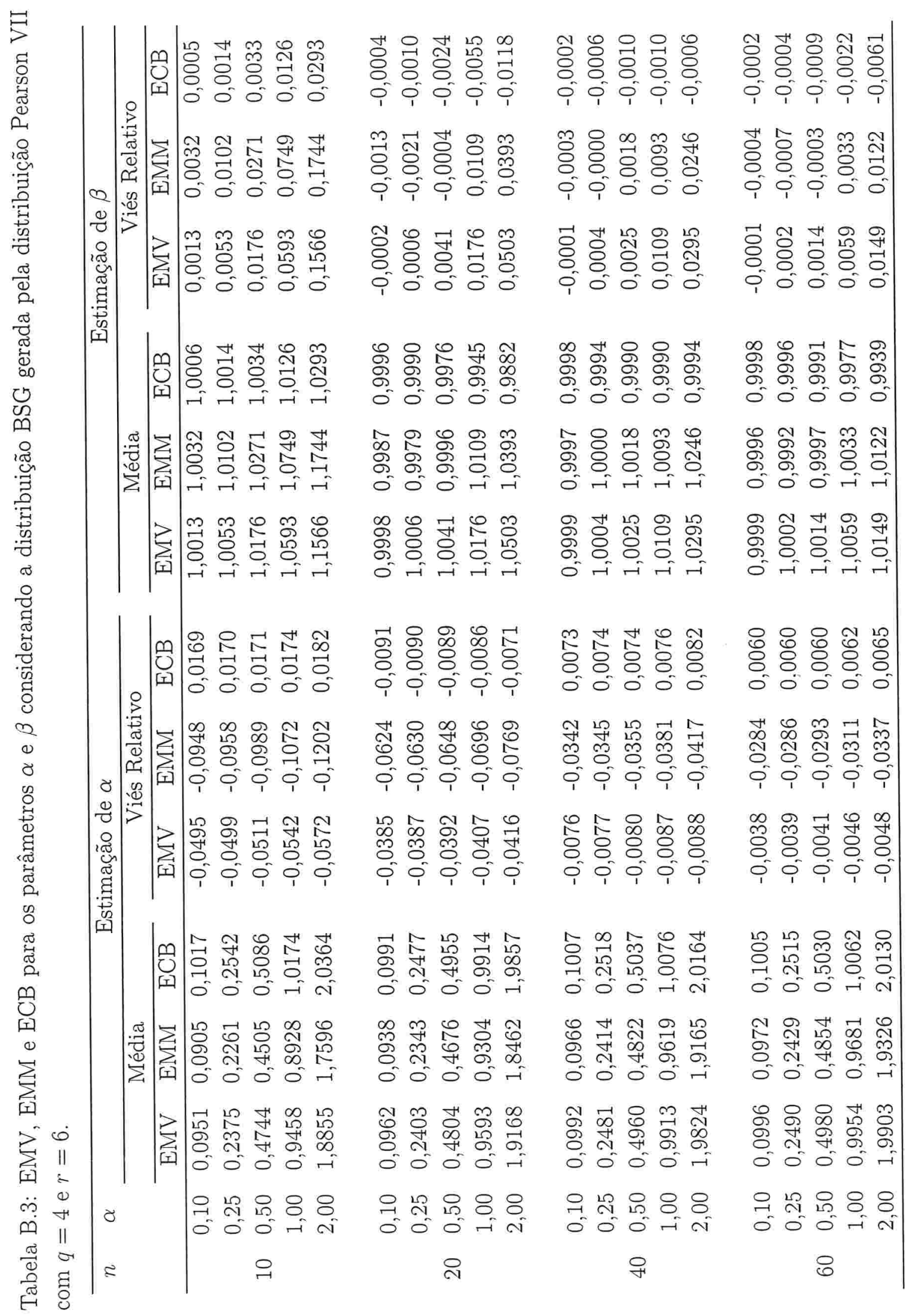




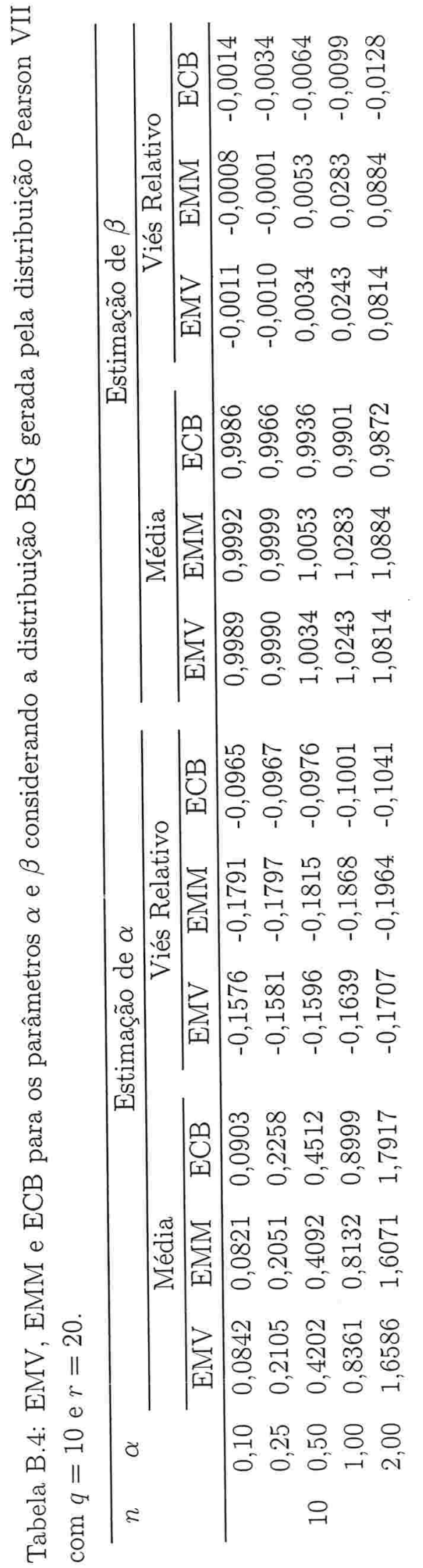

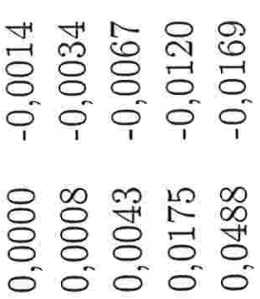

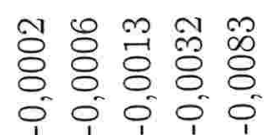

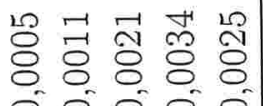
귱융듕 융 용

i 00000

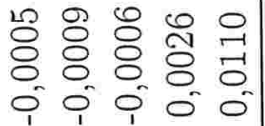

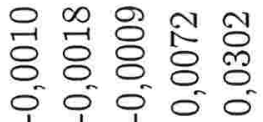

둉ㅎㅇ용용

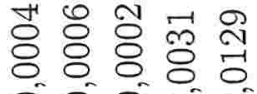
ó

ஜ ஜ ஜ ஜ

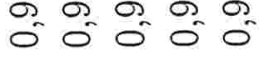

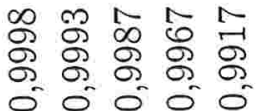

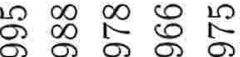

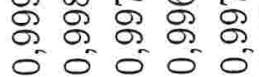
융 ஓ 뒹 등 융 웡

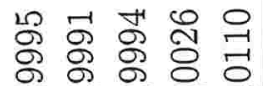
o o o 0

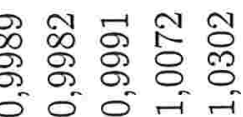

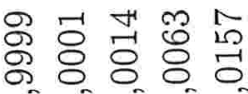

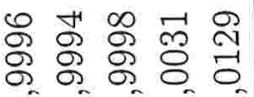

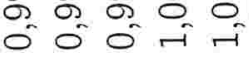

ํํㅇํㅇ융 $\begin{array}{lllll} & & 0 & 0 & 0 \\ 0 & 0 & 0 & 0 \\ 0 & 0 & 0 & 0 & 0 \\ 1 & 1 & 1 & 1 & 1\end{array}$

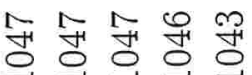
o.

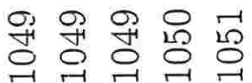

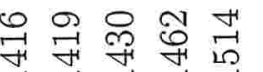

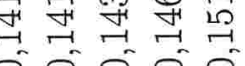

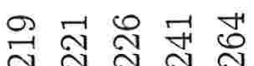
í 긍 그의 ํㅗㅇㅇㅛ 유 극 금ำ 승용ㄷㅇ용드 $\begin{array}{llll}1 & 0 & 0 & 0 \\ 1 & 1 & 1\end{array}$ 年 ô 용 \& ô

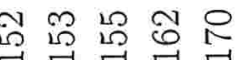
극ㄱㄱ 요요류

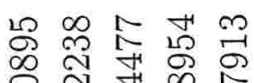
0 o 00 L2 0 o 00 o

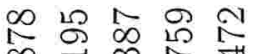

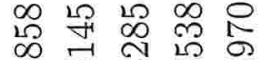
药

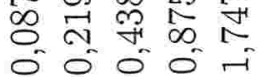
$\begin{array}{lllll}0 & 0 & 0 & -1\end{array}$

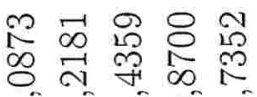

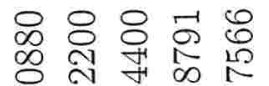
ำ 요 瓷 0000 o o o o ri

బ)

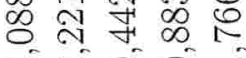
으는는 88 으농요용

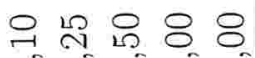
ón 유 아 8 
Tabela B.5: erro quadrático médio das estimativas dos parâmetros $\alpha$ e $\beta$ considerando a distribuição BSG gerada pela distribuição $t$ de Student $\operatorname{com} \nu=5$.

\begin{tabular}{|c|c|c|c|c|c|c|c|}
\hline \multirow[t]{2}{*}{$n$} & \multirow[t]{2}{*}{$\alpha$} & \multicolumn{3}{|c|}{ Estimação de $\alpha$} & \multicolumn{3}{|c|}{ Estimação de $\beta$} \\
\hline & & EMV & EMM & ECB & EMV & EMM & ECB \\
\hline \multirow{5}{*}{10} & 0,10 & 0,0010 & 0,0014 & 0,0009 & 0,0009 & 0,0010 & 0,0009 \\
\hline & 0,25 & 0,0065 & 0,0085 & 0,0058 & 0,0057 & 0,0063 & 0,0058 \\
\hline & 0,50 & 0,0263 & 0,0341 & 0,0231 & 0,0228 & 0,0256 & 0,0226 \\
\hline & 1,00 & 0,1065 & 0,1394 & 0,0931 & 0,0911 & 0,1015 & 0,0857 \\
\hline & 2,00 & 0,4389 & 0,5846 & 0,3809 & 0,3421 & 0,3473 & 0,2982 \\
\hline \multirow{5}{*}{20} & 0,10 & 0,0007 & 0,0009 & 0,0006 & 0,0004 & 0,0005 & 0,0004 \\
\hline & 0,25 & 0,0045 & 0,0056 & 0,0040 & 0,0026 & 0,0032 & 0,0027 \\
\hline & 0,50 & 0,0179 & 0,0227 & 0,0160 & 0,0105 & 0,0128 & 0,0105 \\
\hline & 1,00 & 0,0722 & 0,0925 & 0,0642 & 0,0404 & 0,0479 & 0,0390 \\
\hline & 2,00 & 0,2923 & 0,3826 & 0,2585 & 0,1292 & 0,1425 & 0,1169 \\
\hline \multirow{5}{*}{40} & 0,10 & 0,0005 & 0,0007 & 0,0005 & 0,0002 & 0,0003 & 0,0002 \\
\hline & 0,25 & 0,0033 & 0,0044 & 0,0031 & 0,0014 & 0,0016 & 0,0014 \\
\hline & 0,50 & 0,0132 & 0,0178 & 0,0122 & 0,0054 & 0,0064 & 0,0055 \\
\hline & 1,00 & 0,0529 & 0,0721 & 0,0488 & 0,0206 & 0,0236 & 0,0203 \\
\hline & 2,00 & 0,2128 & 0,2944 & 0,1953 & 0,0605 & 0,0636 & 0,059 \\
\hline \multirow{5}{*}{60} & 0,10 & 0,0005 & 0,0006 & 0,0004 & 0,0001 & 0,0002 & 0,0001 \\
\hline & 0,25 & 0,0029 & 0,0040 & 0,0028 & 0,0009 & 0,0011 & 0,0009 \\
\hline & 0,50 & 0,0119 & 0,0162 & 0,0112 & 0,0036 & 0,0042 & 0,0036 \\
\hline & 1,00 & 0,0476 & 0,0654 & 0,0448 & 0,0136 & 0,0154 & 0,0134 \\
\hline & 2,00 & 0,1913 & 0,2662 & 0,1796 & 0,0407 & 0,0406 & 0,0392 \\
\hline
\end{tabular}


Tabela B.6: erro quadrático médio das estimativas dos parâmetros $\alpha$ e $\beta$ considerando a distribuição BSG gerada pela distribuição $t$ de Student $\operatorname{com} \nu=7$.

\begin{tabular}{|c|c|c|c|c|c|c|c|}
\hline \multirow[t]{2}{*}{$n$} & \multirow[t]{2}{*}{$\alpha$} & \multicolumn{3}{|c|}{ Estimação de $\alpha$} & \multicolumn{3}{|c|}{ Estimação de $\beta$} \\
\hline & & EMV & EMM & ECB & EMV & EMM & ECB \\
\hline \multirow{5}{*}{10} & 0,10 & 0,0008 & 0,0009 & 0,0007 & 0,0009 & 0,0010 & 0,0010 \\
\hline & 0,25 & 0,0052 & 0,0058 & 0,0046 & 0,0059 & 0,0060 & 0,0060 \\
\hline & 0,50 & 0,0211 & 0,0232 & 0,0184 & 0,0234 & 0,0246 & 0,0235 \\
\hline & 1,00 & 0,0854 & 0,0950 & 0,0745 & 0,0921 & 0,0985 & 0,0871 \\
\hline & 2,00 & 0,3535 & 0,4000 & 0,3074 & 0,3228 & 0,3373 & 0,2799 \\
\hline \multirow{5}{*}{20} & 0,10 & 0,0005 & 0,0006 & 0,0005 & 0,0005 & 0,0005 & 0,0005 \\
\hline & 0,25 & 0,0032 & 0,0039 & 0,0029 & 0,0028 & 0,0032 & 0,0029 \\
\hline & 0,50 & 0,0130 & 0,0157 & 0,0115 & 0,0110 & 0,0126 & 0,0110 \\
\hline & 1,00 & 0,0522 & 0,0640 & 0,0459 & 0,0406 & 0,0452 & 0,0390 \\
\hline & 2,00 & 0,2115 & 0,2665 & 0,1848 & 0,1170 & 0,1258 & 0,1053 \\
\hline \multirow{5}{*}{40} & 0,10 & 0,0004 & 0,0004 & 0,0003 & 0,0002 & 0,0003 & 0,0002 \\
\hline & 0,25 & 0,0022 & 0,0027 & 0,0020 & 0,0014 & 0,0016 & 0,0014 \\
\hline & 0,50 & 0,0090 & 0,0107 & 0,0082 & 0,0053 & 0,0062 & 0,0053 \\
\hline & 1,00 & 0,0360 & 0,0434 & 0,0327 & 0,0197 & 0,0223 & 0,0191 \\
\hline & 2,00 & 0,1453 & 0,1782 & 0,1309 & 0,0556 & 0,0588 & 0,0527 \\
\hline \multirow{5}{*}{60} & 0,10 & 0,0003 & 0,0004 & 0,0003 & 0,0002 & 0,0002 & 0,0002 \\
\hline & 0,25 & 0,0019 & 0,0023 & 0,0017 & 0,0010 & 0,0011 & 0,0010 \\
\hline & 0,50 & 0,0075 & 0,0092 & 0,0070 & 0,0039 & 0,0042 & 0,0039 \\
\hline & 1,00 & 0,0302 & 0,0372 & 0,0278 & 0,0143 & 0,0149 & 0,0141 \\
\hline & 2,00 & 0,1215 & 0,1518 & 0,1116 & 0,0403 & 0,0381 & 0,0383 \\
\hline
\end{tabular}


Tabela B.7: erro quadrático médio das estimativas dos parâmetros $\alpha$ e $\beta$ considerando a distribuição BSG gerada pela distribuição Pearson VII com $q=4$ e $r=6$.

\begin{tabular}{|c|c|c|c|c|c|c|c|}
\hline \multirow[t]{2}{*}{$n$} & \multirow[t]{2}{*}{$\alpha$} & \multicolumn{3}{|c|}{ Estimação de $\alpha$} & \multicolumn{3}{|c|}{ Estimação de $\beta$} \\
\hline & & EMV & EMM & ECB & EMV & EMM & ECB \\
\hline \multirow{5}{*}{10} & 0,10 & 0,0007 & 0,0007 & 0,0008 & 0,0011 & 0,0011 & 0,0011 \\
\hline & 0,25 & 0,0043 & 0,0041 & 0,0048 & 0,0067 & 0,0071 & 0,0067 \\
\hline & 0,50 & 0,0171 & 0,0165 & 0,0194 & 0,0274 & 0,0288 & 0,0263 \\
\hline & 1,00 & 0,0688 & 0,0663 & 0,0786 & 0,1099 & 0,1143 & 0,0977 \\
\hline & 2,00 & 0,2852 & 0,2769 & 0,3236 & 0,3659 & 0,3794 & 0,2925 \\
\hline \multirow{5}{*}{20} & 0,10 & 0,0003 & 0,0004 & 0,0003 & 0,0005 & 0,0006 & 0,0005 \\
\hline & 0,25 & 0,0021 & 0,0023 & 0,0022 & 0,0034 & 0,0038 & 0,0034 \\
\hline & 0,50 & 0,0085 & 0,0090 & 0,0087 & 0,0134 & 0,0146 & 0,0132 \\
\hline & 1,00 & 0,0340 & 0,0365 & 0,0351 & 0,0505 & 0,0514 & 0,0481 \\
\hline & 2,00 & 0,1388 & 0,1509 & 0,1437 & 0,1520 & 0,1375 & 0,1340 \\
\hline \multirow{5}{*}{40} & 0,10 & 0,0002 & 0,0002 & 0,0002 & 0,0003 & 0,0003 & 0,0003 \\
\hline & 0,25 & 0,0011 & 0,0012 & 0,0011 & 0,0017 & 0,0018 & 0,0017 \\
\hline & 0,50 & 0,0044 & 0,0047 & 0,0046 & 0,0067 & 0,0072 & 0,0067 \\
\hline & 1,00 & 0,0177 & 0,0188 & 0,0184 & 0,0239 & 0,0254 & 0,0235 \\
\hline & 2,00 & 0,0718 & 0,0765 & 0,0745 & 0,0620 & 0,0637 & 0,0584 \\
\hline \multirow{5}{*}{60} & 0,10 & 0,0001 & 0,0001 & 0,0001 & 0,0002 & 0,0002 & 0,0002 \\
\hline & 0,25 & 0,0007 & 0,0008 & 0,0007 & 0,0011 & 0,0012 & 0,0011 \\
\hline & 0,50 & 0,0029 & 0,0032 & 0,0029 & 0,0044 & 0,0048 & 0,0044 \\
\hline & 1,00 & 0,0115 & 0,0126 & 0,0118 & 0,0157 & 0,0169 & 0,0153 \\
\hline & 2,00 & 0,0462 & 0,0510 & 0,0477 & 0,0395 & 0,0410 & 0,0373 \\
\hline
\end{tabular}


Tabela B.8: erro quadrático médio das estimativas dos parâmetros $\alpha$ e $\beta$ considerando a distribuição BSG gerada pela distribuição Pearson VII com $q=10$ e $r=20$.

\begin{tabular}{|c|c|c|c|c|c|c|c|}
\hline \multirow[t]{2}{*}{$n$} & \multirow[t]{2}{*}{$\alpha$} & \multicolumn{3}{|c|}{ Estimação de $\alpha$} & \multicolumn{3}{|c|}{ Estimação de $\beta$} \\
\hline & & EMV & EMM & ECB & EMV & EMM & ECB \\
\hline \multirow{5}{*}{10} & 0,10 & 0,0007 & 0,0007 & 0,0006 & 0,0009 & 0,0010 & 0,0009 \\
\hline & 0,25 & 0,0041 & 0,0045 & 0,0036 & 0,0057 & 0,0059 & 0,0057 \\
\hline & 0,50 & 0,0165 & 0,0181 & 0,0143 & 0,0226 & 0,0231 & 0,0221 \\
\hline & 1,00 & 0,0674 & 0,0743 & 0,0579 & 0,0869 & 0,0865 & 0,0818 \\
\hline & 2,00 & 0,2829 & 0,3144 & 0,2423 & 0,2922 & 0,2774 & 0,2602 \\
\hline \multirow{5}{*}{20} & 0,10 & 0,0004 & 0,0004 & 0,0003 & 0,0004 & 0,0005 & 0,0004 \\
\hline & 0,25 & 0,0024 & 0,0027 & 0,0020 & 0,0027 & 0,0028 & 0,0027 \\
\hline & 0,50 & 0,0095 & 0,0110 & 0,0081 & 0,0106 & 0,0112 & 0,0106 \\
\hline & 1,00 & 0,0383 & 0,0450 & 0,0327 & 0,0380 & 0,0412 & 0,0368 \\
\hline & 2,00 & 0,1567 & 0,1871 & 0,1326 & 0,1047 & 0,1190 & 0,0960 \\
\hline \multirow{5}{*}{40} & 0,10 & 0,0003 & 0,0003 & 0,0002 & 0,0002 & 0,0002 & 0,0002 \\
\hline & 0,25 & 0,0016 & 0,0017 & 0,0015 & 0,0014 & 0,0015 & 0,0015 \\
\hline & 0,50 & 0,0066 & 0,0067 & 0,0058 & 0,0056 & 0,0057 & 0,0056 \\
\hline & 1,00 & 0,0265 & 0,0270 & 0,0234 & 0,0194 & 0,0201 & 0,0191 \\
\hline & 2,00 & 0,1075 & 0,1102 & 0,0941 & 0,0486 & 0,0515 & 0,0467 \\
\hline \multirow{5}{*}{60} & 0,10 & 0,0002 & 0,0002 & 0,0002 & 0,0002 & 0,0002 & 0,0002 \\
\hline & 0,25 & 0,0013 & 0,0012 & 0,0012 & 0,0010 & 0,0011 & 0,0010 \\
\hline & 0,50 & 0,0052 & 0,0050 & 0,0047 & 0,0038 & 0,0041 & 0,0038 \\
\hline & 1,00 & 0,0210 & 0,0201 & 0,0188 & 0,0133 & 0,0141 & 0,0132 \\
\hline & 2,00 & 0,0853 & 0,0819 & 0,0757 & 0,0347 & 0,0350 & 0,0338 \\
\hline
\end{tabular}




\section{Referências}

Achcar, J. A. e Espinosa, M. M. (1991). Bayesian methodos in accelerated life tests considering a log-linear model for the Birnbaum-Saunders distribution. Revista Brasileira de Estatística 52, 47-68.

Arellano-Valle, R. B. (1994). Distribuições Elípticas: Propriedades, Inferência e Aplicações a Modelos de Regressão. Tese de Doutorado. IME/USP, São Paulo.

Atkinson, A. C. (1981). Two graphical display for outlying and influential observations in regression. Biometrika 68, 13-20.

Atkinson, A. C. (1985). Plots, Transformations and Regression: An Introduction to Graphical Methods of Diagnostic Regression Analysis. Clarendon Press, Oxford.

Barlow, W. E. e Prentice, R. L. (1988). Residuals for relative risk regression. Biometrika 75, 65-74.

Barros, M., Paula, G. A. e Leiva, V. (2006). Student-t log-Birnbaum-Saunders models. Submetido.

Belsley, D. A., Kuh, E. e Welsch, R. E. (1980). Regression Diagnostics: Identifying Influential Data and Sources of Collinearity. Jonh Wiley and Sons, New York.

Birnbaum, Z. W. e Saunders, S. C. (1969a). A new family of life distributions. Journal of Applied Probability 6, 319-327.

Birnbaum, Z. W. e Saunders, S. C. (1969b). Estimation for a family of life distributions with applications to fatigue. Journal of Applied Probability 6, 328-347.

Chatterjee, S. e Hadi, A. S. (1988). Sensitivity Analysis in Linear Regression. Jonh Wiley and Sons, New York.

Cook, R. D. (1977). Detection of influential observation in linear regression. Technometrics 19, 15-18. 
Cook, R. D. (1986). Assessment of local influence (with discussion). Journal of the Royal Statistical Society B 48, 133-169.

Cook, R. D., Peña, D. e Weisberg, S. (1988). The likelihood displacement: A unifying principle for influence measures. Communications in Statistics: Theory and Methods 17, 623-640.

Cook, R. D. e Weisberg, S. (1982). Residuals and Influence in Regression. Chapman and Hall, London.

Cordeiro, G. M. e Paula, G. A. (1992). Estimation, large-sample parametric tests and diagnostics for non-exponential family nonlinear models. Communications in Statistics: Simulation and Computation 21, 149-172.

Cysneiros, F. J. A. (2004). Modelos Restritos e Validação de Modelos Simétricos de Regressão. Tese de Doutorado. IME/USP, São Paulo.

Davison, A. C. e Gigli, A. (1989). Deviance residuals and normal scores plots. Biometrika 76, 211-221.

Davison, A. C. e Tsai, C. L. (1992). Regression model diagnostics. International Statistical Review 60, 337-353.

Desmond, A. (1985). Stochastic models of failure in random environments. Canadian Journal of Statistics 13, 171-183.

Desmond, A. (1986). On the relationship between two fatigue-life models. IEEE Transactions on Reliability $35,167-169$.

Díaz-García, J. A. e Domínguez-Molina, J. R. (2006). Some generalisations of BirnbaumSaunders and sinh-normal distributions. International Mathematical Forum 1, 17091727.

Díaz-García, J. A., Galea, M. e Leiva, V. (2003). Influence diagnostics for elliptical multivariate linear regression models. Communications in Statistics: Theory and Methods 32, 625-641.

Díaz-García, J. A. e Leiva, V. (2005). A new family of life distributions based on the contoured elliptically distributions. Journal of Statistical Planning and Inference 128, 88-95. 
Díaz-García, J. A. e Leiva, V. (2006). Erratum to a new family of life distributions based on the elliptically contoured distributions [Journal of Statistical Planning and Inference 128(2) (2005) 445-457]. To appear in Journal of Statistical Planning and Inference.

Díaz-García, J. A., Leiva, V. e Galea, M. (2002). Singular elliptic distribution: density and applications. Communications in Statistics: Theory and Methods 31, 665-681.

Doornik, J. A. (2001). Ox: an Object-Oriented Matrix Language. fourth ed. Timberlake Consultants Press, London, Oxford. (www.doornik.com).

Dupuis, D. J. e Mills, J. E. (1998). Robust estimation of the Birnbaum-Saunders distribution. IEEE Transactions on Reliability 47, 88-95.

Efron, B. (1979). Bootstrap methods: another look at the jacknife. Annals of Statistics 7, $1-26$.

Efron, B. e Tibishirani, R. J. (1993). An Introduction to the Bootstrap. Chapman and Hall, New York.

Engelhardt, M., Bain, L. J. e Wright, F. T. (1981). Inferences on the parameters of the Birnbaum-Saunders fatigue life distribution based on maximum likelihood estimation. Technometrics 23, 251-255.

Escobar, L. A. e Meeker, W. Q. (1992). Assessing influence in regression analysis with censored data. Biometrics 48, 507-528.

Fang, K. T. e Anderson, T. W. (1990). Statistical Inference in Elliptical Contoured and Related Distributions. Allerton Press, New York.

Fang, K. T., Kotz, S. e Ng, K. W. (1990). Symmetric Multivariate and Related Distributions, Chapman and Hall, London.

Fang, K. T. e Zhang, Y. T. (1990). Generalized Multivariate Analysis. Springer-Verlag, New York.

Fung, W. K. e Kwan, C. W. (1997). A note on local influence based on the normal curvature. Journal of Royal Statistical Society B 59, 839-843.

Galea, M., Leiva, V. e Paula, G. A. (2004). Influence diagnostics in log-Birnbaum-Saunders regression models. Journal of Applied Statistics 31, 1049-1064. 
Galea, M., Paula, G. A. e Bolfarine, H. (1997). Local influence in elliptical linear regression models. The Statistician 46, 71-79.

Galea, M., Riquelme, M. e Paula, G. A., (2000). Diagnostics methods in elliptical linear regression models.Brazilian Journal of Probability and Statistics 14, 167-184.

Ghosh, S. K. e Ghosal, S. (2006). Semiparametric Accelerated Failure Time Models for Censored Data. In: Upadhyay, S.K., Singh, U., Dey, D.K., (Eds.), Bayesian Statistics and its Applications, New Delhi, Anamaya Publishers, 213-229.

Gradshteyn, I. S. e Randzhik, I. M. (2000). Table of Integrals, Series, and Products. Academic Press, New York.

Gupta, A. K. e Varga, T. (1993). Elliptically Contoured Models in Statistics. Kluwer Academic Publishers.

Jin, Z., Lin, D. Y., Wei, L. J. e Ying, Z. (2003). Rank-based inference for the accelerated failure time model. Biometrika 90, 341-353.

Kalbfleisch, J. D. e Prentice, R. L. (2002). The Statistical Analysis of Failure Time Data. John Wiley and Sons, New York.

Klein, J. P. e Moeschberger, M. L. (1997). Survival Analysis: Techniques for Censored and Truncated Data. Springer, New York.

Krall, J. V., Uthoff, V. A. e Harley, J. B. (1975). A step-up procedure for selecting variables associated with survival. Biometrics $31,49-57$.

Kwan, C. W. e Fung, W. K. (1998). Assessing local influence for specific restricted likelihood: Application to factor analysis. Psychometrika 63, 35-46.

Lawless, J. F. (1982). Statistical Models and Methods for Lifetime Data. Wiley, New York.

Lee, E. T. e Wang, J. W. (2003). Statistical Methods for Survival Data Analysis. John Wiley and Sons, New York.

Lee, S. Y., Lu, B. e Song, X. Y. (2006). Assessing local influence for nonlinear structural equation models with ignorable missing data. Computational Statistics and Data Analysis $50,1356-1377$.

Leiva, V., Barros, M., Paula, G. A. e Galea, M. (2006). Influence diagnostics in log-BirnbaumSaunders regression models with censored data. Accepted in Computational Statistics and Data Analysis.

Michelli Barros

IME-USP 
Leiva, V., Barros, M., Paula, G. A. e Sanhueza, A. (2007). Generalized Birnbaum-Saunders Distribution Applied to Air Pollutant Concentration. Accepted in Environmetrics.

Lesaffre, E. e Verbeke, G. (1998). Local influence in linear mixed models. Biometrics 54, 570-582.

Lu, M. "e Chang, D. S. (1997). Bootstrap prediction intervals for the Birnbaum-Saunders distribution. Microelectron Reliability 37, 1213-1216.

McCarter, K. S. (1999). Estimation and Prediction for the Birnbaum-Saunders Distribution Using Type-II Censored Samples, with a Comparison to the Inverse Gaussian Distribution. Ph.D Thesis, Kansas State University, Department of Statistics, College of Arts and Sciences.

McCullagh, P. e Nelder, J. A. (1989). Generalized Linear Models. Chapman and Hall, London.

Moolgavkar, S., Lustbader, E. e Venzon, D. (1984). A geometric approach to nonlinear regression diagnostic with application to matched case-control studies. Annals of Statistics $12,816-826$.

Ng, H. K. T., Kundu, D. e Balakrishnan, N. (2003). Modified moment estimation for the twoparameter Birnbaum-Saunders distribution. Computational Statistics and Data Analysis 43, 283-298.

Ortega, E. M. (2001). Análise de Influência e Resíduos em Modelos de Regressão Log-Gama Generalizados. Tese de Doutorado. IME/USP, São Paulo.

Ortega, E. M., Bolfarine, H. e Paula, G. A. (2003). Influence diagnostics in generalized log-gamma regression models. Computational Statistics and Data Analysis 42, 165-186.

Ospina, R. (2004). Estimação Pontual e Intervalar em um Modelo de Regressão Beta. Dissertação de Mestrado, DE/UFPE, Recife.

Owen, W. J. e Padgett, W. J. (1999). Accelerated test models for system strength based on Birnbaum-Saunders distribution. Lifetime Data Analysis 5, 133-147.

Owen, W. J. e Padgett, W. J. (2000). A Birnbaum-Saunders accelerated life model. IEEE Transactions on Reliability 49, 224-229.

Paula, G. A. (1993). Assessing local influence in restricted regression models. Computational Statistics and Data Analysis 16, 63-79. 
Paula, G. A. e Peres, C. A. (1988). Diagnostics for GLMs with linear inequality parameter constraints. Communications in Statistics: Theory and Methods 17, 4205-4219.

Pettit, A. N. e Bin Daud, I. (1989). Case-weight measures of influence for proportional hazards regression. Applied Statistics 38, 51-67.

Press, W. H., Teulosky, S. A., Vetterling, W, T. e Flannery, B. P. (1992). Numerical Recipes in C: The Art of Scientific Computing. Prentice Hall, London.

Rieck, J. R. (1989). Statistical Analysis for the Birnbaum-Saunders Fatigue Life Distribution. Ph. D. Thesis, Clemson University, Department of Mathematical Sciences.

Rieck, J. R. (1999). A moment-generating function with application to the BirnbaumSaunders distribution. Communications in Statistics: Theory and Methods 28, 22132222 .

Rieck, J. R. e Nedelman, J. R. (1991). A log-linear model for the Birnbaum-Saunders distribution. Technometrics $\mathbf{3 3}, 51-60$.

Saunders, S. C. (1974). A family of random variables closed under reciprocation. Journal of the American Statistical Association 69, 533-539.]

Souza, F. A. M. D. (1999). Influência Local e Análise de Resíduos em Modelos de Regressão Von Mises. Tese de Doutorado. IME/USP, São Paulo.

Tanaka, Y., Zhang, F. e Mori (2003). Local influence in principal component analysis: relationship between the local influence and influence function approaches revisited. Computational Statistics and Data Analysis 44, 143-160.

Therneau, T. M., Grambsch, P. M. e Fleming, T. R. (1990). Martingale-based residuals for survival models. Biometrika 77, 147-160.

Tsionas, E. G. (2001). Bayesian inference in Birnbaum-Saunders regression. Communications in Statistics: Theory and Methods 30, 179-193.

Verbeke, G. e Molenberghs, G. (2000). Linear Mixed Models for Longitudinal Data. Springer, New York.

Vilca-Labra e Leiva, V. (2006). A new fatigue life model based on the family of skew-elliptical distributions. Communications in Statistics: Theory and Methods 35, 1-16. 
Wang, Z., Desmond, A. F., Lu, X. (2006). Modified censored moment estimation for the twoparameter Birnbaum-Saunders distribution. Computational Statistics and Data Analysis 50, 1033-1051.

Wei, B. C., Hu, Y. Q. e Fung, W. K. (1998). Generalized leverage and its applications. Scandinavian Journal of Statistics 25, 25-37.

Williams, D. A. (1987). Generalized linear model diagnostic using the deviance and single case deletion. Applied Statistics 36, 181-191.

Zhu, H. e Zang, H. (2004). A diagnostic procedure based on local influence. Biometrika 91, 579-589. 